CONFORMAL GEOMETRY AND DYNAMICS

An Electronic Journal of the American Mathematical Society

Volume 5, Pages 21-73 (August 8, 2001)

S $1088-4173(01) 00064-9$

\title{
METRIC AND GEOMETRIC QUASICONFORMALITY IN AHLFORS REGULAR LOEWNER SPACES
}

\author{
JEREMY T. TYSON
}

\begin{abstract}
Recent developments in geometry have highlighted the need for abstract formulations of the classical theory of quasiconformal mappings. We modify Pansu's generalized modulus to study quasiconformal geometry in spaces with metric and measure-theoretic properties sufficiently similar to Euclidean space. Our basic objects of study are locally compact metric spaces equipped with a Borel measure which is Ahlfors-David regular of dimension $Q>1$, and satisfies the Loewner condition of Heinonen-Koskela. For homeomorphisms between open sets in two such spaces, we prove the equivalence of three conditions: a version of metric quasiconformality, local quasisymmetry and geometric quasiconformality.

We derive from these results several corollaries. First, we show that the Loewner condition is a quasisymmetric invariant in locally compact Ahlfors regular spaces. Next, we show that a proper $Q$-regular Loewner space, $Q>1$, is not quasiconformally equivalent to any subdomain. (In the Euclidean case, this result is due to Loewner.) Finally, we characterize products of snowflake curves up to quasisymmetric/bi-Lipschitz equivalence: two such products are bi-Lipschitz equivalent if and only if they are isometric and are quasisymmetrically equivalent if and only if they are conformally equivalent.
\end{abstract}

\section{INTRODUCTION}

In recent years, the need for a theory of quasiconformal mappings in general (nonEuclidean) settings has become clear. This point was first observed by Mostow in his pioneering work on the rigidity of hyperbolic space forms [50]. The classical proof of the celebrated Mostow rigidity theorem required the development of an analytic theory of quasiconformal mappings on the boundaries at infinity of the classical rank one symmetric spaces. Quasiconformal maps in the setting of nonabelian Carnot groups were first studied by Mostow and later by Pansu [51], [52. and Korányi and Reimann 42, 43. Until recently, however, it was not entirely clear to what extent the existence of this non-Euclidean theory was dependent on the inherent algebraic structure of these spaces. Heinonen and Koskela [38 have developed an abstract theory of quasiconformal maps on a particular class of metric measure spaces which recovers much of the classical theory in the case of Euclidean spaces or more general Carnot groups. Other examples of spaces satisfying the

Received by the editors May 31, 2000 and, in revised form, June 4, 2001.

2000 Mathematics Subject Classification. Primary 30C65; Secondary 28A78, 46E35, 43A85.

Key words and phrases. Quasiconformal/quasisymmetric map, conformal modulus, Loewner condition, Hausdorff/packing measure, Poincaré inequality.

The results of this paper form part of the author's Ph.D. thesis completed at the University of Michigan in 1999. Research supported by an NSF Graduate Research Fellowship and a Sloan Doctoral Dissertation Fellowship. 
assumptions of the Heinonen-Koskela theory have been constructed by Bourdon and Pajot [9], [10] and Laakso [46]; see section 11] of this paper.

The basic objects of study in the Heinonen-Koskela theory are Ahlfors regular metric measure spaces satisfying the Loewner condition. Recall that a metric space $X=(X, d)$ endowed with a Borel measure $\mu$ is said to be (Ahlfors) $Q$-regular for some $Q \geq 0$ if there exist constants $c_{0}>0$ and $C_{0}<\infty$ so that

$$
c_{0} r^{Q} \leq \mu(B) \leq C_{0} r^{Q}
$$

for every closed ball $B$ in $X$ with radius $r<\operatorname{diam} X$. Such a space necessarily has Hausdorff dimension equal to $Q$. We will describe the Loewner condition for a metric measure space shortly, but in order to motivate its definition we briefly recall some well-known results on the equivalence of various notions of quasiconformality.

Fix $Q \geq 1$ and a pair of $Q$-regular metric measure spaces $(X, d, \mu)$ and $\left(Y, d^{\prime}, \nu\right)$ and let $f: U \rightarrow U^{\prime}$ be a homeomorphism between open sets $U \subset X$ and $U^{\prime} \subset Y$. We say that the map $f$ is metrically $H$-quasiconformal for some $H \geq 1$ if

$$
\limsup _{r \rightarrow 0} \frac{\max \left\{d^{\prime}(f(x), f(y)): d(x, y) \leq r\right\}}{\min \left\{d^{\prime}(f(x), f(z)): d(x, z) \geq r\right\}} \leq H
$$

for each $x \in U$. Furthermore, we say that $f$ is geometrically $K$-quasiconformal for some $K \geq 1$ if

$$
\operatorname{Mod}_{Q} \Gamma / K \leq \operatorname{Mod}_{Q} f \Gamma \leq K \operatorname{Mod}_{Q} \Gamma
$$

for every collection $\Gamma$ of curves lying in the open set $U$. Here $\operatorname{Mod}_{Q} \Gamma \operatorname{denotes}$ the $Q$-modulus of the curve family $\Gamma$, see section [1.

It is well-known (see, for example, Gehring's fundamental paper [29]) that these conditions are quantitatively equivalent for homeomorphisms $f: U \rightarrow U^{\prime}$ between domains in $\mathbb{R}^{n}, n \geq 2$. Indeed, it suffices to require that $(0.3)$ hold only for those families $\Gamma=(E, F)$ which consist of the curves joining a pair of disjoint, nondegenerate continua $E$ and $F$ in $\mathbb{R}^{n}$. The best constants $H$ and $K$ depend only on each other and (possibly) on the dimension $n$.

The proof that $(0.3)$ implies 0.2 in $\mathbb{R}^{n}$ relies on two basic properties of the $n$-modulus: an explicit upper bound for $\operatorname{Mod}_{n}(E, F)$ in the particular case when $E$ and $F$ are the inner and outer boundaries of a spherical shell, and a quantitative lower bound for the $n$-modulus of certain curve families of the type $\Gamma=(E, F)$. The latter estimates are classical when $n=2$; for $n \geq 3$ they were first established in qualitative form by Loewner [4] and later in quantitative form by Gehring [28].

The upper bound for the $Q$-modulus holds more generally whenever the underlying space satisfies the Ahlfors regularity condition (0.1), see (10.6). However, the lower bound reflects deeper properties of Euclidean space. Heinonen and Koskela 38. axiomatize this condition by defining a $Q$-regular metric measure space to be a Loewner space if the corresponding lower bound for the $Q$-modulus holds. (See section 10 for the exact definition.) The Loewner condition can be viewed as a connectivity assumption; roughly speaking, it states that any two pieces of the space can be joined by a "relatively large" family of "short" curves.

The principal message of [38] is that $Q$-regular Loewner spaces, $Q>1$, are a natural setting for quasiconformal geometry. For instance, by [38, Theorem 4.7] metric quasiconformality implies local quasisymmetry for homeomorphisms $f: X \rightarrow Y$ between two such spaces. Recall that $f$ is said to be $\eta$-quasisymmetric (for some 
increasing homeomorphism $\eta:[0, \infty) \rightarrow[0, \infty))$ if

$$
|f x-f y| \leq \eta(t)|f x-f z|
$$

whenever $x, y, z \in X$ and $t>0$ satisfy $|x-y| \leq t|x-z|$.

Our interest in this paper is in the notion of geometric quasiconformality. In 69], the author showed that quasisymmetric homeomorphisms between $Q$-regular spaces, $Q>1$, were geometrically quasiconformal. Note that the Loewner condition is not an a priori requirement for this result, in fact, we may deduce as a corollary that the Loewner condition is a quasisymmetric invariant in this setting (cf. Corollary 12.1). By combining the results of [69] and [38], we see that all three definitions (metric quasiconformality, quasisymmetry, and geometric quasiconformality) are equivalent for homeomorphisms between $Q$-regular Loewner spaces. This class of spaces includes all of the examples mentioned above.

Although already of some importance, the result in [69] is unsatisfying as a complete generalization of Gehring's theorem. The assumptions on the underlying spaces are too strong; both the regularity condition (0.1) and the Loewner condition are global properties which do not hold for arbitrary domains in $\mathbb{R}^{n} \mathbb{1}$ In this paper we modify the arguments we gave in 69] to address this issue. Our results are also motivated by an observation of Heinonen and Koskela [37, Theorem 1.4] that when $X=Y=\mathbb{R}^{n}, n \geq 2$, quasiconformality is already implied by the following weaker metric condition: there exists $H \geq 1$ so that

$$
\liminf _{r \rightarrow 0} \frac{\max \{|f(x)-f(y)|:|x-y|=r\}}{\min \{|f(x)-f(z)|:|x-z|=r\}} \leq H
$$

for each $x \in \mathbb{R}^{n}$. The proof of this fact in [37] relies heavily on the fact that the underlying environment is Euclidean space by making use of certain covering theorems specific to that setting. Heinonen and Koskela ask whether (0.5) also implies quasiconformality in the general setting.

With these motivating remarks in mind, we now state our primary result (cf. Theorem 10.9):

Let $Q>1$ and let $U$ and $U^{\prime}$ be open sets in $Q$-regular Loewner spaces $(X, d, \mu)$ and $\left(Y, d^{\prime}, \nu\right)$, respectively. Then the following conditions are equivalent for a homeomorphism $f: U \rightarrow U^{\prime}$ :

(i) there exist constants $H \geq 1$ and $t>1$ so that

$$
\liminf _{r \rightarrow 0} \frac{\max \left\{d^{\prime}(f(x), f(y)): d(x, y) \leq t r\right\}}{\min \left\{d^{\prime}(f(x), f(z)): d(x, z) \geq r\right\}} \leq H
$$

for all $x \in U$;

(ii) for each $t \geq 1$, there exists $H(t) \geq 1$ so that

$$
\limsup _{r \rightarrow 0} \frac{\max \left\{d^{\prime}(f(x), f(y)): d(x, y) \leq t r\right\}}{\min \left\{d^{\prime}(f(x), f(z)): d(x, z) \geq r\right\}} \leq H(t)
$$

for all $x \in U$;

(iii) there exists $\eta:[0, \infty) \rightarrow[0, \infty)$ so that $f$ is locally $\eta$-quasisymmetric;

(iv) there exists $K \geq 1$ so that $f$ is geometrically $K$-quasiconformal.

\footnotetext{
${ }^{1}$ Both conditions do hold locally in the Euclidean case (for instance, they hold on each Euclidean ball), but it is not at all clear in the abstract setting if the satisfaction of the global regularity or Loewner condition implies the satisfaction of the corresponding local property. Compare however Lemma 10.5
} 
Note here the restriction $t>1$ in (i); our arguments are not strong enough to include the case $t=1$.

In the Euclidean case, the standard proof that metric quasiconformality implies geometric quasiconformality proceeds by first establishing sufficient regularity for the maps (namely, membership in the local Sobolev class $W_{\text {loc }}^{1, n}$ ) and then deriving an analytic version of the quasiconformality condition. Then change of variables in the integrals defining the conformal modulus can be justified by appealing to the absolute continuity of $W^{1, n}$ homeomorphisms in measure (Lusin's condition (N)) and the absolute continuity along $n$-almost every curve (Fuglede's theorem).

Until recently, it appeared as if this technique was too specialized to transfer to the abstract setting of metric spaces. However, Heinonen, Koskela, Shanmugalingam and the author [39] recently established both the Lusin condition $(\mathrm{N})$ and Fuglede's theorem for locally quasisymmetric maps between domains in $Q$-regular Loewner spaces. Thus a proof of our main result can be given along the standard lines and one can even prove the equivalence of definitions (i)-(iv) with an analytic definition of quasiconformality (see Theorem 9.8 of [39]).2 The proof which we present here is independent of [39]. We avoid the analytic issue entirely by relying instead on combinatorial arguments using a generalized definition of the conformal modulus first introduced by Pansu in [51] and developed in [69] 3 Thus even in the Euclidean setting our approach provides a new proof for Gehring's classical theorem.

The organization of this paper is as follows. In section 1 we recall basic notation and definitions. We review in some detail the classical theory of the modulus in the context of general metric measure spaces. This serves as a framework for our discussion of the generalized modulus in section [7 In order to define this latter concept, we first develop a theory of Hausdorff and packing measures relative to general (non gauge-type) set functions which may be of independent interest.

In sections 8 and 9 we prove two crucial properties of the generalized modulus. Roughly speaking, we show that the classical and generalized $Q$-moduli differ by at most a bounded factor in $Q$-regular spaces (section 8) and that the generalized modulus never decreases under homeomorphisms which satisfy (0.6) (section 9). This shows that such mappings satisfy the left-hand inequality in (0.3). In section 10 we define the Loewner property for metric measure spaces and finish the proof of the equivalence of (i)-(iv) under this additional assumption. Section 11]contains brief descriptions of the examples of Bourdon-Pajot and Laakso.

In sections 12 15, we apply our main theorem to a variety of questions regarding quasiconformal maps. In section 12 we first show that the Loewner condition is quasisymmetrically invariant in locally compact Ahlfors $Q$-regular spaces; this answers a conjecture of Heinonen and Koskela from [38. In the Ahlfors $Q$-regular setting, the Loewner condition can be reformulated in terms of an analytic condition (Poincaré inequalities). We mention the role which our results play in the general theory of Poincaré inequalities as a consequence of this fact.

In [47], Loewner proved that the Euclidean space $\mathbb{R}^{n}, n \geq 2$, satisfies a qualitative version of the Loewner condition and used this result to prove that $\mathbb{R}^{n}$ is not

\footnotetext{
${ }^{2}$ Furthermore, Cheeger [19] has defined a notion of "differentiability" for real-valued Lipschitz functions on $Q$-regular Loewner spaces which can be used to construct "differentials" for quasiconformal mappings between two such spaces, cf. $\S 10$ of [39].

${ }^{3}$ For a different use of a notion of "discrete" or "combinatorial" modulus, see the papers of Cannon, Floyd, Perry and Swenson [12, [13, [14].
} 
quasiconformally equivalent to any proper subdomain. In section 13 we prove a version of this result where $\mathbb{R}^{n}$ is replaced by any $Q$-regular Loewner space for which bounded sets are precompact. In section [14 we briefly discuss how our main theorem has been used in [39] to study the aforementioned question of absolute continuity.

Finally, in section 15, we illustrate in the context of a particular example how our main theorem can be used to distinguish at the quasisymmetric or bi-Lipschitz level between homeomorphic metric spaces. For $0<\epsilon \leq 1$, the snowflake curve of order $\epsilon$ is the real line $\mathbb{R}$ endowed with the metric $d_{\epsilon}(x, y)=|x-y|^{\epsilon}$. We prove that the spaces $X=\prod_{i=1}^{n}\left(\mathbb{R}, d_{\epsilon_{i}}\right)$ and $Y=\prod_{i=1}^{n}\left(\mathbb{R}, d_{\delta_{i}}\right)$ are bi-Lipschitz equivalent if and only if the multisets $\left\{\epsilon_{1}, \ldots, \epsilon_{n}\right\}$ and $\left\{\delta_{1}, \ldots, \delta_{n}\right\}$ coincide and that they are quasisymmetrically equivalent if and only if the multisets $\left\{\epsilon_{1}, \ldots, \epsilon_{n}\right\}$ and $\left\{\lambda \delta_{1}, \ldots, \lambda \delta_{n}\right\}$ coincide for some $\lambda>0$. That is, $X$ and $Y$ are quasisymmetrically equivalent if and only if they are conformally equivalent and bi-Lipschitz equivalent if and only if they are isometrically equivalent. This extends work of Rickman (unpublished) and Tukia [67].

\section{BASIC NOTATION AND DEFINITIONS}

We use the Polish notation $|x-y|$ for the distance function in any metric space. For each $n \geq 1$, we write $\mathbb{R}^{n}$ for the Euclidean space of dimension $n$.

For a set $A$ in a metric space $X$, we write $\bar{A}$ for the closure of $A$ and $\partial A$ for the boundary of $A$. We denote the characteristic function of $A$ by $\chi_{A}$. For $A, B \subset X$ we write $\operatorname{diam} A$ for the diameter of $A$ and $\operatorname{dist}(A, B)$ for the distance from $A$ to $B$. For $x \in X$, we abbreviate $\operatorname{dist}(\{x\}, A)=\operatorname{dist}(x, A)$.

For $x \in X$ and $r>0$, we denote by $B(x, r)=\{y \in X:|x-y|<r\}, \bar{B}(x, r)=$ $\{y \in X:|x-y| \leq r\}$, and $S(x, r)=\{y \in X:|x-y|=r\}$ denote the open ball, closed ball and sphere with center $x$ and radius $r$. For any ball $B$ with center $x$ and radius $r$, the notation $C B$ denotes the dilated ball with center $x$ and radius $C r$. In general metric spaces, the center and radius of a given ball need not be uniquely specified. Whenever we write $C B$ for the dilation of a ball $B$ by the factor $C$, it will always be understood that $B$ comes equipped with a particular choice of center and radius.

For $A \subset X$ and $\epsilon>0$ we define the $\epsilon$-neighborhood of $A$ to be

$$
B(A, \epsilon)=\bigcup_{x \in A} B(x, \epsilon)=\{y \in X: \operatorname{dist}(y, A)<\epsilon\} .
$$

Every closed ball $\bar{B}(x, r)$ in a metric space $X$ satisfies $\operatorname{diam} \bar{B}(x, r) \leq 2 r$. No uniform lower bound for the diameter need hold in general as $x$ may be an isolated point of $X$. We say that $X$ is $c_{1}$-uniformly perfect, $0<c_{1} \leq 1$, if

$$
\operatorname{diam} \bar{B}(x, r) \geq 2 c_{1} r
$$

for each closed ball $\bar{B}(x, r) \subsetneq X$. For example, every connected space is $\frac{1}{2}$ uniformly perfect and every space which admits a $Q$-regular measure for some $Q>0$ with regularity constants $c_{0}>0$ and $C_{0}<\infty$ is $c_{1}$-uniformly perfect for all $c_{1}<\left(C_{0} / c_{0}\right)^{-1 / Q}$. Uniformly perfect metric spaces contain no isolated points.

We say that a metric space is locally compact if every point has a precompact neighborhood. If $E$ is a compact subset of a locally compact metric space, then there exists $\delta>0$ so that $B(E, \delta)$ is precompact; this can be proved by a straightforward application of the Lebesgue number lemma [74, Theorem 22.5] on $E$. 
By a continuum we mean a compact, connected set; a continuum is nondegenerate if it contains at least two points. A metric space $X$ is called $C$-linearly locally connected $(C-L L C), C \geq 1$, if every pair of points in a closed ball $\bar{B}(x, r) \subset X$ can be joined by a continuum which lies in $\bar{B}(x, C r)$ and every pair of points in $X \backslash B(x, r)$ can be joined by a continuum which lies in $X \backslash B(x, r / C)$. If these two conditions hold only for $0<r<r_{0}=r_{0}(x)$, we call $X$ locally $C$ - $L L C$.

A homeomorphism $f: X \rightarrow Y$ between metric spaces is $L$-bi-Lipschitz, $L \geq 1$, if

$$
|x-y| / L \leq|f x-f y| \leq L|x-y|
$$

for all $x, y \in X$. Recalling a definition from the introduction, we say that $f$ is $\eta$-quasisymmetric, where $\eta:[0, \infty) \rightarrow[0, \infty)$ is an increasing homeomorphism, if

$$
|f x-f y| \leq \eta(t)|f x-f z|
$$

whenever $x, y, z \in X$ and $t>0$ satisfy $|x-y| \leq t|x-z|$. Also, we say that $f$ is locally $\eta$-quasisymmetric if each point in $X$ has a neighborhood on which $f$ is $\eta$-quasisymmetric.

We denote by $C, c, \ldots$ various unspecified constants whose values may change, even within a given line. For the most part, we observe the following useful convention: we write $C$ when we wish to emphasize that a certain constant is finite and we write $c$ when we wish to emphasize that it is nonzero.

By a curve in a metric space $X$ we mean a continuous map $\gamma$ from an interval $I$ into $X$. To avoid certain technical problems, we will always exclude the case when $\gamma$ is constant. For the basic theory of rectifiable and locally rectifiable curves in Euclidean space or general metric spaces, we refer the reader to [71, Chapter 1] [38. Section 2]. For a subinterval $I_{0} \subset I$, we write $\gamma_{0}=\left.\gamma\right|_{I_{0}}$. We will usually be careful to distinguish between the curve (which is the map $\gamma: I \rightarrow X$ ) and the image set $\gamma(I)$ in $X$, which we will denote by $|\gamma|$. A metric space is called rectifiably connected if every pair of points can be joined by a rectifiable curve.

We say that a measure $\mu$ on a metric space $X$ is Borel if Borel sets are measurable and Borel regular if every measurable set is contained in a Borel set of equal measure. Also, we say that $\mu$ is locally finite if $\mu(K)<\infty$ for each bounded set $K \subset X$. (See [49] Chapter 1] or [26, Chapter 2] for further information regarding measures in general metric spaces.)

For the sake of completeness, we repeat here the following definition which was given in the introduction.

Definition 1.1. Let $Q \geq 0$ and let $\mu$ be a Borel measure on $X$. We say that $\mu$ satisfies upper mass bounds with exponent $Q$ if there exists $C_{0}<\infty$ so that

$$
\mu(\bar{B}(x, r)) \leq C_{0} r^{Q}
$$

for all $x \in X$ and $r>0$. Similarly, we say that $\mu$ satisfies lower mass bounds with exponent $Q$ if there exists $c_{0}>0$ so that

$$
\mu(\bar{B}(x, r)) \geq c_{0} r^{Q}
$$

for all $x \in X$ and $0<r<\operatorname{diam} X$. If the measure $\mu$ satisfies both (1.2) and (1.3) with exponent $Q$, we say that $\mu$ is an (Ahlfors-David) $Q$-regular measure on $X$ and that $X$ is (Ahlfors-David) $Q$-regular. The constants $c_{0}$ and $C_{0}$ are called the regularity data of $X$. 
It is easy to see [61, Lemma C.3] that if a metric space is $Q$-regular for some Borel measure $\mu$, then it has Hausdorff dimension equal to $Q$, in fact, $\mu$ is comparable with the Hausdorff measure $\mathcal{H}_{Q}$ (cf. Proposition 4.10). (For the definition of Hausdorff measures and dimensions, see Section 3]) Thus the choice of which $Q$-regular measure to consider on a given $Q$-regular space is (for our purposes) irrelevant.

Let $X$ be a metric space and let $\mu$ be a locally finite Borel measure on $X$. Let $\Gamma$ be a family of curves in $X$ and let $p \geq 1$. We denote by $\operatorname{Mod}_{p} \Gamma$ the $p$-modulus of $\Gamma$, equal to the infimum of the values $\int_{X} \rho^{p} d \mu$ over all Borel measurable functions $\rho: X \rightarrow[0, \infty]$ for which $\int_{\gamma} \rho d s \geq 1$ for each locally rectifiable curve $\gamma \in \Gamma$. Such a function $\rho$ is called admissible for the curve family $\Gamma$ (see [71. Chapter 1, Section 6] or [38, Section 2.3]). We will occasionally use the term classical modulus to distinguish $\operatorname{Mod}_{p}$ from the generalized modulus defined in section 7.7. In an Ahlfors $Q$-regular space, we also call $\operatorname{Mod}_{Q}$ the conformal modulus. The term arises because the $n$-modulus is a conformal invariant in $\mathbb{R}^{n}$.

We say that a property $\mathcal{P}$ of curves holds for $p$-almost every curve, $p \geq 1$, if the collection $\Gamma$ of curves for which $\mathcal{P}$ fails to hold satisfies $\operatorname{Mod}_{p} \Gamma=0$.

Fundamental properties of the $p$-modulus are collected in the following proposition.

Proposition 1.4. Let $X, \mu$ and $p$ be as above. Then

(i) $\operatorname{Mod}_{p} \emptyset=0$;

(ii) if $\Gamma \subset \Gamma^{\prime}$, then $\operatorname{Mod}_{p} \Gamma \leq \operatorname{Mod}_{p} \Gamma^{\prime}$;

(iii) if $\Gamma=\bigcup_{j} \Gamma_{j}$, then $\operatorname{Mod}_{p} \Gamma \leq \sum_{j=1}^{\infty} \operatorname{Mod}_{p} \Gamma_{j}$;

(iv) if every curve $\gamma \in \Gamma$ contains a subcurve $\gamma^{\prime} \in \Gamma^{\prime}$ (in which case we say that $\Gamma$ is minorized by $\left.\Gamma^{\prime}\right)$, then $\operatorname{Mod}_{p} \Gamma \leq \operatorname{Mod}_{p} \Gamma^{\prime}$;

(v) $\Gamma$ has vanishing $p$-modulus if and only if there exists $\rho \in L^{p}(X, \mu)$ so that $\int_{\gamma} \rho d s=\infty$ for each $\gamma \in \Gamma$

(vi) for any curve family $\Gamma$, the infimum in the definition of $\operatorname{Mod}_{p} \Gamma$ is unchanged if we restrict to the family of lower semicontinuous admissible metrics $\rho$;

(vii) if $\rho_{1}, \rho_{2}, \ldots$ is a sequence of Borel functions converging to $\rho$ in $L^{p}(X, \mu)$, then there exists a subsequence $\rho_{j_{1}}, \rho_{j_{2}}, \ldots$ so that $\int_{\gamma}\left|\rho_{j_{l}}-\rho\right| d s \rightarrow 0$ for p-almost every curve $\gamma$;

(viii) when $p>1$, extremal admissible metrics exist $p$-a.e., i.e., for each curve family $\Gamma$ there is a subfamily $\Gamma^{\prime} \subset \Gamma$ with $\operatorname{Mod}_{p}\left(\Gamma \backslash \Gamma^{\prime}\right)=0$ and a Borel function $\rho^{\prime} \in L^{p}(X, \mu)$ admissible for $\Gamma^{\prime}$ which satisfies $\int_{X}\left(\rho^{\prime}\right)^{p} d \mu=\operatorname{Mod}_{p} \Gamma^{\prime}$;

(ix) if $1<p<\infty$ and $\Gamma_{1} \subset \Gamma_{2} \subset \cdots$ is an increasing sequence of curve families with $\Gamma=\bigcup_{j=1}^{\infty} \Gamma_{j}$, then $\operatorname{Mod}_{p} \Gamma=\lim _{j \rightarrow \infty} \operatorname{Mod}_{p} \Gamma_{j}$.

For (i)-(iv), see e.g. [71, Theorems 6.2 and 6.4]; for (v) see [27, Theorem 2]. (vi) follows from the Vitali-Carathéodory theorem (every function $f$ in $L^{p}(X, \mu)$ can be approximated from above in $L^{p}$ by a sequence of lower semicontinuous functions, see [59, Theorem 2.25]) which in turn makes use of the outer regularity of $\mu$ (every Borel set can be approximated from outside in measure by a sequence of open sets, see [26, 2.2.2])4 For (vii), see [27, Theorem 3(f)]. (viii) follows from the uniform

\footnotetext{
${ }^{4}$ We note in passing that the Vitali-Carathéodory theorem stated above is usually formulated only in the setting of locally compact metric spaces, however, for nonnegative functions $f$ it holds without the assumption of local compactness since the outer regularity of locally finite Borel measures holds in general metric spaces by [26, 2.2.2].
} 
convexity of the Banach space $L^{p}(X, \mu), 1<p<\infty$, see e.g. [27, p. 182]. Finally, (ix) is established (for $X=\mathbb{R}^{n}$ ) in [75] or [31, Section 1, Theorem 7]; the general case is no more difficult.

\section{PART 1. HaUsdorfF AND PACKING MEASURES in GENERAL METRIC SPACES}

In the following four sections we describe in detail several methods for constructing Borel regular measures in arbitrary metric spaces: Carathéodory's construction (Methods I and II) which leads to Hausdorff-type measures and Thomson's construction (Method III) which leads to packing-type measures. We also describe a variation of the notion of the line integral along a (possibly nonrectifiable) curve. The basic idea in all of these constructions is well-known: starting from an arbitrary function $\psi$ defined on a class $S$ of subsets of a metric space $X$, one constructs outer measures on $X$ by optimizing certain sums of values of $\psi$ over subcollections of $S$.

These constructions are most commonly used in the case where $S$ is the family of metric balls in $X$ and $\psi$ is a gauge function, i.e., $\psi(B)$ depends only on the radius of $B$ (see, e.g., [58, Chapter 2] or [49, Chapters 4 and 5]). In contrast, we work throughout with general set functions rather than just gauge functions. This choice is made necessary in view of our application of these measures in the next section. More precisely, we will define a generalized notion of the $p$-modulus of a curve family in terms of measures of this type. The use of general (nongauge) set functions will be an essential ingredient in this definition (see (8.11)).

\section{QuASIROUND SETS}

Throughout this section, $X$ denotes an arbitrary metric space containing at least two points and $k \geq 1$ denotes a fixed real number.

Definition 2.1. Let $A \subset X$ be a closed set. When $k>1$, we call $A$ a $k$-quasiround set if there exist $x \in X$ and $0<r_{1} \leq r_{2}<k r_{1}<\infty$ so that

$$
\bar{B}\left(x, r_{1}\right) \subset A \subset \bar{B}\left(x, r_{2}\right) \text {. }
$$

By decreasing $r_{1}$ if necessary (but keeping $r_{2}<k r_{1}$ ), we may always assume that $r_{2}-r_{1} \geq c r_{1}$ for some $c=c(k)>0$ (say $c=(k-1) / 2$ ). We will make occasional use of this fact (Lemma 5.5 and Proposition 8.2).

We call $x$ a center of $A$ and we call $r_{2}=r_{2}(A)$, resp. $r_{1}=r_{1}(A)$, an outer, resp. inner radius of $A$.

The above definition is vacuous in the case $k=1$. We call a set $A$ a 1-quasiround (or round) set if it is a closed ball $\bar{B}(x, r)$ for some $x$ and $r$.

For $k \geq 1$, we denote by $\mathcal{B}_{k}(X)$ the collection of all $k$-quasiround sets in $X$ and we denote by $\mathcal{B}(X)=\bigcup_{k \geq 1} \mathcal{B}_{k}(X)$ the collection of all quasiround sets.

Remarks 2.3. (1) A closed set $A$ is an element of $\mathcal{B}(X)$ if and only if it is bounded and has nonempty interior.

(2) The center and outer and inner radii of a quasiround set will in general not be uniquely defined (consider the case $X=\mathbb{R}^{n}$ ). (See Lemma 2.4.) Moreover, the collections of centers and radii of a given quasiround set will in general depend on the choice of $k$. That is, a $k$-quasiround set $A$ is an $l$-quasiround set for each $l>k$, but the collections of centers and radii of $A$ when viewed as an $l$-quasiround set will in general be larger than the corresponding collections when $A$ is viewed as a $k$-quasiround set. 
The following lemma will be used in section 3 to show Borel regularity of packingtype measures:

Lemma 2.4. Fix $k>1$ and $A \in \mathcal{B}_{k}(X)$. The set $Z(A)$ of centers of $A$ is an open subset of $X$.

Proof. Let $x \in Z(A)$ and choose $0<r_{1} \leq r_{2}<k r_{1}<\infty$ so that (2.2) holds. Set

$$
\delta=\frac{k r_{1}-r_{2}}{2(k+1)}>0 \text {. }
$$

For $x^{\prime} \in B(x, \delta)$, set $r_{1}^{\prime}=r_{1}-\delta$ and $r_{2}^{\prime}=r_{2}+\delta$. One easily verifies that (2.2) holds with $x, r_{1}, r_{2}$ replaced by $x^{\prime}, r_{1}^{\prime}, r_{2}^{\prime}$. Thus $x^{\prime}$ is also a center of $A$. Furthermore, $r_{1}^{\prime} \leq r_{2}^{\prime}<k r_{1}^{\prime}$ by the choice of $\delta$.

The classes $\mathcal{B}_{k}(X)$ interact naturally with mappings satisfying conditions of "bounded distortion" such as quasisymmetry and (metric) quasiconformality. This connection will be explored in greater detail in section 9, for now we content ourselves with a simple lemma which illustrates the idea. (See also [69, Lemma 3.1].)

Lemma 2.5. Let $f: X \rightarrow Y$ be an $\eta$-quasisymmetric homeomorphism between metric spaces. If $A \in \mathcal{B}_{k}(X), k \geq 1$, then $f(A) \in \mathcal{B}_{k^{\prime}}(Y)$ for each $k^{\prime}>\eta(k)$.

Note that $\eta(k) \geq 1$ since $X$ contains at least two points.

Proof. Let $A \in \mathcal{B}_{k}(X)$ and choose $x \in X$ and $0<r_{1} \leq r_{2}<k r_{1}<\infty$ so that $\bar{B}\left(x, r_{1}\right) \subset A \subset \bar{B}\left(x, r_{2}\right)$. If $\bar{B}\left(x, r_{1}\right)=X$, then $X$ is bounded and hence $Y$ is also bounded, in which case $Y$ is equal to a metric ball and hence is in $\mathcal{B}_{1}(Y) \subset \mathcal{B}_{k^{\prime}}(Y)$. Similarly, if $A=\{x\}$, then $x$ is isolated in $X$ and hence $f(x)$ is isolated in $Y$, in which case $f(A)=\{f(x)\}=\bar{B}\left(f(x), r^{\prime}\right) \in \mathcal{B}_{1}(Y) \subset \mathcal{B}_{k^{\prime}}(Y)$ for some small $r^{\prime}>0$.

Thus we may assume that $\bar{B}\left(x, r_{1}\right)$ is a proper subset of $X$ and that $A \neq\{x\}$. Next, by replacing $r_{1}$ and $r_{2}$ with $\lambda r_{1}$ and $\lambda r_{2}$ if necessary, we may assume that there exists a point $y \in A \cap S\left(x, r_{2}\right)$ (recall that we assume that $A$ is closed). Define

$$
r_{2}^{\prime}:=\sup \left\{|f(y)-f(x)|: y \in \bar{B}\left(x, r_{2}\right)\right\}
$$

and

$$
r_{1}^{\prime}:=(1-\epsilon) \inf \left\{|f(z)-f(x)|: z \notin \bar{B}\left(x, r_{1}\right)\right\},
$$

where $\epsilon>0$ is chosen so small that $\eta(k)<(1-\epsilon) k^{\prime}$. The existence of the point $y$ mentioned above implies that $r_{1}^{\prime} \leq r_{2}^{\prime}$ and the quasisymmetry of $f$ implies that $r_{2}^{\prime}<k^{\prime} r_{1}^{\prime}$. Finally, it is easy to check that $\bar{B}\left(f(x), r_{1}^{\prime}\right) \subset f(A) \subset \bar{B}\left(f(x), r_{2}^{\prime}\right)$.

Definition 2.6. Let $E \subset X$, let $\mathcal{V}$ be a collection of quasiround subsets of $X$, and let $\mathcal{C} \subset \mathcal{V}$.

We say that $\mathcal{V}$ is a fine (or Vitali) cover of $E$ if for each $x \in E$ and $\epsilon>0$, there is an element $A \in \mathcal{V}$ which is centered at $x$ and has an outer radius $r_{2}(A)<\epsilon$.

We say that $\mathcal{C}$ is a $\mathcal{V}$-full cover of $E$ if there exists a function $\Delta: E \rightarrow(0, \infty)$ so that every element $A \in \mathcal{V}$ which is centered at $x$ and has an outer radius $r_{2}(A) \leq \Delta(x)$ is an element of $\mathcal{C}$.

We abbreviate the phrase "fine cover of $X$ " to "fine collection".

Informally, $\mathcal{V}$ is a fine cover of $E$ if it contains arbitrarily small sets centered at each point of $E$, while $\mathcal{C} \subset \mathcal{V}$ is a $\mathcal{V}$-full cover of $E$ if all sufficiently small elements of $\mathcal{V}$ centered at points of $E$ are elements of $\mathcal{C}$. 
Example 2.7. Let $\mathcal{V}$ be a fine collection of quasiround sets in $X$ and let $E \subset X$. For $\delta>0$, let $\mathcal{V}^{\delta}(E)$ denote the collection of sets $A \in \mathcal{V}$ which are centered at some point of $E$ and have an outer radius $r_{2}(A) \leq \delta$. Then $\mathcal{V}^{\delta}(E)$ is a $\mathcal{V}$-full cover of $E$. The full cover function $\Delta: E \rightarrow(0, \infty)$ can be taken to be the constant function $\Delta(x)=\delta$.

Note that we do not assume continuity (or even semicontinuity) for the full cover function $\Delta$ in Definition 2.6. This will be crucial in our proof that the packing measure is an outer measure (see part (iii) of the following proposition).

Proposition 2.8. Let $\mathcal{V} \subset \mathcal{B}(X)$ be a fine collection and let $E, E_{1}, E_{2}, \ldots \subset X$. Then

(i) every $\mathcal{V}$-full cover of $E$ is also a fine cover of $E$;

(ii) the intersection of two $\mathcal{V}$-full covers of $E$ is a $\mathcal{V}$-full cover of $E$ and the intersection of a $\mathcal{V}$-full cover of $E$ with a fine cover of $E$ which consists of elements of $\mathcal{V}$ is a fine cover of $E$; 5

(iii) if $\mathcal{C}_{n}$ is a $\mathcal{V}$-full, resp. fine cover of $E_{n}$ for each $n \in \mathbb{N}$, then $\bigcup_{n=1}^{\infty} \mathcal{C}_{n}$ is a $\mathcal{V}$-full, resp. fine, cover of $\bigcup_{n=1}^{\infty} E_{n}$

(iv) if $\mathcal{C}$ is a $\mathcal{V}$-full, resp. fine cover of $E$ and $G \supset E$ is an open set, then

$$
\mathcal{C}(G):=\{A \in \mathcal{C}: A \subset G\}
$$

is again a $\mathcal{V}$-full, resp. fine cover of $E$.

These properties are given as exercises in section 3.9 of [11] we leave the proofs to the reader. As an example, we give the proof for full covers in case (iv). For $x \in E$, choose $r=r(x)>0$ so that $\bar{B}(x, r) \subset G$. If $\Delta: E \rightarrow(0, \infty)$ is the full cover function for $\mathcal{C}$, then we may take $\Delta^{\prime}(x)=\min \{\Delta(x), r(x)\}$ to be the full cover function for the collection $\mathcal{C}(G)$.

\section{Constructions of measures}

We describe several methods for constructing measures on a metric space $X$. In each of these methods, we start with an arbitrary function $\psi$ defined on a fine collection $\mathcal{V} \subset \mathcal{B}_{k}(X), k \geq 1$, and make suitable refinements to arrive at an outer measure on $X$. We emphasize throughout the dependence of the resulting measure on the choice of the parameter $k$ and the collection $\mathcal{V}$. Since we will make use of various choices of $k$ and $\mathcal{V}$, our notation will always make this dependence clear.

For the purposes of this section, assume that we have fixed a real number $k \geq 1$ and a fine collection $\mathcal{V} \subset \mathcal{B}_{k}(X)$.

Definition 3.1. Let $\psi: \mathcal{V} \rightarrow[0, \infty]$ be a set function. For $E \subset X$ and $0<\delta \leq \infty$, set

$$
\Psi_{k, \mathcal{V}}^{\delta}(E)=\inf \sum_{i} \psi\left(A_{i}\right)
$$

the infimum being taken over all finite or countable collections $\left\{A_{1}, A_{2}, \ldots\right\} \subset \mathcal{V}$ which cover $E$ and have outer radii $r_{2}\left(A_{i}\right) \leq \delta$ for all $i$. Then, set

$$
\Psi_{k, \mathcal{V}}(E)=\lim _{\delta \rightarrow 0} \Psi_{k, \mathcal{V}}^{\delta}(E)
$$

\footnotetext{
${ }^{5}$ The intersection of two fine covers of $E$, of course, need not be a fine cover of $E$; it may be the empty collection.
} 
The function $\Psi_{k, \mathcal{V}}^{\infty}$ is called the Method I outer measure and $\Psi_{k, \mathcal{V}}$ is called the Method II outer measure constructed from the data $(k, \mathcal{V}, \psi)$. We also call $\Psi_{k, \mathcal{V}}$ the Carathéodory $\psi$-measure.

When $\mathcal{V}=\mathcal{B}_{k}(X)$ we abbreviate $\Psi_{k, \mathcal{V}}=\Psi_{k}$

It is elementary to verify that both $\Psi_{k, \mathcal{V}}^{\infty}$ and $\Psi_{k, \mathcal{V}}$ are outer measures on $X$. The reason for introducing Method II is that the outer measure $\Psi_{k, \mathcal{V}}^{\infty}$ need not be a Borel measure, while $\Psi_{k, \mathcal{V}}$ is always Borel regular. For proofs of these facts, see [49, Theorem 4.2] or [26, 2.10.1].

Example 3.3. For $0 \leq \alpha<\infty$, define $\psi: \mathcal{B}_{1}(X) \rightarrow[0, \infty]$ on the closed balls in $X$ by $\psi(\bar{B}(x, r))=r^{\alpha}$. We call the measure $\Psi_{1}$ constructed by Method II the Hausdorff $\alpha$-measure on $X$ and denote it by $\mathcal{H}_{\alpha} 6$ The outer measure $\Psi_{1}^{\infty}$ constructed by Method I is called the Hausdorff $\alpha$-content and denoted $\mathcal{H}_{\alpha}^{\infty}$.

Recall that the Hausdorff dimension of a set $E \subset X$ is the value

$$
\operatorname{dim}_{H} E=\inf \left\{\alpha \mid \mathcal{H}_{\alpha}(E)=0\right\}=\sup \left\{\alpha \mid \mathcal{H}_{\alpha}(E)=\infty\right\},
$$

where we interpret $\sup \emptyset=0$. The Hausdorff dimension is monotonic $(E \subset F$ implies $\left.\operatorname{dim}_{H} E \leq \operatorname{dim}_{H} F\right)$ and countably stable $\left(E=\bigcup_{i=1}^{\infty} E_{i} \operatorname{implies}_{\operatorname{dim}_{H}} E=\right.$ $\left.\sup _{i} \operatorname{dim}_{H} E_{i}\right)$. For each Borel set $E$ in $X, \operatorname{dim}_{H} E \geq \operatorname{dim}_{T} E$, where $\operatorname{dim}_{T} E$ denotes the topological dimension of $E$ (see e.g. 41]).

Remark 3.4. The Carathéodory construction respects multiplication by scalars (the measure constructed from $a \cdot \psi, a \geq 0$, is $\left.a \cdot \Psi_{k, \mathcal{V}}\right)$ as well as the standard partial ordering on set functions defined on the class $\mathcal{V}\left(\psi(A) \leq \psi^{\prime}(A)\right.$ for all sufficiently small sets $A \in \mathcal{V}$ implies $\left.\Psi_{k, \mathcal{V}} \leq \Psi_{k, \mathcal{V}}^{\prime}\right)$. However, in general this construction need not respect addition, i.e., the measure $\Xi_{k, \mathcal{V}}$ constructed from $\xi=\psi+\varphi$ need not be related to $\Psi_{k, \mathcal{V}}$ and $\Phi_{k, \mathcal{V}}$. The packing measure, which we define next, will be better behaved in this regard.

Definition 3.5. Let $k, \mathcal{V}$ and $\psi$ be as before. We define the variation of $\psi$ over a collection $\mathcal{C} \subset \mathcal{V}$ to be

$$
V(\psi, \mathcal{C})=\sup \sum_{i} \psi\left(A_{i}\right)
$$

where the supremum is taken over all finite or countable collections $A_{1}, A_{2}, \ldots$ consisting of pairwise disjoint elements of $\mathcal{C}$. If no such packings exist (which only occurs if $\mathcal{C}$ is the empty collection), we write $V(\psi, \mathcal{C})=0$.

It is easy to verify that the variation is monotonic:

$$
\mathcal{C} \subset \mathcal{C}^{\prime} \Rightarrow V(\psi, \mathcal{C}) \leq V\left(\psi, \mathcal{C}^{\prime}\right)
$$

and countably subadditive:

$$
V\left(\psi, \bigcup_{k=1}^{\infty} \mathcal{C}_{k}\right) \leq \sum_{k=1}^{\infty} V\left(\psi, \mathcal{C}_{k}\right)
$$

We use this notion to define packing measures in general metric spaces. Method III was introduced by Thomson [65], following earlier ideas of Henstock. For further information, see [24].

\footnotetext{
${ }^{6}$ More precisely, $\mathcal{H}_{\alpha}$ is what is usually called the spherical Hausdorff $\alpha$-measure since we have defined it using balls $\bar{B}(x, r)$ rather than general sets.
} 
Definition 3.6. For $E \subset X$, set

$$
\Psi_{k, \mathcal{V}}^{\bullet}(E)=\inf _{\mathcal{C}} V(\psi, \mathcal{C})
$$

where the infimum is taken over all $\mathcal{V}$-full covers $\mathcal{C}$ of $E \nabla$ Note that such covers always exist by Example 2.7. We call $\Psi_{k, \mathcal{V}}^{\bullet}$ the Method III outer measure constructed from the data $(k, \mathcal{V}, \psi)$ or the packing $\psi$-measure.

As before, we abbreviate $\Psi_{k, \mathcal{B}_{k}(X)}^{\bullet}=\Psi_{k}^{\bullet}$.

Proposition 3.8. If $k>1$, then $\Psi_{k, \mathcal{V}}^{\bullet}$ is a Borel regular measure on $X$.

Compare [11, Theorems 3.29 and 3.30].

Note here the restriction $k>1$. When $k=1$ we can only show in general that the packing measure is a Borel measure (see, however, Example 3.11).

Proof. We begin by verifying that $\Psi_{k, \mathcal{V}}^{\bullet}$ is an outer measure. The empty collection $\mathcal{N}$ is a $\mathcal{V}$-full cover of the empty set with $V(\psi, \mathcal{N})=0$, whence $\Psi_{k, \mathcal{V}}^{\bullet}(\emptyset)=0$. Monotonicity of $\Psi_{k, \mathcal{V}}^{\bullet}$ follows from monotonicity of the variation, since every $\mathcal{V}$ full cover of a set $E$ is also a $\mathcal{V}$-full cover of each subset of $E$. Finally, countable subadditivity for $\Psi_{k, \mathcal{V}}^{\bullet}$ follows from the corresponding result for the variation and Proposition 2.8(iii).

Next, we verify that $\Psi_{k, \mathcal{V}}^{\bullet}$ is a Borel measure. Recall [26, 2.3.2(9)] that this holds if and only if the Carathéodory condition is satisfied:

$$
\Psi_{k, \mathcal{V}}^{\bullet}(E \cup F)=\Psi_{k, \mathcal{V}}^{\bullet}(E)+\Psi_{k, \mathcal{V}}^{\bullet}(F)
$$

whenever $\operatorname{dist}(E, F)>0$. Suppose then that $E, F \subset X$ satisfy $\operatorname{dist}(E, F)>0$. Let $\mathcal{C}$ be an arbitrary $\mathcal{V}$-full cover of $E \cup F$. Choose a pair of disjoint open sets $G_{1} \supset E$ and $G_{2} \supset F$. By Proposition 2.8(iv), the families $\mathcal{C}_{1}=\left\{A \in \mathcal{C} \mid A \subset G_{1}\right\}$ and $\mathcal{C}_{2}=\left\{A \in \mathcal{C} \mid A \subset G_{2}\right\}$ are full covers of $E$ and $F$, respectively. Since no set in $\mathcal{C}_{1}$ meets any set in $\mathcal{C}_{2}$,

$$
\Psi_{k, \mathcal{V}}^{\bullet}(E)+\Psi_{k, \mathcal{V}}^{\bullet}(F) \leq V\left(\psi, \mathcal{C}_{1}\right)+V\left(\psi, \mathcal{C}_{2}\right) \leq V(\psi, \mathcal{C})
$$

Now (3.9) follows by taking the infimum over all such covers $\mathcal{C}$. (This part of the proof is valid for any $k \geq 1$.)

Assume now that $k>1$; we show that $\Psi_{k, \mathcal{V}}^{\bullet}$ is Borel regular. Let $E \subset X$; without loss of generality we may assume that $\Psi_{k, \mathcal{V}}^{\bullet}(E)$ is finite. Let $m \in \mathbb{N}$ and let $\mathcal{C}^{m}$ be a $\mathcal{V}$-full cover of $E$ with $V\left(\psi, \mathcal{C}^{m}\right)<\Psi_{k, \mathcal{V}}^{\bullet}(E)+1 / m$. For $n \in \mathbb{N}$, set

$$
E_{n}^{m}=\left\{x \in E: \Delta^{m}(x)>1 / n\right\}
$$

where $\Delta^{m}: E \rightarrow(0, \infty)$ is the full cover function for $\mathcal{C}^{m}$.

For fixed $m$, consider the increasing sequence of closed sets $\overline{E_{n}^{m}}$. Let $x \in \overline{E_{n}^{m}}$ and let $A \in \mathcal{V}$ be a quasiround set centered at $x$ with an outer radius $r_{2}(A) \leq 1 / n$. Since the set $Z(A)$ of centers of $A$ is open (Lemma 2.4), there exists a center $y$ for $A$ for which $y \in E_{n}^{m}$. Thus $A \in \mathcal{C}^{m}$ and so $\mathcal{C}^{m}$ is a $\mathcal{V}$-full cover of $\overline{E_{n}^{m}}$ (the constant function $1 / n$ is a full cover function for $\mathcal{C}^{m}$ on the set $\overline{E_{n}^{m}}$ ). We conclude that

$$
\Psi_{k, \mathcal{V}}^{\bullet}\left(\overline{E_{n}^{m}}\right) \leq V\left(\psi, \mathcal{C}^{m}\right)<\Psi_{k, \mathcal{V}}^{\bullet}(E)+1 / m
$$

for each $m$ and $n$. Then $F=\bigcap_{m=1}^{\infty} \bigcup_{n=1}^{\infty} \overline{E_{n}^{m}}$ is a Borel set containing $E$ with $\Psi_{k, \mathcal{V}}^{\bullet}(F)=\Psi_{k, \mathcal{V}}^{\bullet}(E)$.

\footnotetext{
${ }^{7}$ By Proposition 2.8(ii), the infimum in (3.7) can also be viewed as a Moore-Smith limit along the net of full covers partially ordered by reverse inclusion.
} 
Example 3.11. Let $\mathcal{V}=\mathcal{B}_{1}(X), \alpha$ and $\psi$ be as in Example 3.3. We call the measure $\Psi_{1, \mathcal{V}}^{\bullet}$ constructed by Method III the packing $\alpha$-measure on $X$ and denote it by $\mathcal{P}_{\alpha}$.

In this situation, the particularly nice form of the set function $\psi$ compensates for the loss of flexibility inherent in the choice $k=1$ and we are again able to show that $\mathcal{P}_{\alpha}$ is Borel regular (see Remark 3.13).

As was the case for the Hausdorff measures, $\mathcal{P}_{\alpha}(E)<\infty \Rightarrow \mathcal{P}_{\beta}(E)=0$ whenever $\alpha<\beta$. We define the packing dimension of a set $E \subset X$ to be

$$
\operatorname{dim}_{P} E=\inf \left\{\alpha \mid \mathcal{P}_{\alpha}(E)=0\right\}=\sup \left\{\alpha \mid \mathcal{P}_{\alpha}(E)=\infty\right\}
$$

where again we interpret $\sup \emptyset=0$. As before, the packing dimension is monotonic and countably stable.

Remark 3.12. Method III also respects scalar multiplication and the standard partial ordering on functions $\psi$. Furthermore, the assignment $\psi \mapsto \Psi_{k, \mathcal{V}}^{\bullet}$ is finitely subadditive: if $\varphi, \psi: \mathcal{V} \rightarrow[0, \infty]$ and $\xi=\varphi+\psi$, then

$$
\Xi_{k, \mathcal{V}}^{\bullet}(E) \leq \Phi_{k, \mathcal{V}}^{\bullet}(E)+\Psi_{k, \mathcal{V}}^{\bullet}(E)
$$

for $E \subset X$. This follows from Proposition 2.8(ii).

Remark 3.13. Definition 3.6 is not the standard definition of packing measures as considered by Tricot, Taylor, St. Raymond, and others [66], 64], 60] but it leads to the same measure in the case when $\mathcal{V}=\mathcal{B}_{1}(X)$ consists of the closed balls and $\psi(\bar{B}(x, r))=g(r)$ for some gauge function $g$. This was shown by Edgar in [25] and 23]. For the sake of completeness, we give a brief recapitulation of his results here.

For our purposes, a gauge function is defined to be an increasing, continuous function $g$ from the interval $[0, \infty)$ to itself which satisfies $g(0)=0$.

For $E \subset X$, let

$$
\widehat{\mathcal{P}}_{g}^{\bullet}(E)=\lim _{\delta \rightarrow 0} V\left(g, \mathcal{B}_{1}^{\delta}(E)\right)=\inf _{\delta>0} V\left(g, \mathcal{B}_{1}^{\delta}(E)\right) .
$$

(Recall that $\mathcal{B}_{1}^{\delta}(E)$ denotes the collection of closed balls with radius at most $\delta$ which are centered at a point of $E$.) The set function $\widehat{\mathcal{P}}_{g}^{\bullet}$ is monotonic and assigns the value zero to the empty set, but need not be countably subadditive. We introduce this additional property by applying Method I, that is, we define

$$
\widetilde{\mathcal{P}}_{g}^{\bullet}(E)=\inf \sum_{m} \widehat{\mathcal{P}}_{g}^{\bullet}\left(F_{m}\right)
$$

where the infimum is taken over all collections $\left\{F_{1}, F_{2}, \ldots\right\}$ covering $E$. Then $\widetilde{\mathcal{P}}_{g}^{\bullet}$ is a Borel measure on $X$ [23, p. 168].

For any gauge function $g$ and any set $E$ in $X, \widehat{\mathcal{P}}_{g}^{\bullet}(E)=\widehat{\mathcal{P}}_{g}^{\bullet}(\bar{E})$ (cf. 25, Proposition 1.10]). Indeed, if $\bar{B}(x, r)$ is a ball with $x \in \bar{E}$, we may choose $x^{\prime} \in E$ as close as we please to $x$ and set $r^{\prime}=r-\left|x-x^{\prime}\right|$. Then $\bar{B}\left(x^{\prime}, r^{\prime}\right) \subset \bar{B}(x, r)$. Since $g$ is assumed to be continuous, for any $\epsilon>0$ and any packing $\left\{\bar{B}\left(x_{i}, r_{i}\right)\right\}_{i=1}^{\infty} \subset \mathcal{B}_{1}^{\delta}(\bar{E})$, we may find a packing $\left\{\bar{B}\left(x_{i}^{\prime}, r_{i}^{\prime}\right)\right\}_{i=1}^{\infty} \subset \mathcal{B}_{1}^{\delta}(E)$ for which

$$
\sum_{i} g\left(r_{i}\right)<\sum_{i} g\left(r_{i}^{\prime}\right)+\epsilon
$$

It follows that $V\left(g, \mathcal{B}_{1}^{\delta}(\bar{E})\right)=V\left(g, \mathcal{B}_{1}^{\delta}(E)\right)$ and so $\widehat{\mathcal{P}}_{g}^{\bullet}(E)=\widehat{\mathcal{P}}_{g}^{\bullet}(\bar{E})$. Thus, in the definition of $\widetilde{\mathcal{P}}_{g}^{\bullet}$, it suffices to take the infimum over collections of closed sets. We conclude that $\widetilde{\mathcal{P}}_{g}^{\bullet}$ is Borel regular. 
For any gauge function $g$ and any Borel set $E$ in $X, \widetilde{\mathcal{P}}_{g}^{\bullet}(E)=\mathcal{P}_{g}^{\bullet}(E)$ (cf. [25] Proposition 1.11]). Indeed, since $\mathcal{B}_{1}^{\delta}(E)$ is a $\mathcal{B}_{1}(X)$-full cover of $E$ for each $\delta>0$, it is clear that $\mathcal{P}_{g}^{\bullet}(E) \leq \widehat{\mathcal{P}}_{g}^{\bullet}(E)$ and so $\mathcal{P}_{g}^{\bullet}(E) \leq \widetilde{\mathcal{P}}_{g}^{\bullet}(E)$. Conversely, if $\mathcal{C}$ is an arbitrary $\mathcal{B}_{1}(X)$-full cover of $E$, define $E_{n}$ as in (3.10) for each $n \in \mathbb{N}$. The sets $E_{1}, E_{2}, \ldots$ increase to $E$ and $\mathcal{B}_{1}^{1 / n}\left(E_{n}\right) \subset \mathcal{C}$ for each $n$. Thus

$$
\widetilde{\mathcal{P}}_{g}^{\bullet}\left(E_{n}\right) \leq \widehat{\mathcal{P}}_{g}^{\bullet}\left(E_{n}\right) \leq V\left(g, \mathcal{B}_{1}^{1 / n}\left(E_{n}\right)\right) \leq V(g, \mathcal{C}) .
$$

By the Borel regularity of the measure $\widetilde{\mathcal{P}}_{g}^{\bullet}$, we may pass to the limit as $n \rightarrow \infty$ (regardless of the measurability of $E_{n}$ ). Taking the infimum over all covers $\mathcal{C}$ completes the proof.

\section{Relations between Hausdorff- and PaCking-type measures}

For completely arbitrary choices of $\mathcal{V}$ and $\psi: \mathcal{V} \rightarrow[0, \infty]$, the measures $\Psi_{k, \mathcal{V}}$ and $\Psi_{k, \mathcal{V}}^{\bullet}$ need not satisfy any relation. Under an additional assumption on the class $\mathcal{V}$ and the set function $\psi$, however, we will show that the Hausdorff-type measure $\Psi_{1, \mathcal{V}}$ is always bounded from above by the packing-type measure $\Psi_{1, \mathcal{V}}^{\bullet}$. Compare [49, Theorem 5.12] and [24, Corollary 1.3.5].

Recall that for any ball $B=\bar{B}(x, r)$ in $X$ and any $t>1$, the notation $t B$ denotes the dilated ball $\bar{B}(x, t r)$. This set is well-defined only if we fix a particular choice of center and radius for the ball $B$. We will always assume that this is the case.

We require the following basic covering lemma [26, 2.8.4-5].

Lemma 4.1. Let $\mathcal{C}$ be a bounded collection of closed balls (i.e., assume that the radii of the balls in $\mathcal{C}$ are uniformly bounded). Then there exists a disjointed subcollection $\mathcal{G}$ of $\mathcal{C}$ so that $\bigcup \mathcal{C} \subset \bigcup 5 \mathcal{G}$.

Here, for a collection $\mathcal{A}$ of subsets of $X$, we write $\bigcup \mathcal{A}$ to denote the union of the sets $A \in \mathcal{A}$ and, for a collection $\mathcal{C}$ of balls in $X$ and a real number $t>1$, we set $t \mathcal{C}=\{t B: B \in \mathcal{C}\}$. By a disjointed collection we mean a collection whose elements are pairwise disjoint.

Proposition 4.2. Let $X$ be a metric space and let $\mathcal{V}$ be a fine collection of closed balls. Let $\psi: \mathcal{V} \cup 5 \mathcal{V} \rightarrow[0, \infty]$. If there exists a constant $M$ so that the collection $\mathcal{V}_{0}$ of balls $B$ in $\mathcal{V}$ satisfying the condition

$$
\psi(5 B) \leq M \psi(B)
$$

is a $\mathcal{V}$-full cover of $X$, then $\Psi_{1, \mathcal{V}}(E) \leq \Psi_{1, \mathcal{V}}^{\bullet}(E)$ for each Borel set $E \subset X$.

Proof. Let $E \subset X$. Without loss of generality, assume that $\Psi_{1, \mathcal{V}}^{\bullet}(E)$ is finite. Let $\delta>0$ and $\epsilon>0$ and let $\mathcal{C}$ be a $\mathcal{V}$-full cover of $E$ for which

$$
V(\psi, \mathcal{C})<\Psi_{1, \mathcal{V}}^{\bullet}(E)+\epsilon .
$$

We may assume without loss of generality that each element of $\mathcal{C}$ has radius at most $\delta$. Let $\left\{B_{1}, B_{2}, \ldots\right\}$ be a disjointed collection in $\mathcal{C}$ with

$$
\sum_{i} \psi\left(B_{i}\right) \leq V(\psi, \mathcal{C})<\sum_{i} \psi\left(B_{i}\right)+\epsilon
$$

Since $V(\psi, \mathcal{C})$ is finite, there exists $k=k(\epsilon)$ so that $\sum_{i=k+1}^{\infty} \psi\left(B_{i}\right)<\epsilon$. Set $G=$ $X \backslash \bigcup_{i=1}^{k} B_{i}$. Since $G$ is an open set in $X$, the collection $\mathcal{C}_{2}=\mathcal{V}_{0} \cap \mathcal{C}(G)$ is a $\mathcal{V}$-full cover of $E \cap G$, recall Proposition [2.8(iv). Using Lemma 4.1, choose a disjointed 
subcollection $\mathcal{G}$ in $\mathcal{C}_{2}$ for which $\bigcup \mathcal{C}_{2} \subset \bigcup 5 \mathcal{G}$. Then $E \backslash \bigcup_{i=1}^{k} B_{i} \subset \bigcup 5 \mathcal{G}$; moreover, the collection $\left\{B_{1}, B_{2}, \ldots, B_{k}\right\} \cup \mathcal{G} \subset \mathcal{C}$ is disjointed. Hence

$$
\sum_{i=1}^{k} \psi\left(B_{i}\right)+\sum_{B \in \mathcal{G}} \psi(B) \leq V(\psi, \mathcal{C})<\sum_{i=1}^{\infty} \psi\left(B_{i}\right)+\epsilon<\sum_{i=1}^{k} \psi\left(B_{i}\right)+2 \epsilon
$$

and so $\sum_{B \in \mathcal{G}} \psi(B)<2 \epsilon$. Since $E \subset B_{1} \cup B_{2} \cup \cdots \cup B_{k} \cup \bigcup 5 \mathcal{G}$, we have

$$
\Psi_{1, \mathcal{V}}^{5 \delta}(E) \leq \sum_{i=1}^{k} \psi\left(B_{i}\right)+\sum_{B \in \mathcal{G}} \psi(5 B) \leq V(\psi, \mathcal{C})+2 M \epsilon \leq \Psi_{1, \mathcal{V}}^{\bullet}(E)+(2 M+1) \epsilon ;
$$

here we have also used the fact that $\mathcal{G} \subset \mathcal{V}_{0}$. Letting $\epsilon$ and $\delta$ tend to zero completes the proof.

Definition 4.4. Let $M>0$. We call the function $\psi$ locally $M$-blanketed if there exists $t>1$ so that the collection $\mathcal{V}_{0}$ of balls in $\mathcal{V}$ which satisfy

$$
\psi(t B) \leq M \psi(B)
$$

is a $\mathcal{V}$-full cover of $X$.

Our use of this term is motivated by the standard use of the term blanketed to describe those gauge functions $g$ satisfying

$$
g(r) \leq M g(r / 2)
$$

for some fixed constant $M$ and $r \geq 0$. The choice $g(r)=r^{\alpha}$ is blanketed for each $\alpha$. Applying Proposition 4.2 in the context of Examples 3.3 and 3.11 we deduce that for any Borel set $E$ in a metric space $X$,

$$
\mathcal{H}_{\alpha}(E) \leq \mathcal{P}_{\alpha}(E)
$$

for all $\alpha$ and so

$$
\operatorname{dim}_{H} E \leq \operatorname{dim}_{P} E .
$$

We now define local versions of the notions of Ahlfors regular measures and uniformly perfect metric spaces.

Definition 4.7. Let $Q \geq 0$ and let $\mu$ be a Borel measure on $X$. We say that $\mu$ satisfies local lower mass bounds with exponent $Q$ if there exists a constant $c_{0}>0$ so that the collection of closed balls $\bar{B}(x, r)$ for which

$$
\mu(\bar{B}(x, r)) \geq c_{0} r^{Q}
$$

is a $\mathcal{B}_{1}(X)$-full cover of $X$. Equivalently, there exists $c_{0}>0$ so that for each point $x \in X$ there exists $r(x)>0$ so that (4.8) holds for all $0<r \leq r(x)$.

If $\mu$ satisfies global upper mass bounds and local lower mass bounds with exponent $Q$, we call the space $(X, \mu)$ locally Ahlfors $Q$-regular.

This choice of terminology is clearly somewhat nonstandard. However, at several points in what follows (for example, in the proof of Proposition 8.9) it will be crucial that we assume global upper mass bounds on the underlying space. Furthermore, our primary application (Theorem 10.9) will be to open subsets of globally Ahlfors $Q$-regular spaces. Such a set (with metric and measure inherited from the larger space) is clearly locally Ahlfors $Q$-regular in the above sense. For the reader's convenience, we will re-emphasize this point when it is relevant.

For ease of exposition we include the following definition here, although this concept will not be used until later. 
Definition 4.9. A metric space $X$ is said to be locally uniformly perfect if there exists a constant $c_{1}>0$ so that the collection of closed balls $\bar{B}(x, r)$ in $X$ for which

$$
\operatorname{diam} \bar{B}(x, r) \geq 2 c_{1} r
$$

is a $\mathcal{B}_{1}(X)$-full cover of $X$.

For example, $X$ is locally uniformly perfect if it has no point components; note that such a space need not be uniformly perfect as there may exist sequences of balls $B_{i}=\bar{B}\left(x_{i}, r_{i}\right)$ with $r_{i}$ large for which $\operatorname{diam} B_{i} / r_{i} \rightarrow 0$.

We leave it to the reader to verify the following claim: every locally $Q$-regular space, $Q>0$, is locally uniformly perfect.

Proposition 4.10. If $\mu$ is a nontrivial Borel measure on $X$ which satisfies upper mass bounds with exponent $Q$, then $\operatorname{dim}_{H} X \geq Q$. On the other hand, if $\mu$ is locally finite (finite on bounded sets) and satisfies local lower mass bounds with exponent $Q$, then $\operatorname{dim}_{P} X \leq Q$.

Corollary 4.11. Every locally Q-regular metric measure space has Hausdorff and packing dimension equal to $Q$. If $X$ is a locally compact, separable metric space and $\mu$ is a locally $Q$-regular Borel regular measure on $X$, then $\mu, \mathcal{H}_{Q}$ and $\mathcal{P}_{Q}$ are all comparable.

Both of these results are variations of well-known facts. For example, the first sentence of Proposition 4.10 is just the familiar mass distribution principle. For Corollary 4.11] compare the remark following Definition [1.1. We include short proofs of these two facts for the convenience of the reader.

Proof of Proposition 4.10. Suppose that $\mu$ is a nontrivial measure which satisfies (1.2) for some $Q$. Let $E$ be any Borel set in $X$ and let $B_{1}, B_{2}, \ldots$ be any collection of balls covering $E$. If $r_{i}$ denotes the radius of $B_{i}$, then

$$
\mu(E) \leq \sum_{i} \mu\left(B_{i}\right) \leq C_{0} \sum_{i} r_{i}^{Q}
$$

and so

$$
\mu(E) \leq C_{0} \mathcal{H}_{Q}^{\infty}(E) \leq C_{0} \mathcal{H}_{Q}(E) .
$$

Taking $E=X$ yields $\operatorname{dim}_{H} X \geq Q$.

Now suppose that $\mu$ is a Borel measure which is finite on bounded sets and satisfies local lower mass bounds for some $Q$. Fix a bounded set $K$ in $X$. Let $\mathcal{C}$ denote the collection of balls $\bar{B}(x, r)$ with $x \in K$ and $r \leq \operatorname{diam} K$ for which (4.8) holds; $\mathcal{C}$ is a $\mathcal{B}_{1}(K)$-full collection. If $\left\{B_{1}, B_{2}, \ldots\right\}$ is any packing drawn from $\mathcal{C}$, then

$$
\sum_{i} r_{i}^{Q} \leq C \sum_{i} \mu\left(B_{i}\right) \leq C \mu(\hat{K})
$$

where $\hat{K}=\{x \in X: \operatorname{dist}(x, K) \leq \operatorname{diam} K\}$. Thus

$$
\mathcal{P}_{Q}(K) \leq V\left(\operatorname{rad}^{Q}, \mathcal{C}\right) \leq C \mu(\hat{K})<\infty,
$$

where rad denotes the radius set function: $\operatorname{rad}(\bar{B}(x, r))=r$. Thus $\operatorname{dim}_{P} K \leq Q$ for every bounded set $K$, whence $\operatorname{dim}_{P} X \leq Q$. 
Proof of Corollary 4.11. The first statement is immediate from Proposition 4.10] Assume that $\mu$ is locally $Q$-regular. We will show that there exist constants $c>0$ and $C<\infty$ so that

$$
c \mu(E) \leq \mathcal{H}_{Q}(E) \leq \mathcal{P}_{Q}(E) \leq C \mu(E)
$$

for all Borel sets $E$ in $X$. The left-hand inequality was established in (4.12) and the center inequality follows from (4.6). To prove the right-hand inequality, we first note that the locally finite Borel measure $\mu$ is outer regular:

$$
\mu(E)=\inf \{\mu(U) \mid U \supset E \text { and } U \text { is open }\}, \quad E \subset X \text { Borel, }
$$

and that the Borel measure $\mathcal{P}_{Q}$ on the locally compact, separable metric space $X$ is inner regular:

$$
\mathcal{P}_{Q}(E)=\sup \left\{\mathcal{P}_{Q}(K) \mid K \subset E \text { and } K \text { is compact }\right\}, \quad E \subset X \text { Borel. }
$$

(See for example [26, 2.2.2] or [32, Theorem 14.1].) Now for any Borel set $E$ in $X$, we choose a compact set $K$ and an open set $U$ with $K \subset E \subset U$. Since $X$ is locally compact, we may choose $\delta$ so that $B(K, \delta) \subset U$ is precompact. Reasoning as in the proof of 4.13), we find that

$$
\mathcal{P}_{Q}(K) \leq C \mu(B(K, \delta)) \leq C \mu(U) .
$$

The right-hand inequality in (4.14) now follows from (4.16), (4.15) and (4.17).

\section{A Carathéodory-type construction for line integrals}

The measures constructed in (3.2) generalize the classical Hausdorff measures $\mathcal{H}_{\alpha}, 0 \leq \alpha<\infty$. In the case $\alpha=1$, we have another natural measure defined for a rectifiable curve $\gamma: I \rightarrow X$, namely, the arc length measure $d s$ (defined in terms of the parametrization). The following construction is a natural analog of the arc length measure in this setting which makes sense even for nonrectifiable curves. As before, we fix here a real number $k \geq 1$ and a fine collection $\mathcal{V} \subset \mathcal{B}_{k}(X)$.

Definition 5.1. Let $\gamma: I \rightarrow X$ be a curve, where $I$ is an interval in $\mathbb{R}$. Let $\Lambda$ be a countable indexing set and consider a collection $\mathcal{C}=\left\{\left(I_{\lambda}, A_{\lambda}\right)\right\}_{\lambda \in \Lambda}$, where $I_{\lambda} \subset I$ is a relatively open subinterval and $A_{\lambda} \in \mathcal{V}$. (We allow here the possibility of repetitions, that is, a given quasiround set $A \in \mathcal{V}$ may appear in the collection $\mathcal{C}$ for several different values of $\lambda \in \Lambda$.) We call such a collection a parametrized (or indexed) cover of $\gamma$ if $I=\bigcup_{\lambda} I_{\lambda}$ and

$$
\gamma\left(I_{\lambda}\right) \subset A_{\lambda}
$$

for all $\lambda \in \Lambda$. If $\psi: \mathcal{V} \rightarrow[0, \infty]$, we define the $\psi$-length of $\gamma$ as follows: first, if $I$ is compact, we set

$$
\Psi \text {-length } k, \mathcal{V}(\gamma)=\lim _{\delta \rightarrow 0} \Psi \text {-length }{ }_{k, \mathcal{V}}^{\delta}(\gamma)
$$

where

$$
\Psi \text {-length }{ }_{k, \mathcal{V}}^{\delta}(\gamma)=\inf \sum_{\lambda} \psi\left(A_{\lambda}\right),
$$

the infimum being taken over all parametrized covers $\mathcal{C}=\left\{\left(I_{\lambda}, A_{\lambda}\right)\right\}$ of $\gamma$ for which each $A_{\lambda} \in \mathcal{V}$ has an outer radius $r_{2}\left(A_{\lambda}\right) \leq \delta$. For general curves $\gamma: I \rightarrow X$, we set

$$
\Psi \text {-length } k, \mathcal{V}(\gamma)=\sup _{I_{0}} \Psi \text {-length } k, \mathcal{V}\left(\gamma_{0}\right)
$$

the supremum being taken over all compact subintervals $I_{0} \subset I$. 
As always, we abbreviate $\Psi$-length ${ }_{k, \mathcal{B}_{k}(X)}=\Psi$-length ${ }_{k}$.

Remarks 5.4. (1) Definition (5.2) makes sense for all curves (regardless of whether the parametrizing interval is compact or not) and one may ask if the two definitions (5.2) and (5.3) agree in general. Although this seems plausible, we have been unable to give a proof for completely general choices of the set function $\psi$. The choice of (5.3) for the definition of the $\psi$-length of a curve with noncompact parametrizing interval is necessary for the proof of Proposition 9.1.

(2) The quantity $\Psi$-length $k, \mathcal{V}(\gamma)$ does not depend on the parametrization of $\gamma$. Indeed, if $\gamma^{\prime}: I^{\prime} \rightarrow X$ is a reparametrization of $\gamma$, we can always find corresponding intervals $\left\{I_{\lambda}^{\prime}\right\}$ for which $I^{\prime}=\bigcup_{\lambda} I_{\lambda}^{\prime}$ and $\gamma\left(I_{\lambda}\right)=\gamma^{\prime}\left(I_{\lambda}^{\prime}\right)$. For this reason, when the curve $\gamma$ is rectifiable, we will always assume it is parametrized by arc length.

(3) We always have

$$
\Psi \text {-length } k, \mathcal{V}(\gamma) \geq \Psi_{k, \mathcal{V}}(|\gamma|),
$$

which generalizes the fact that length $(\gamma) \geq \mathcal{H}_{1}(|\gamma|)$. This is immediate since any parametrized cover is (in particular) a cover of the image set.

Further evidence for the claim that the $\psi$-length generalizes the classical notion of length is provided by the following lemma:

Lemma 5.5. Let $X$ be a metric space and let $\psi: \mathcal{V} \rightarrow[0, \infty]$ be given by $\psi(A)=$ $\operatorname{diam} A$. If $\gamma: I \rightarrow X$ is any curve, then

$$
\operatorname{length}(\gamma) \leq \Psi \text {-length } k, \mathcal{v}(\gamma) \leq C \text { length }(\gamma)
$$

where $C$ is a constant depending only on $k$.

When $\mathcal{V}=\mathcal{B}_{k}(X)$, we can improve (5.6) to length $(\gamma)=\Psi$-length ${ }_{k}(\gamma)$ (see Lemma 3.18 of [69]). The reason why (5.6) is weaker is that the proof applies to arbitrary fine collections $\mathcal{V}$.

Proof. It suffices to consider the case when the parametrizing interval $I=[a, b]$ is compact.

We prove the right-hand inequality first. If $\gamma$ is not rectifiable, there is nothing to prove. If $\gamma$ is rectifiable and $L=\operatorname{length}(\gamma)$, then we may assume that $\gamma:[0, L] \rightarrow X$ is parametrized by arc length. Let $\delta>0$ be chosen sufficiently small (the exact choice will be given later). For each $x \in|\gamma|$, choose $r_{1 x}$ and $r_{2 x}$ with $r_{1 x} \leq r_{2 x}<$ $k r_{1 x}$ and $r_{2 x} \leq \delta$ and (recalling Definition 2.1) $r_{2 x}-r_{1 x} \geq c(k) r_{1 x}>0$, where $c(k)=(k-1) / 2$.

Assume that $\delta \leq \operatorname{diam}|\gamma| /(4 k)$. Then $|\gamma| \not \subset \bar{B}\left(x, r_{1 x}\right)$ and so $\operatorname{diam} \bar{B}\left(x, r_{1 x}\right) \geq$ $r_{1 x}$. But then

$$
\operatorname{diam} \bar{B}\left(x, r_{2 x}\right) \leq 2 r_{2 x}<2 k r_{1 x} \leq 2 k \operatorname{diam} \bar{B}\left(x, r_{1 x}\right) \leq \operatorname{diam}|\gamma|
$$

and so $|\gamma| \not \subset \bar{B}\left(x, r_{2 x}\right)$.

The sets $\left\{B\left(x, r_{1 x}\right): x \in|\gamma|\right\}$ form an open cover of the compact set $|\gamma|$. Choose a finite subcover $B_{11}, B_{12}, \ldots, B_{1 M}$, where, for each $j=1,2, \ldots, M$ and $i=1,2$ we set $B_{i j}=B\left(x_{j}, r_{i x_{j}}\right)$.

For each $j=1,2, \ldots, M$, let $\left\{\overline{I_{j l}}: l=1,2, \ldots L_{j}\right\}$ be a maximal collection of maximal disjoint closed intervals in $[0, L]$ for which $\gamma\left(\overline{I_{j l}}\right) \subset \bar{B}_{2 j}$ and $\gamma\left(\overline{I_{j l}}\right) \cap \bar{B}_{1 j} \neq$ $\emptyset$. We will show momentarily that in this situation $L_{j}$ must be finite so that the 
existence of such a maximal collection is not an issue 8 Denote by $I_{j l}$ the open interval which is the interior of $\overline{I_{j l}}$ in $\mathbb{R}$. Since $\gamma\left(\overline{I_{j l}}\right)$ meets $\partial \bar{B}_{2 j}$,

$$
\operatorname{length}\left(\left.\gamma\right|_{I_{j l}}\right) \geq r_{2 x_{j}}-r_{1 x_{j}} \geq c(k) r_{1 x_{j}}>0
$$

for each $l=1,2, \ldots, L_{j}$ and $j=1,2, \ldots, M$. Hence

$$
L_{j} \cdot c(k) r_{1 x_{j}} \leq \sum_{l=1}^{L_{j}} \operatorname{length}\left(\left.\gamma\right|_{I_{j l}}\right) \leq L
$$

and so $L_{j}$ is finite. The maximality of the collections $\left\{\overline{I_{j l}}\right\}, j=1,2, \ldots, M$, together with the fact that $\left\{B_{1 j}\right\}$ covers $|\gamma|$, implies that

$$
[0, L] \subset \bigcup_{j} \bigcup_{l=1}^{L_{j}} I_{j l} .
$$

By throwing out some of the intervals $I_{j l}$ if necessary, we may assume that each point in $[0, L]$ lies in at most two intervals. The collection of pairs $\left\{\left(I_{j l}, \bar{B}_{2 j}\right): 1 \leq\right.$ $\left.j \leq M, 1 \leq l \leq L_{j}\right\}$ forms a parameterized cover of $\gamma$ with $r_{2 j} \leq \delta$ for each $j$. Hence

$$
\begin{aligned}
\Psi-\operatorname{length}_{k, \mathcal{V}}^{\delta}(\gamma) & \leq \sum_{j=1}^{M} L_{j} \operatorname{diam} \bar{B}_{2 j}<2 k \sum_{j=1}^{M} L_{j} r_{1 x_{j}} \\
& \leq \frac{2 k}{c(k)} \sum_{j=1}^{M} \sum_{l=1}^{L_{j}} \operatorname{length}\left(\left.\gamma\right|_{I_{j l}}\right) \leq C \cdot L
\end{aligned}
$$

where $C=4 k / c(k)=8 k /(k-1)$. Letting $\delta \rightarrow 0$ completes the proof of the right-hand inequality.

Next, we show the left-hand inequality. Assume that $\gamma: I \rightarrow X$ and choose a partition $\pi=\left\{a=t_{0}<t_{1}<\cdots<t_{m}=b\right\}$ of $I$. We may assume without loss of generality that $\gamma\left(t_{j}\right) \neq \gamma\left(t_{j-1}\right)$ for $j=1,2, \ldots, m$. Let

$$
\delta_{0}=\min \left\{\left|\gamma\left(t_{j}\right)-\gamma\left(t_{j-1}\right)\right|: j=1,2, \ldots, m\right\} .
$$

If $\delta<\frac{1}{2} \delta_{0}$ and $\left\{\left(J_{\lambda}, A_{\lambda}\right)\right\}_{\lambda \in \Lambda}$ is an arbitrary parametrized cover of $\gamma$ by sets $A_{\lambda} \in \mathcal{V}$ with outer radius $r_{2}\left(A_{\lambda}\right) \leq \delta$ for all $\lambda$, then each of the open intervals $J_{\lambda}$ contains at most one of the $t_{j}$. Since $I$ is assumed to be compact, there is a minimal finite subcollection $\Lambda^{\prime} \subset \Lambda$ with $I \subset \bigcup_{\lambda \in \Lambda^{\prime}} J_{\lambda}$. Using the triangle inequality, we estimate

$$
\sum_{j=1}^{m}\left|\gamma\left(t_{j}\right)-\gamma\left(t_{j-1}\right)\right| \leq \sum_{\lambda \in \Lambda^{\prime}} \operatorname{diam} A_{\lambda}+(m+1) \delta
$$

where the second term on the right-hand side is necessary to account for the sets covering the points $\gamma\left(t_{0}\right), \gamma\left(t_{1}\right), \ldots, \gamma\left(t_{m}\right)$, where we may have to allow a given quasiround set $A_{\lambda}$ to be counted twice. Therefore

$$
\sum_{j=1}^{m}\left|\gamma\left(t_{j}\right)-\gamma\left(t_{j-1}\right)\right| \leq \Psi \text {-length }{ }_{k, \mathcal{V}}^{\delta}(\gamma)+(m+1) \delta .
$$

Letting $\delta \rightarrow 0$ and taking the supremum over partitions $\pi$ finishes the proof.

A generalization of this argument will be crucial in our proof of Proposition 8.9

\footnotetext{
${ }^{8}$ Note that at least one such interval must always exist since $|\gamma|$ meets $B_{1 j}$ but is not contained in $B_{2 j}$.
} 


\section{PART 2. Generalized MOdUli OF CURVE FAMILIES AND THE MAIN THEOREM}

In the following sections, we introduce and make use of the fundamental tool of this paper: the generalized modulus of a family of curves. This concept was originally introduced by Pansu [51], 52] in his study of Lipschitz and quasiconformal maps on the boundaries of rank one symmetric spaces. Pansu's definition was used in [69] to study quasisymmetry and geometric quasiconformality in general Ahlfors regular spaces. Corollary 6.7 generalizes the main result which we established in 69] to locally quasisymmetric maps in locally compact, locally Ahlfors regular spaces. For this purpose, we introduce a generalized modulus based on packingtype measures. Generalized moduli based on Hausdorff-type measures were used in 51, 52] and 69.

Section 6 contains a brief descriptive overview of the principal results of this part of the paper. The generalized modulus will be defined in section 7 and the proof of the main theorem will begin in section 8 .

\section{Preliminary Remarks}

We recall the metric definition for quasiconformality. Assume that $X$ and $Y$ are metric spaces (of cardinality at least two) and let $f: X \rightarrow Y$ be a homeomorphism. For $x \in X$ and $r>0$, we define the maximal and minimal stretchings:

$$
\begin{aligned}
L_{f}(x, r) & =\sup \{|f x-f y|: y \in \bar{B}(x, r)\}, \\
l_{f}(x, r) & =\inf \{|f x-f z|: z \in X \backslash B(x, r)\} .
\end{aligned}
$$

Then $f$ is called metrically $H$-quasiconformal, $1 \leq H<\infty$, if

$$
\limsup _{r \rightarrow 0} \frac{L_{f}(x, r)}{l_{f}(x, r)} \leq H
$$

for each $x \in X 9$ We can restate this condition as follows: at each point $x \in X$, $f$ takes infinitesimally small balls centered at $x$ onto elements of $\mathcal{B}_{l^{\prime}}(Y)$ centered at $f(x)$, where $l^{\prime}$ is any value strictly greater than $H$. In the language of section 2, the collection of balls in $X$ for which this condition holds is a $\mathcal{B}_{1}(X)$-full cover of $X$.

In the case when $X=Y=\mathbb{R}^{n}, n \geq 2$, or more generally when $X$ is $Q$-regular and Loewner (Definition 10.2) and $Y$ is $Q$-regular and LLC, any map $f$ which satisfies (6.2) is necessarily locally quasisymmetric [38]. In fact, it suffices in $\mathbb{R}^{n}$ to assume a weaker condition, where limsup in (6.2) is replaced by liminf, see [37. The proof of this fact relies on the Besicovitch covering theorem, which is a very strong covering theorem holding in $\mathbb{R}^{n}$. Although this covering theorem does not hold in its strongest form in the abstract setting, a slightly weaker form (Lemma 8.4) does hold. This fact was used by Balogh and Koskela [3] to show that if $X$

\footnotetext{
${ }^{9}$ In Euclidean space, quasiconformal maps are usually defined in terms of the related quantities

$$
\begin{aligned}
L_{f}^{*}(x, r) & =\sup \{|f x-f y|: y \in S(x, r)\}, \\
l_{f}^{*}(x, r) & =\inf \{|f x-f z|: z \in S(x, r)\},
\end{aligned}
$$
}

but this choice may not work in an arbitrary metric space where, for example, a given sphere $S(x, r)$ may in fact be empty. If the target space is locally $C$-LLC, then

$$
B\left(f x, l_{f}^{*}(x, r) / C\right) \subset f B(x, r) \subset f \bar{B}(x, r) \subset \bar{B}\left(f x, C L_{f}^{*}(x, r)\right)
$$

for each $x \in X$ and sufficiently small $r>0$, which implies that $l_{f}^{*}(x, r) / C \leq l_{f}(x, r)$ and $L_{f}(x, r) \leq$ $C L_{f}^{*}(x, r)$. We leave the proof of this fact to the reader. 
and $Y$ are as above, then a homeomorphism $f: X \rightarrow Y$ is locally quasisymmetric provided that there exist fixed constants $t>1$ and $H<\infty$ so that

$$
\liminf _{r \rightarrow 0} \sup _{r \leq s \leq t r} \frac{L_{f}(x, s)}{l_{f}(x, s)} \leq H
$$

for all $x \in X$.

In [69, the author used (Hausdorff-type) generalized moduli to show that quasisymmetric maps $f: X \rightarrow Y$ between locally compact Ahlfors $Q$-regular spaces, $Q>1$, satisfy the geometric definition of quasiconformality. Here, we use packingtype generalized moduli to prove the following result:

Theorem 6.4. Let $X$ and $Y$ be locally compact metric measure spaces which are locally Ahlfors $Q$-regular for some $Q>1$. Let $f: X \rightarrow Y$ be a homeomorphism satisfying the following condition: there exist constants $H<\infty$ and $t>1$ so that

$$
\liminf _{r \rightarrow 0} \frac{L_{f}(x, t r)}{l_{f}(x, r)} \leq H
$$

for all $x \in X$. Then there exists $K<\infty$ depending only on $t, H$ and the regularity data of $X$ and $Y$ so that

$$
\operatorname{Mod}_{Q} \Gamma \leq K \operatorname{Mod}_{Q} f \Gamma
$$

for every curve family $\Gamma$ in $X$.

Recall (Definition 4.7) that a metric measure space $(X, \mu)$ is said to be locally Ahlfors $Q$-regular if the measure $\mu$ satisfies global upper mass bounds and local lower mass bounds with exponent $Q$.

It is easy to see that (6.5) is weaker than (6.3). Indeed, for fixed $x$, the quantity $L_{f}(x, r)$, resp. $l_{f}(x, r)$ is an increasing, resp. decreasing function of $r$. The conclusion of Theorem 6.4 is different from that of the main result of [3], however. Moreover, our results apply to arbitrary open subsets of Ahlfors regular spaces. One drawback is that (6.5) is not implied by the classical quasiconformality condition (6.2), in contrast to (6.3). Even if we replace the lim inf in (6.5) by a limsup, our techniques suffice to establish the conclusion only in the case $t>1$. I do not know if it is possible in general to take $t=1$ in Theorem 6.4

It is trivial to see that locally quasisymmetric maps satisfy (6.5) for any $t>1$; in fact, they satisfy the stronger condition where lim inf is replaced by lim sup (see Lemma 9.3). Since the inverse of a locally quasisymmetric map is again locally quasisymmetric, Theorem 6.4 has the following corollary:

Corollary 6.7. Let $X$ and $Y$ be locally compact metric measure spaces which are locally Ahlfors $Q$-regular (Definition 4.7) for some $Q>1$ and locally $C_{1}-L L C$ for some $C_{1} \geq 1$. Let $f: X \rightarrow Y$ be a locally $\eta$-quasisymmetric homeomorphism. Then $f$ is geometrically $K$-quasiconformal for some constant $K$ depending only on $\eta, C_{1}$, and the regularity data of $X$ and $Y$.

Note that in Theorem 6.4 and Corollary 6.7 the Loewner condition is not assumed. However, in order to prove the full result stated in the introduction, we must make this additional assumption. In section [10, we will prove that any map $f$ from an open subset $U$ of a Loewner space onto an open subset $U^{\prime}$ of an LLC space, which satisfies (6.5) at each point of $x \in U$ for fixed constants $t>1$ and $H<\infty$, is locally quasisymmetric and also geometrically quasiconformal. 
In the language of section 2, condition (6.5) can be reformulated as follows: for each $l^{\prime}>H$ the collection of rings $(B, t B)$ centered at $x \in X$ whose image is an $l^{\prime}$-quasiring (see below) in $Y$ centered at $f(x)$ is a fine cover of $X$. This gives some indication of how the machinery developed in the previous sections will be used in the proof of Theorem 6.4

More precisely, the basic structure of the proof of Theorem 6.4 is as follows. We first show that in locally Ahlfors regular spaces the generalized and classical moduli of a curve family agree up to a bounded factor, which may depend on certain data associated with the generalized modulus and on the regularity data of the underlying space but is independent of the curve family. We then show that starting from a homeomorphism $f: X \rightarrow Y$ which satisfies (6.5), we can choose appropriate data (depending on the map $f$ ) so that the generalized modulus of any curve family is not decreased under the map $f$. Theorem 6.4 follows. The details of this argument will be given in sections 8 and 9. In section 10 we define the Loewner condition and prove the main result (Theorem 10.9) mentioned earlier. In section [1] we briefly describe several recently constructed examples of Ahlfors regular Loewner spaces.

\section{The Generalized Modulus of A CURVE FAMily}

Let $X$ be a metric space. In Definition 4.4 we defined the notion of a locally blanketed set function on metric balls in $X$. In terms of the classes $\mathcal{B}_{k}(X), k \geq 1$, this corresponds to the case $k=1$. We next extend Definition 4.4 to all $k$.

Definition 7.1. Fix $l>1$. Let $A, \tilde{A} \subset X$ be closed sets with $A \subset \tilde{A}$. We call the pair $(A, \tilde{A})$ an l-quasiring if there exist $x \in X$ and $0<f_{1} \leq r_{2}<l r_{1}<\infty$ so that

$$
\bar{B}\left(x, r_{1}\right) \subset A \subset \tilde{A} \subset \bar{B}\left(x, r_{2}\right) .
$$

Definition 7.3. Let $l>k \geq 1$ and let $\mathcal{V}$ and $\tilde{\mathcal{V}}$ be two fine collections in $\mathcal{B}_{k}(X)$. We call $(\mathcal{V}, \tilde{\mathcal{V}})$ an $l$-admissible pair if the following condition is satisfied: there exists a map $A \mapsto \tilde{A}$ from $\mathcal{V}$ to $\tilde{\mathcal{V}}$ so that the pair $(A, \tilde{A})$ is an $l$-quasiring for each $A \in \mathcal{V}$.

For example, when $\mathcal{V}=\tilde{\mathcal{V}}=\mathcal{B}_{1}(X)$ is the collection of closed balls in $X$ and $t>1$, we will take

$$
\widetilde{B(x, r)}=\bar{B}(x, t r)
$$

clearly the pair $(\bar{B}(x, r), \bar{B}(x, t r))$ is an $l$-quasiring for each $l>t$.

More generally, if $\mathcal{V}=\tilde{\mathcal{V}}=\mathcal{B}_{k}(X)$ for some $k \geq 1$ and $t>1$, we will take $\tilde{A}=\bar{B}\left(x, t r_{2}\right)$ for $A \in \mathcal{V}$, where the triple $x, r_{1}, r_{2}$ is chosen as in (2.2). Note that the pair $(A, \tilde{A})$ is an $l$-quasiring for each $l>t k$.

Definition 7.4. Let $(\mathcal{V}, \tilde{\mathcal{V}})$ be an $l$-admissible pair and let $M<\infty$. We call a set function $\psi: \mathcal{V} \cup \tilde{\mathcal{V}} \rightarrow[0, \infty]$ locally $M$-blanketed if the collection of quasiround sets $A \in \mathcal{V}$ which satisfy

$$
\psi(\tilde{A}) \leq M \psi(A)
$$

is a $\mathcal{V}$-full cover of $X$.

Example 7.6. Let $X$ be a locally uniformly perfect metric space with constant $c_{1}$ and let $(\mathcal{V}, \tilde{\mathcal{V}})$ be an $l$-admissible pair of fine collections in $\mathcal{B}_{k}(X), l>k \geq 1$. Let $\psi$ 
be a set function satisfying

$$
\psi(A)=g(\operatorname{diam} A),
$$

where $g$ is a blanketed gauge function, i.e., $g$ is a continuous increasing map of $[0, \infty)$ to itself for which $g(r) \leq M g(r / 2)$ for all $r \geq 0$ and some $M<\infty$. Then $\psi$ is locally $M^{\prime}$-blanketed, where $M^{\prime}=M^{\prime}\left(M, l, c_{1}\right)$.

We have now described all of the ingredients necessary to define the (packingtype) generalized modulus of a curve family.

Definition 7.7. Let $X$ be a metric space. Fix values $l>k \geq 1, M<\infty$, and fine collections $\mathcal{V}, \tilde{\mathcal{V}} \subset \mathcal{B}_{k}(X)$ for which the pair $(\mathcal{V}, \tilde{\mathcal{V}})$ is $l$-admissible. Let $\Gamma$ be a family of curves in $X$.

The (packing-type) generalized p-modulus of $\Gamma$ with data $\sigma=(k, l, M)$ is the number

$$
\bmod _{p, \sigma}^{\bullet} \Gamma=\inf \Phi_{p, k, \mathcal{V}}^{\bullet}(X),
$$

where the infimum is taken over all locally $M$-blanketed functions $\varphi: \mathcal{V} \cup \tilde{\mathcal{V}} \rightarrow[0, \infty]$ satisfying $\Phi$-length $k, \tilde{\mathcal{V}}(\gamma) \geq 1$ for all $\gamma \in \Gamma$. Here we denote by $\Phi_{p, k, \mathcal{V}}^{\bullet}$ the packing measure constructed using the family $\mathcal{V}$ and the function $\varphi^{p}: \mathcal{V} \rightarrow[0, \infty]$ (see Definition 3.6.

Remarks 7.8. (1) In [69] generalized moduli defined in terms of the Hausdorff-type measures $\Phi_{p, k, \mathcal{V}}$ were used to study globally defined quasisymmetric maps in Ahlfors regular spaces. Definition 7.7 appears to be the appropriate concept to use to study locally quasisymmetric mappings in locally Ahlfors regular spaces. This setting is crucial for our main result (Theorem 6.4) as well as for the application in section 13

(2) It would be more precise to include the fine collections $\mathcal{V}$ and $\tilde{\mathcal{V}}$ in the data $\sigma=(k, l, M)$. However, as we shall soon see, these parameters play no quantitative role in the estimates and so for simplicity we do not include them in the notation.

The following result for the generalized modulus corresponds to Proposition 1.4(i)-(iv) for the classical modulus. In contrast to the classical modulus, we are only able to show that the generalized modulus is finitely subadditive. In other words, $\bmod _{p, \sigma}^{\bullet}(\cdot)$ is an outer content on the collection of all curves. The Hausdorfftype generalized modulus considered in [69] does not satisfy this finite subadditivity condition (see Remark 3.4).

Proposition 7.9. Let $p>0$ and let $\sigma=(k, l, M), \mathcal{V}$ and $\tilde{\mathcal{V}}$ be as above. Then

(i) $\bmod _{p, \sigma}^{\bullet} \emptyset=0$;

(ii) if $\Gamma \subset \Gamma^{\prime}$, then $\bmod _{p, \sigma}^{\bullet} \Gamma \leq \bmod _{p, \sigma}^{\bullet} \Gamma^{\prime}$;

(iii) for any finite collection $\Gamma_{1}, \Gamma_{2}, \ldots, \Gamma_{N}$ of curve families,

$$
\bmod _{p, \sigma}^{\bullet} \bigcup_{i=1}^{N} \Gamma_{i} \leq \sum_{i=1}^{N} \bmod _{p, \sigma}^{\bullet} \Gamma_{i}
$$

(iv) $\bmod _{p, \sigma}^{\bullet} \Gamma \leq \bmod _{p, \sigma}^{\bullet} \Gamma^{\prime}$ if $\Gamma$ is minorized by $\Gamma^{\prime}$.

By analogy with the classical modulus, we say that a property $\mathcal{P}$ of curves holds for $\bmod _{p}^{\bullet}$-almost every curve if the collection $\Gamma$ of curves for which $\mathcal{P}$ fails to hold has generalized $p$-modulus zero, which (in light of the fact that the generalized modulus is not countably subadditive) we take to mean the following: $\Gamma$ can be 
written as a countable union $\Gamma=\bigcup_{n=1}^{\infty} \Gamma_{n}$ so that the generalized $p$-modulus of $\Gamma_{n}$ is equal to zero for each $n$ and each choice of the data $\sigma=(k, l, M), k \geq 1, l>k$ and some (equivalently, all sufficiently large) $M \geq 1$.

The only part of Proposition [7.9 which is not completely trivial is (iii). To prove this, let $\varphi_{1}, \ldots, \varphi_{N}$ be locally $M$-blanketed functions on $\mathcal{V} \cup \tilde{\mathcal{V}}$ for which $\Phi_{i}$-length $_{k, \tilde{\mathcal{V}}}(\gamma) \geq 1$ for each $\gamma \in \Gamma_{i}, i=1, \ldots, N$. Define $\varphi: \mathcal{V} \cup \tilde{\mathcal{V}} \rightarrow[0, \infty]$ by

$$
\varphi(A)=\left(\sum_{i=1}^{N} \varphi_{i}(A)^{p}\right)^{1 / p}
$$

Then $\varphi$ is a locally $M$-blanketed function (since the intersection of a finite number of $\mathcal{V}$-full covers is again a $\mathcal{V}$-full cover). Since $\varphi \geq \varphi_{i}$ for each $i, \Phi$-length $k, \tilde{\mathcal{V}}(\gamma) \geq 1$ for each $\gamma \in \Gamma$. Finally, the measure $\Phi_{p, k, \mathcal{V}}^{\bullet}$ is controlled by the sum of the measures $\left(\Phi_{i}\right)_{p, k, \mathcal{V}}^{\bullet}, i=1, \ldots, N$. Thus

$$
\bmod _{p, \sigma}^{\bullet} \Gamma \leq \Phi_{p, k, \mathcal{V}}^{\bullet}(X) \leq \sum_{i=1}^{N}\left(\Phi_{i}\right)_{p, k, \mathcal{V}}^{\bullet}(X)
$$

and (7.10) follows by taking the infimum over all such $N$-tuples $\varphi_{1}, \ldots, \varphi_{N}$.

\section{Relations Between the ClassicAl AND Generalized Moduli}

We prove two theorems relating the classical and generalized moduli. Taken together, they yield the following result: in each locally compact, locally Ahlfors $Q$-regular space, $Q>1$, for each choice of $l>t>1$ and $M \in\left[M_{0}, \infty\right)$ there exists $C \geq 1$ (depending only on $t, M$ and the regularity data of the space) so that

$$
\frac{1}{C} \bmod _{Q,(1, l, M)}^{\bullet} \Gamma \leq \operatorname{Mod}_{Q} \Gamma \leq C \bmod _{Q,(1, l, M)}^{\bullet} \Gamma
$$

for all curve families $\Gamma$ and all $l$-admissible pairs $(\mathcal{V}, t \mathcal{V})$ of fine collections of balls. (Here $M_{0} \geq 1$ is a fixed constant which depends only on $l$ and the regularity data of the space.)

Recall that the classical modulus $\operatorname{Mod}_{Q} \Gamma$ depends only on the locally rectifiable curves in $\Gamma$, while the generalized $\operatorname{modulus} \bmod _{Q, \sigma}^{\bullet} \Gamma$ depends a priori on all of the elements of $\Gamma$. It follows from (8.1) that $\bmod _{Q, \sigma}^{\bullet}$-a.e. curve in $X$ is locally rectifiable, which is not at all obvious from the definition. A consequence of this is given in section [12,

Proposition 8.2. Let $(X, \mu)$ be a locally Ahlfors $Q$-regular metric measure space, $Q>1$. Let $l>t>1$ and $M<\infty$. Then there exists $K_{1} \geq 1$ depending only on $t$, $M$ and the regularity data $Q, c_{0}$ and $C_{0}$, so that

$$
\operatorname{Mod}_{Q} \Gamma \leq K_{1} \bmod _{Q,(1, l, M)}^{\bullet} \Gamma
$$

for all curve families $\Gamma$ and all fine collections $\mathcal{V} \subset \mathcal{B}_{1}(X)$, where $\tilde{\mathcal{V}}=t \mathcal{V}$.

The proof of Proposition 8.2 follows that of Proposition 4.5 of 69, which in turn was based on Lemma 2.4 of [37]. Definition (8.8) "reassembles" an admissible metric $\rho$ for the classical modulus from a set function $\varphi$ on $\mathcal{V} \cup t \mathcal{V}$ which is admissible for the generalized modulus.

Before beginning the proof, we cite two lemmas. The first, of Besicovitch type, can be proved as in [3] Lemma 3.2]; we leave the (straightforward) modifications to the reader. For the second, compare [8, Lemma 4.2]; the proof makes use of Hölder's 
inequality and the boundedness of a maximal operator of Hardy-Littlewood type on the dual of $L^{p}(X, \mu)$. For the reader's convenience, we briefly sketch this argument in an appendix.

Lemma 8.4. Let $(X, \mu)$ be a locally $Q$-regular space and let $t>1$. Then there exists $N=N\left(t, Q, c_{0}, C_{0}\right)$ so that any fine collection $\mathcal{C} \subset \mathcal{B}_{1}(X)$ with uniformly bounded radii admits a family of subcollections $\mathcal{C}_{1}, \ldots, \mathcal{C}_{N}$ with the following properties:

(i) for each $j$, the collection $\mathcal{C}_{j}$ consists of countably many pairwise disjoint balls;

(ii) the set consisting of the centers of the balls in $\mathcal{C}$ is covered by $\bigcup_{j=1}^{N} t \mathcal{C}_{j}$.

Lemma 8.5. Let $X$ be a metric space and let $\mu$ be a locally doubling measure on $X$ with doubling constant $C_{\mu}$. Let $t>1$ and let $1 \leq p<\infty$. Then there exists a $\mathcal{B}_{1}(X)$-full collection $\widehat{\mathcal{C}}(t)$ of balls, depending on $t$ and the doubling constant $C_{\mu}$, and there exists $A=A\left(t, p, C_{\mu}\right)$, so that

$$
\int_{X}\left(\sum_{i} a_{i} \chi_{t B_{i}}\right)^{p} d \mu \leq A \int_{X}\left(\sum_{i} a_{i} \chi_{B_{i}}\right)^{p} d \mu
$$

whenever $B_{1}, B_{2}, \ldots$ are elements of $\widehat{\mathcal{C}}(t)$ and $a_{1}, a_{2}, \ldots$ are nonnegative real numbers.

Here a measure $\mu$ is said to be a locally doubling measure on a metric space $X$ (with doubling constant $C_{\mu}$ ) if the collection of closed balls $B$ for which

$$
\mu(2 B) \leq C_{\mu} \mu(B)
$$

is a $\mathcal{B}_{1}(X)$-full collection. A locally $Q$-regular measure (with regularity data $c_{0}>0$ and $\left.C_{0}<\infty\right)$ is locally doubling with doubling constant $C_{\mu}=C_{\mu}\left(Q, c_{0}, C_{0}\right)$.

Proof of Proposition 8.2. Let $\varphi: \mathcal{V} \cup t \mathcal{V} \rightarrow[0, \infty]$ be a locally $M$-blanketed function which satisfies the admissibility condition $\Phi$-length ${ }_{1, t} \mathcal{V}(\gamma) \geq 1$ for each $\gamma \in \Gamma$. Let $\Gamma_{n}$ consist of all those curves $\gamma: I \rightarrow X$ in $\Gamma$ for which there exists a compact subinterval $I_{0} \subset I$ so that the set $\left|\gamma_{0}\right|$ has diameter at least $1 / n$ and

$$
\Phi \text {-length } \operatorname{siv}_{1, t \mathcal{V}}^{1 / 2 n}\left(\gamma_{0}\right) \geq \frac{1}{2}
$$

(Recall that $\gamma_{0}$ denotes the restriction of the curve $\gamma$ to $I_{0}$.) Since $\Gamma=\bigcup_{n} \Gamma_{n}$ and $Q>1$, it suffices by Proposition 1.4(viii) to show that

$$
\operatorname{Mod}_{Q} \Gamma_{n} \leq C \Phi_{Q, 1, \mathcal{V}}^{\bullet}(X)
$$

where the constant $C$ is independent of $n$.

Let $\mathcal{C}$ be a $\mathcal{V}$-full cover of $X$. Without loss of generality, we may assume that each ball $B=\bar{B}(x, r)$ in $\mathcal{C}$ satisfies $r \leq 1 /(4 n t)$ and that $\varphi(t B) \leq M \varphi(B)$. Moreover, we may assume that $\mathcal{C}$ is contained in the collection $\widehat{\mathcal{C}}(t)$ of Lemma 8.5. Since $\mathcal{C}$ is a fine collection, we may choose $\mathcal{C}_{1}, \ldots, \mathcal{C}_{N} \subset \mathcal{C}$ so that Lemma 8.4 holds. Let $\mathcal{C}^{*}:=\bigcup_{j=1}^{N} \mathcal{C}_{j}$. Then $t \mathcal{C}^{*}$ is a cover of $X$.

For any $\gamma \in \Gamma_{n}$ and $B \in \mathcal{C}$, we have $\left|\gamma_{0}\right| \not \subset t B$. Hence length $\left(\left.\gamma_{0}\right|_{J}\right) \geq(t-1) r$ for any open interval $J \subset I_{0}$ such that $\gamma(J) \cap B \neq \emptyset$ and $\overline{\gamma(J)} \cap \partial(t B) \neq \emptyset$.

Define a function $\rho: X \rightarrow[0, \infty]$ by

$$
\rho(x)=\frac{2}{t-1} \cdot \sum_{\substack{B \in \mathcal{C}^{*} \\ B=\bar{B}(x, r)}} \frac{\varphi(t B)}{r} \chi_{t B}(x) .
$$


Let $\gamma \in \Gamma_{n}$ be a locally rectifiable curve and let $\gamma_{0}$ be the associated compact subcurve. For each $B \in \mathcal{C}^{*}$ which meets $\left|\gamma_{0}\right|$, let $\Lambda_{B}$ denote the collection of all maximal open subintervals $J$ in $I_{0}$ so that $\gamma(J) \subset t B$ and $\gamma(J)$ meets $B$. Our index set $\Lambda$ is defined to be the disjoint union of the collections $\Lambda_{B}$. To each $\lambda(=J)$ in $\Lambda_{B}$, we associate the pair $\left(I_{\lambda}, B_{\lambda}\right)$, where $I_{\lambda}=J$ and $B_{\lambda}=t B$. The collection $\left\{\left(I_{\lambda}, B_{\lambda}\right): \lambda \in \Lambda\right\}$ is a parametrized cover of $\gamma_{0}$ with balls in $t \mathcal{V}$ with radii at most $1 / 2 n$. Hence (8.7) implies that $2 \sum_{\lambda} \varphi\left(B_{\lambda}\right) \geq 1$ and so

$$
\begin{aligned}
\int_{\gamma} \rho d s & \geq \int_{\gamma_{0}} \rho d s=\frac{2}{t-1} \cdot \sum_{\substack{B \in \mathcal{C}^{*} \\
B=\bar{B}(x, r)}} \frac{\varphi(t B)}{r} \operatorname{length}\left(\gamma_{0} \cap t B\right) \\
& \geq \frac{2}{t-1} \cdot \sum_{B}^{\prime} \frac{\varphi(t B)}{r} \operatorname{length}\left(\gamma_{0} \cap t B\right)
\end{aligned}
$$

(where the second sum is over all balls $B$ meeting $\left|\gamma_{0}\right|$ )

$$
\geq \frac{2}{t-1} \cdot \sum_{B}^{\prime} \frac{\varphi(t B)}{r} \sum_{J \in \Lambda_{B}} \text { length }\left(\left.\gamma_{0}\right|_{J}\right) \geq 2 \sum_{\lambda \in \Lambda} \varphi\left(B_{\lambda}\right) \geq 1 .
$$

Thus $\rho$ is an admissible metric for $\Gamma_{n}$. Setting $a_{B}=\varphi(t B) / r$ for $B=\bar{B}(x, r) \in \mathcal{C}^{*}$, we have

$$
\operatorname{Mod}_{Q} \Gamma_{n} \leq \int_{X} \rho^{Q} d \mu \leq C(t, Q) \int_{X}\left(\sum_{j=1}^{N} \sum_{B \in \mathcal{C}_{j}} a_{B} \chi_{t B}\right)^{Q} d \mu .
$$

Using Hölder's inequality and (8.6), we find that

$$
\begin{aligned}
\operatorname{Mod}_{Q} \Gamma_{n} & \leq C(t, Q, N) \sum_{j=1}^{N} \int_{X}\left(\sum_{B \in \mathcal{C}_{j}} a_{B} \chi_{t B}\right)^{Q} d \mu \\
& \leq C(t, Q, N, A) \sum_{j=1}^{N} \int_{X}\left(\sum_{B \in \mathcal{C}_{j}} a_{B} \chi_{B}\right)^{Q} d \mu
\end{aligned}
$$

where $A$ denotes the constant in (8.6). Since the elements of $\mathcal{C}_{j}$ are pairwise disjoint, we have

$$
\begin{aligned}
\operatorname{Mod}_{Q} \Gamma_{n} & \leq C(t, Q, N, A) \sum_{j=1}^{N} \sum_{B \in \mathcal{C}_{j}} a_{B}^{Q} \mu(B) \\
& \leq C\left(t, Q, N, A, C_{0}\right) \sum_{j=1}^{N} \sum_{B \in \mathcal{C}_{j}} \varphi(t B)^{Q} .
\end{aligned}
$$

Here we have used the local Ahlfors regularity, specifically, the (global) upper mass bound $\mu(B) \leq C_{0} \operatorname{rad}(B)^{Q}$. Next, we use local blanketedness of $\varphi$ to deduce that

$$
\operatorname{Mod}_{Q} \Gamma_{n} \leq C \sum_{j=1}^{N} \sum_{B \in \mathcal{C}_{j}} \varphi(B)^{Q} \leq C \sum_{j=1}^{N} V\left(\varphi^{Q}, \mathcal{C}_{j}\right) \leq C \cdot V\left(\varphi^{Q}, \mathcal{C}\right)
$$

where $C$ depends only on $t, Q, C_{0}, c_{0}$ and $M$. The proof is then completed by taking the infimum over all $\mathcal{V}$-full covers $\mathcal{C}$ of $X$. 
Proposition 8.9. Let $(X, \mu)$ be a locally compact, locally Ahlfors $Q$-regular metric measure space, $Q \geq 1$, with regularity constants $c_{0}$ and $C_{0}$. Let $c_{1}=c_{1}\left(Q, c_{0}, C_{0}\right)$ be a constant of local uniform perfectness for $X$. Finally, let $k \geq 1$.

Then there exists $K_{2} \geq 1$ depending only on $k, Q$ and $c_{0}$ so that for every $l>k$ and every l-admissible pair $(\mathcal{V}, \tilde{\mathcal{V}}) \subset \mathcal{B}_{k}(X)$, we have

$$
\bmod _{Q,(k, l, M)}^{\bullet} \Gamma \leq K_{2} \operatorname{Mod}_{Q} \Gamma
$$

for every $M \geq l / c_{1}$ and every curve family $\Gamma$.

Again our proof follows that of the corresponding result (Proposition 4.7) in [69. The arguments in the proof of Proposition 8.9 are in some sense "dual" to those in the proof of Proposition 8.2 Definition (8.11) "discretizes" a given admissible metric $\rho$ for the classical modulus, yielding a function $\varphi$ on $\mathcal{V} \cup \tilde{\mathcal{V}}$ which is admissible for the generalized modulus. In order to deal with the case when $X$ has infinite measure, we must introduce a slight modification in our definition of $\varphi$ in (8.11).

Since $(X, \mu)$ satisfies the global upper mass bound (1.2) by assumption, we may choose a positive Borel function $h \in L^{Q}(X, \mu)$ which is bounded away from zero on each bounded subset of $X$. For example, we may take $h(x)=H\left(\left|x-x_{0}\right|\right)$, where $x_{0} \in X$ is a fixed basepoint and $H:[0, \infty) \rightarrow(0, \infty)$ is any decreasing Borel function for which the sum

$$
\sum_{j=0}^{\infty} 2^{j Q} H\left(2^{j}\right)^{Q}
$$

converges.

Proof of Proposition 8.9. Let $\Gamma$ be a family of curves in $X$ and let $\rho$ be an admissible metric for $\operatorname{Mod}_{Q} \Gamma$. Without loss of generality, we may assume that $\rho$ is in $L^{Q}(X, \mu)$ and then, by Proposition 1.4(v), we may also assume that $\rho$ is lower semicontinuous.

In order to deal with the nonlocally rectifiable curves in $\Gamma$, we would like to be able to assume in addition that $\rho$ is bounded away from zero. In general, we cannot make this assumption unless $X$ has finite measure. We overcome this difficulty by introducing the above function $h$ into the following definition.

For $\tau>0$, define a function $\varphi=\varphi_{\tau}: \mathcal{V} \cup \tilde{\mathcal{V}} \rightarrow[0, \infty]$ by

$$
\varphi(A)=\inf _{x \in A}(\rho(x)+\tau h(x)) \cdot \operatorname{diam} A .
$$

We claim first that $\varphi$ is locally $M$-blanketed for $M \geq l / c_{1}$. Indeed, since $X$ is locally $c_{1}$-uniformly perfect, the collection of sets $A$ in $\mathcal{V}$ for which

$$
\operatorname{diam} \tilde{A} \leq\left(l / c_{1}\right) \operatorname{diam} A
$$

is a $\mathcal{V}$-full cover of $X$.

Next, we claim that $\varphi$ is an admissible set function for the generalized modulus of the curve family $\Gamma$. Consider first the case when $\gamma$ is not locally rectifiable. Then there exists a compact subinterval $I_{0} \subset I$ for which the associated subcurve is not rectifiable. The image $\left|\gamma_{0}\right|$ is contained in a bounded set $K \subset X$. Since $h_{K}:=\inf _{x \in K} h(x)>0$, Lemma 5.5 implies that

$$
\Phi \text {-length } k, \tilde{\mathcal{V}}(\gamma) \geq \Phi \text {-length }_{k, \tilde{\mathcal{V}}}\left(\gamma_{0}\right) \geq \tau h_{K} \operatorname{length}\left(\gamma_{0}\right)=\infty \geq 1 .
$$

On the other hand, suppose that $\gamma$ is locally rectifiable. In this case, we show that

$$
\int_{\gamma} \rho d s \leq \Phi \text {-length } k, \tilde{\mathcal{V}}(\gamma)
$$


Note that for a fixed curve $\gamma$,

$$
\int_{\gamma} \rho d s=\sup \int_{\gamma_{0}} \rho^{\prime} d s
$$

where the supremum is taken over all nonnegative continuous functions $\rho^{\prime}$ on $X$ satisfying $\rho^{\prime} \leq \rho$ and all closed subintervals $I_{0} \subset I$ with $\gamma_{0}$ rectifiable. (Here we make use of the fact that $\rho$ has been assumed to be lower semicontinuous.) Thus for the purposes of proving (8.12) we may assume that $\rho$ is continuous and that $\gamma$ is rectifiable with compact parametrizing interval.

The proof of (8.12) will be a modification of the proof of Lemma 5.5. Without loss of generality, assume that $\gamma: I=[0, L] \rightarrow X$ is parametrized by arc length. Since $|\gamma|$ is compact, we may choose $\epsilon>0$ so that the $\epsilon$-neighborhood $B(|\gamma|, \epsilon)$ is precompact in $X$. In this case, we have $h_{K}>0$, where $K=\bar{B}(|\gamma|, \epsilon)$.

Let $0<\beta<\tau h_{K}$. Since $\rho$ is uniformly continuous on $K$, we may assume (by making $\epsilon$ smaller if necessary) that $|\rho(x)-\rho(y)| \leq \beta$ whenever $x, y \in K$ satisfy $|x-y| \leq \epsilon$. Since $\rho$ is continuous, we may express the line integral of $\rho$ along $\gamma$ as a Riemann integral:

$$
\int_{\gamma} \rho d s=\lim _{\|\pi\| \rightarrow 0} \sum_{j=1}^{m} \rho\left(\tilde{\gamma}\left(t_{j}\right)\right) \cdot\left|\tilde{\gamma}\left(t_{j}\right)-\tilde{\gamma}\left(t_{j-1}\right)\right|,
$$

where the limit is taken over all partitions $\pi=\left\{0=t_{0}<t_{1}<\cdots<t_{m}=L\right\}$ of $I$ such that the mesh $\|\pi\|=\max \left\{t_{j}-t_{j-1}: j=1,2, \ldots m\right\}$ tends to zero. Choose a partition $\pi$ with mesh at most $\epsilon / 2$ for which

$$
\int_{\gamma} \rho d s-\beta \leq \sum_{j=1}^{m} \rho\left(\gamma\left(t_{j}\right)\right) \cdot\left|\gamma\left(t_{j}\right)-\gamma\left(t_{j-1}\right)\right| .
$$

By removing terms if necessary, we may assume that $\gamma\left(t_{j}\right) \neq \gamma\left(t_{j-1}\right)$ for each $j=1,2, \ldots, m$. Let $\delta_{0}$ denote the minimum of the quantities $\left|\gamma\left(t_{j}\right)-\gamma\left(t_{j-1}\right)\right|$, $j=1, \ldots, m$, and let $I_{j}$ denote the interval $\left[t_{j-1}, t_{j}\right]$. Let $0<\delta<\min \left\{\delta_{0}, \epsilon / 2\right\}$.

If $A \in \tilde{\mathcal{V}}^{\delta}$ meets $\gamma\left(I_{j}\right)$, then

$$
\rho\left(\gamma\left(t_{j}\right)\right) \cdot \operatorname{diam} A \leq \varphi(A) .
$$

Indeed, suppose that $\gamma(t) \in A \cap \gamma\left(I_{j}\right)$. For any $a \in A$, we have

$$
\left|a-\gamma\left(t_{j}\right)\right| \leq|a-\gamma(t)|+\left|\gamma(t)-\gamma\left(t_{j}\right)\right| \leq \delta+\left|t-t_{j}\right| \leq \epsilon / 2+\left|I_{j}\right| \leq \epsilon .
$$

Since $A \subset K$, we conclude that $\rho\left(\gamma\left(t_{j}\right)\right) \leq \rho(a)+\beta<\rho(a)+\tau h_{K}$. Taking the infimum over all such $a$ yields

$$
\rho\left(\gamma\left(t_{k}\right)\right) \cdot \operatorname{diam} A \leq \inf _{a \in A}(\rho(a)+\tau h(a)) \cdot \operatorname{diam} A=\varphi(A) .
$$

This proves (8.13). Returning to the main proof, let $\left\{\left(J_{\lambda}, A_{\lambda}\right)\right\}_{\lambda \in \Lambda}$ be an arbitrary parameterized cover of $\gamma$ by sets $A_{\lambda} \in \mathcal{B}_{k}(X)$ with outer radii $r_{2}\left(A_{\lambda}\right) \leq \delta$ and consider the quantity

$$
\sum_{j=1}^{m} \rho\left(\gamma\left(t_{j}\right)\right) \cdot\left|\gamma\left(t_{j}\right)-\gamma\left(t_{j-1}\right)\right|
$$

As in the proof of Lemma[5.5. by passing to a minimal finite subcover with index set $\Lambda^{\prime} \subset \Lambda$, we may ensure that each of the intervals $J_{\lambda}, \lambda \in \Lambda^{\prime}$, contains at most one 
of the $t_{j}$. Using the triangle inequality, we bound each expression $\left|\gamma\left(t_{j}\right)-\gamma\left(t_{j-1}\right)\right|$ by a sum of terms of the form diam $A_{\lambda}$. By (8.13) we have

$$
\sum_{j=1}^{m} \rho\left(\gamma\left(t_{j}\right)\right) \cdot\left|\gamma\left(t_{j}\right)-\gamma\left(t_{j-1}\right)\right| \leq \sum_{\lambda \in \Lambda^{\prime}} \varphi\left(A_{\lambda}\right)+(m+1) L \delta
$$

where $L=\sup \{\rho(x): x \in|\gamma|\}<\infty$. Here (as in (5.7)) the second term on the right-hand side is necessary to account for the sets covering each of the points $\gamma\left(t_{0}\right), \gamma\left(t_{1}\right), \ldots, \gamma\left(t_{m}\right)$. We conclude that

$$
\int_{\gamma} \rho d s-\beta \leq \Phi \text {-length }{ }_{k, \tilde{\mathcal{V}}}^{\delta}(\gamma)+(m+1) L \delta .
$$

Letting first $\delta$ and then $\beta$ tend to zero establishes $(8.12$. Thus $\varphi$ is an admissible function for the generalized modulus of $\Gamma$ and so

$$
\bmod _{Q,(k, l, M)}^{\bullet} \Gamma \leq \Phi_{Q, k, \mathcal{V}}^{\bullet}(X) .
$$

Finally, we claim that

$$
\Phi_{Q, k, \mathcal{V}}^{\bullet}(X) \leq K_{2} \int_{X}(\rho+\tau h)^{Q} d \mu
$$

for some constant $K_{2}$ depending only on $k, Q$ and $c_{0}$. Once we have shown this, the proof of the proposition is easily completed by letting $\tau$ tend to zero and taking the infimum over all admissible $\rho$.

To see why (8.14) holds, let $\mathcal{C}$ denote the collection of those sets $A$ in $\mathcal{B}_{k}(X)$ for which $\mu(A) \geq c_{0}^{\prime}(\operatorname{diam} A)^{Q}$, where $c_{0}^{\prime}=(2 k)^{-Q} c_{0}$. Local Ahlfors regularity implies that $\mathcal{C}$ is a $\mathcal{V}$-full cover of $X$. If $\left\{A_{i}\right\}$ is an arbitrary packing of $X$ by elements of $\mathcal{C}$, then

$$
\begin{aligned}
\sum_{i} \varphi\left(A_{i}\right)^{Q} & \leq\left(c_{0}^{\prime}\right)^{-1} \sum_{i}\left(\inf _{x \in A_{i}}(\rho(x)+\tau h(x))\right)^{Q} \mu\left(A_{i}\right) \\
& \leq\left(c_{0}^{\prime}\right)^{-1} \sum_{i} \int_{A_{i}}(\rho+\tau h)^{Q} d \mu \leq\left(c_{0}^{\prime}\right)^{-1} \int_{X}(\rho+\tau h)^{Q} d \mu .
\end{aligned}
$$

Thus $V\left(\varphi^{Q}, \mathcal{C}\right) \leq\left(c_{0}^{\prime}\right)^{-1} \int_{X}(\rho+\tau h)^{Q} d \mu$ and (8.14) follows with $K_{2}=\left(c_{0}^{\prime}\right)^{-1}$.

\section{EXPANSION OF THE GENERALIZED MOdULUS AND THE MAIN RESUlT}

Here we complete the proof of Theorem 6.4. Let $f: X \rightarrow Y$ be a homeomorphism satisfying (6.5) for some $t>1$. Choose any $l>t$ and $l^{\prime}=k^{\prime}>H$ and define a collection $\mathcal{V}=\mathcal{V}(f)$ of balls in $X$ as follows:

$B=\bar{B}(x, r)$ is in $\mathcal{V}$ provided the image $R^{\prime}=f(R)$ of the $l$-ring $R=$

$(B, t B)$ in $X$ centered at $x$ is an $l^{\prime}$-quasiring in $Y$ centered at $f(x)$.

By using (6.5), it is easy to see that $\mathcal{V}$ is a fine subcollection of $\mathcal{B}_{1}(X)$. Moreover, since $f$ is a homeomorphism, $f \mathcal{V}$ is a fine subcollection of $\mathcal{B}_{k^{\prime}}(Y)$. The pair $(\mathcal{V}, t \mathcal{V})$, resp. $(f \mathcal{V}, f(t \mathcal{V}))$ is $l$-, resp. $l^{\prime}$-admissible.

Proposition 9.1. Let $f: X \rightarrow Y$ be a homeomorphism of locally compact metric spaces satisfying

$$
\liminf _{r \rightarrow 0} \frac{L_{f}(x, t r)}{l_{f}(x, r)} \leq H
$$


for all $x \in X$ for some $t>1$ and $H<\infty$. Choose $l, \mathcal{V}$ and $l^{\prime}$ as above and let $M<\infty$. Then

$$
\bmod _{p, \sigma}^{\bullet} \Gamma \leq \bmod _{p, \sigma^{\prime}}^{\bullet} f \Gamma
$$

for any $p>0$ and any family of curves $\Gamma$ in $X$. Here in (9.2) the generalized modulus of $\Gamma$ is calculated with respect to the data $\sigma=(1, l, M)$ and the $l$-admissible pair $(\mathcal{V}, t \mathcal{V})$, while the generalized modulus of $f \Gamma$ is calculated with respect to the data $\sigma^{\prime}=\left(k^{\prime}, l^{\prime}, M\right)$ and the $l^{\prime}$-admissible pair $(f \mathcal{V}, f(t \mathcal{V}))$.

Compare [69, Theorem 3.24].

Proof. Let $\psi: f \mathcal{V} \cup f(t \mathcal{V}) \rightarrow[0, \infty]$ be a locally $M$-blanketed function satisfying

$$
\Psi \text {-length }{ }_{l^{\prime}, f(t \mathcal{V})}(f \gamma) \geq 1
$$

for each $\gamma \in \Gamma$. Define a function $\varphi: \mathcal{V} \cup t \mathcal{V} \rightarrow[0, \infty]$ by

$$
\varphi(A)=\psi(f A) .
$$

The function $\varphi$ is also locally $M$-blanketed. We claim that

(i) $\Phi$-length $\operatorname{tiv}_{1, t}(\gamma) \geq \Psi$-length ${ }_{l^{\prime}, f(t \mathcal{V})}(f \gamma)$ for each $\gamma \in \Gamma$;

(ii) $\Phi_{p, 1, \mathcal{V}}^{\bullet}(E) \leq \Psi_{p, l^{\prime}, f \mathcal{V}}^{\bullet}(f E)$ for each Borel set $E \subset X$.

These two facts clearly suffice to establish the desired result.

To prove (i), note that it suffices by (5.3) to assume that $\gamma$ has a compact parametrizing interval. Let $\delta^{\prime}>0$. Again, choose $\epsilon>0$ so that $B(|\gamma|, \epsilon)$ is precompact and set $K=\bar{B}(|\gamma|, \epsilon)$. Since $f$ is uniformly continuous on $K$, we may choose $\delta>0$ so that for any set $A \in t \mathcal{V}$ with outer radius at most $\delta$ which meets $|\gamma|$ the following two conditions hold: $A \subset K$ and $f A \in f(t \mathcal{V})$ has outer radius at most $\delta^{\prime}$.

If $\mathcal{C}=\left\{\left(I_{\lambda}, A_{\lambda}\right)\right\}$ is a parametrized cover of $\gamma$ by sets $A_{\lambda} \in t \mathcal{V}$ with outer radius at most $\delta$, then $f \mathcal{C}$ is a parametrized cover of $f \gamma$ and $A_{\lambda}^{\prime}=f A_{\lambda}$ has outer radius at most $\delta^{\prime}$, which implies that

$$
\sum_{\lambda} \varphi\left(A_{\lambda}\right)=\sum_{\lambda} \psi\left(A_{\lambda}^{\prime}\right) \geq \Psi \text {-length } \text { l' }_{l^{\prime}, f(t \mathcal{V})}^{\delta^{\prime}}(f \gamma) .
$$

Taking the infimum over all such covers yields

$$
\Psi \text {-length }{ }_{l^{\prime}, f(t \mathcal{V})}^{\delta^{\prime}}(f \gamma) \leq \Phi \text {-length }{ }_{1, t \mathcal{V}}^{\delta}(\gamma) \leq \Phi \text {-length }{ }_{1, t \mathcal{V}}(\gamma)
$$

and the result follows by letting $\delta^{\prime} \rightarrow 0$.

The proof of (ii) is even easier. If $\mathcal{C}^{\prime}$ is a $f \mathcal{V}$-full cover of $f E$, then $\mathcal{C}$ is a $\mathcal{V}$-full cover of $E$ (since $f$ is a homeomorphism) and so

$$
\Phi_{p, 1, \mathcal{V}}^{\bullet}(E) \leq V\left(\varphi^{p}, \mathcal{C}\right) \leq V\left(\psi^{p}, \mathcal{C}^{\prime}\right)
$$

The proof is completed by taking the infimum over all such covers $\mathcal{C}^{\prime}$.

We now combine all of these results to prove Theorem 6.4. Fix any $l>t$ and $l^{\prime}=k^{\prime}>H$. Set $M=l / c_{1}^{\prime} \geq 1$, where $c_{1}^{\prime}$ is a constant of local uniform perfectness for $Y$. By Propositions $8.2,8.9$ and 9.1 ,

$$
\operatorname{Mod}_{Q} \Gamma \leq K_{1} \bmod _{Q,(1, l, M)}^{\bullet} \Gamma \leq K_{1} \bmod _{Q,\left(k^{\prime}, l^{\prime}, M\right)}^{\bullet} f \Gamma \leq K_{1} K_{2} \operatorname{Mod}_{Q} f \Gamma
$$

which establishes the desired result with $K=K_{1} K_{2}$.

Corollary 6.7 follows by combining Theorem 6.4 Lemma 9.3] and the elementary observation that the inverse of a locally $\eta$-quasisymmetric map $f$ is locally $\eta_{L^{-}}$ quasisymmetric, where $\eta_{L}(t)=\eta^{-1}\left(t^{-1}\right)^{-1}$. 
Lemma 9.3. Let $f: X \rightarrow Y$ be a locally $\eta$-quasisymmetric map between metric spaces and assume that $Y$ is locally $C_{1}-L L C$. Let $t \geq 1$. Then there exists a constant $H$ depending on $C_{1}, t$ and $\eta$ so that

$$
\limsup _{r \rightarrow 0} \frac{L_{f}(x, t r)}{l_{f}(x, r)} \leq H
$$

for all $x \in X$.

The proof of this lemma is completely standard and will be left as an exercise to the reader with the following hint: the linear local connectivity of the target space is necessary to derive (9.4) since the map is only assumed to be locally quasisymmetric. See the footnote to 6.2). (If $f$ is assumed to be globally quasisymmetric, then Lemma 9.3 holds for arbitrary metric spaces.)

Note also that we make no assumption a priori in Theorem 6.4 or Corollary 6.7 that the spaces $X$ and $Y$ support any locally rectifiable curves (of course, in the case when neither space supports any locally rectifiable curves, the geometric quasiconformality is a trivial condition). We deduce that the existence of a sufficient large family of rectifiable curves in $X$ necessarily implies the existence of a corresponding family in $Y$. These remarks are made more precise in section 12

\section{The LOEWNER CONDITION AND GEOMETRIC QUASICONFORMALITY}

Definition 10.1. Let $X$ be a metric space and let $E, F, G$ be Borel sets in $X$. We denote by $(E, F ; G)$ the collection of all curves $\gamma$ joining $E$ to $F$ in $G$, that is, $\gamma: I \rightarrow X$ (where $I$ is an interval with endpoints $a, b$ in $\mathbb{R}$ ) satisfies $\gamma(t) \in G$ for $a<t<b, \overline{|\gamma|} \cap E \neq \emptyset$, and $\overline{|\gamma|} \cap F \neq \emptyset$. When $G=X$ we abbreviate $(E, F ; G)=(E, F)$.

The following concept, first introduced by Heinonen and Koskela in [38], has been recognized as a crucial property of metric spaces sufficient to guarantee a Euclidean-type theory of quasiconformal maps.

Definition 10.2. Let $(X, \mu)$ be a rectifiably connected metric measure space and let $Q \geq 1$. We say that $X$ is a Loewner space (with exponent $Q$ ) if there exists a nonincreasing function $\phi:(0, \infty) \rightarrow(0, \infty)$ so that

$$
\operatorname{Mod}_{Q}(E, F) \geq \phi(t)
$$

whenever $E$ and $F$ are disjoint, nondegenerate continua in $X$ for which

$$
\operatorname{dist}(E, F) \leq t \min \{\operatorname{diam} E, \operatorname{diam} F\} .
$$

We call $\phi$ a Loewner function for $X$.

Remark 10.3. For any $n \geq 1$, the Euclidean space $\mathbb{R}^{n}$ is a Loewner space (with exponent $Q=n)$. This is trivial in the case $n=1$ since $\operatorname{Mod}_{1}(E, F ; \mathbb{R}) \geq 1$ for any two disjoint compact sets $E$ and $F$ in $\mathbb{R}$. When $n=2$ the estimate is classical. Loewner [47] established the case $n \geq 3$ in qualitative form, while Gehring [28] developed important symmetrization techniques to prove the quantitative version.

Further examples of Loewner spaces will be discussed in section [1] additional examples are given in [38, Section 6].

Every metric measure space satisfying the Loewner condition for some $Q>1$ necessarily also satisfies the lower mass bound (1.3) for some constant $c_{0}$. (Thus $Q \geq \operatorname{dim}_{H} X$.) If the space in addition satisfies the upper mass bound (1.2) for 
some $C_{0}<\infty$, then it is $C_{1}$-LLC and $C_{2}$-quasiconvex: every pair of points $x$ and $y$ in $X$ can be joined by a curve whose length is no more than $C_{2}|x-y|$. These statements are quantitative in the usual sense (the constants $c_{0}, C_{1}$ and $C_{2}$ depend only on the Loewner function $\phi$ and on $Q$ ). For proofs of these results, see [38 §3].

The importance of the Loewner condition is evident in the following proposition, which establishes local quasisymmetry for mappings satisfying the conclusion of Theorem 6.4 The argument is well-known in quasiconformal function theory (see e.g. [38 Theorem 4.7]). For simplicity, we state the result only in the case of open subsets of $Q$-regular spaces.

Proposition 10.4. Let $(X, \mu)$ be a $Q$-regular Loewner space and let $(Y, \nu)$ be a $Q$ regular $L L C$ space, $Q>1$. Let $f: U \rightarrow U^{\prime}$ be a homeomorphism between open sets $U \subset X$ and $U^{\prime} \subset Y$ which satisfies $\operatorname{Mod}_{Q} \Gamma \leq K \operatorname{Mod}_{Q} f \Gamma$ for all curve families $\Gamma$ in $U$. Then $f$ is locally quasisymmetric.

We begin with a lemma which states that open subsets of $Q$-regular Loewner spaces satisfy a weak local version of the Loewner condition.

Lemma 10.5. Let $U$ be an open subset of a $Q$-regular Loewner space $X, Q>1$. Let $t_{0}>0$. Then there exists a constant $a=a\left(t_{0}, \phi, Q\right)>0$ so to each point $x_{0} \in U$ there corresponds a ball $B_{0} \subset U$ with the following property: if $E$ and $F$ are disjoint, nondegenerate continua in the dilated ball $a B_{0}$ which satisfy

$$
\operatorname{dist}(E, F) \leq t_{0} \min \{\operatorname{diam} E, \operatorname{diam} F\},
$$

then $\operatorname{Mod}_{Q}\left(E, F ; B_{0}\right) \geq \frac{1}{2} \phi\left(t_{0}\right)>0$.

In the proofs of Proposition 10.4 and Lemma 10.5, we make use of the following estimate for the $Q$-modulus valid in any metric measure space $X$ satisfying the upper mass bound with exponent $Q$ : there exists a constant $C$ (depending only on $Q$ and the upper mass bound constant $C_{0}$ ) so that

$$
\operatorname{Mod}_{Q}\left(\bar{B}\left(x_{0}, r_{1}\right), X \backslash B\left(x_{0}, r_{2}\right)\right) \leq C\left(\log r_{2} / r_{1}\right)^{1-Q}
$$

whenever $x_{0} \in X$ and $0<2 r_{1}<r_{2}<\infty$ (see, for example, 38, Lemma 3.14]).

Proof of Lemma 10.5. Let $\phi$ be the Loewner function for $X$. Choose $a$ so small that

$$
C(\log 1 / a)^{1-Q} \leq \frac{1}{2} \phi\left(t_{0}\right)
$$

where $C$ is the constant in (10.6). Let $B_{0}$ be any ball centered at $x_{0}$ and contained in $U$. If $E$ and $F$ are as in the hypotheses, then

$$
\phi\left(t_{0}\right) \leq \operatorname{Mod}_{Q}(E, F) \leq \operatorname{Mod}_{Q}\left(E, F ; B_{0}\right)+\operatorname{Mod}_{Q}\left(E \cup F, X \backslash B_{0}\right) .
$$

Since $\left(E \cup F, X \backslash B_{0}\right)$ is minorized by the curve family $\left(a B_{0}, X \backslash B_{0}\right)$, we see by using (10.6) that

$$
\phi\left(t_{0}\right) \leq \operatorname{Mod}_{Q}\left(E, F ; B_{0}\right)+C(\log 1 / a)^{1-Q}
$$

and the result follows.

Proof of Proposition 10.4. Assume that $Y$ is $C_{1}$-LLC. Let $x_{0} \in U$. Choose $a>0$ and a ball $B_{0}$ centered at $x_{0}$ and contained in $U$ so that the conclusion of Lemma 10.5 holds with $t_{0}=2$. Pick $r_{0}>0$ so that

$$
\bar{B}\left(f x_{0},\left(2 C_{1}+1\right) L_{f}\left(x_{0}, 2 r_{0}\right)\right) \subset f\left(a B_{0}\right) \text {. }
$$


Such an $r_{0}$ can always be found since $f: U \rightarrow U^{\prime}$ is a homeomorphism. Furthermore, since we may choose the ball $B_{0}$ to be as small as we please (see the proof of Lemma 10.5), we may assume in addition that

$$
\bar{B}\left(f x_{0}, L_{f}\left(x_{0}, 2 r_{0}\right)\right) \neq U^{\prime} .
$$

Note that $B\left(x_{0}, 2 r_{0}\right) \subset a B_{0}$ since $f\left(B\left(x_{0}, 2 r_{0}\right)\right) \subset B\left(f x_{0}, L_{f}\left(x_{0}, 2 r_{0}\right)\right) \subset f\left(a B_{0}\right)$ by (10.7).

Let $V^{\prime}$ be the component of $f B\left(x_{0}, r_{0}\right)$ containing $f x_{0}$ and let $V=f^{-1} V^{\prime}$. Note that $V^{\prime}$ is open since $Y$ is LLC. We will show that $\left.f\right|_{V}$ is weakly quasisymmetric, that is, there exists $H<\infty$ so that

$$
|x-y| \leq|x-z| \Rightarrow|f x-f y| \leq H|f x-f z|, \quad x, y, z \in V .
$$

Since $V \subset X$ and $f V \subset Y$ are doubling spaces and $V$ is connected, it follows (see e.g. [73 Theorem 2.9]) that $\left.f\right|_{V}$ is quasisymmetric.

Let $x, y, z \in V$ with $|x-y| \leq|x-z|$. Set $s=|f x-f z|$ and $S=|f x-f y|$. Note that $f z \in \bar{B}(f x, s)$ and $f y \in U^{\prime} \backslash B(f x, S)$. Choose a point $w \in U \backslash \bar{B}\left(x_{0}, 2 r_{0}\right)$ with $f w \in U^{\prime} \backslash \bar{B}\left(f x, \frac{1}{2} S\right)$. This can be done since

$$
S=|f x-f y| \leq\left|f x-f x_{0}\right|+\left|f x_{0}-f y\right| \leq 2 L_{f}\left(x_{0}, 2 r_{0}\right)
$$

and (10.8) implies that there exists $w^{\prime}=f w \in U^{\prime}$ with $\left|f x_{0}-f w\right|>2 L_{f}\left(x_{0}, 2 r_{0}\right)$ which in turn implies that $|f x-f w|>L_{f}\left(x_{0}, 2 r_{0}\right) \geq \frac{1}{2} S$.

Join $f x$ to $f z$ with a continuum $E^{\prime} \subset \bar{B}\left(f x, C_{1} s\right)$ and join $f y$ to $f w$ with a continuum $F^{\prime} \subset Y \backslash B\left(f x, \frac{1}{2 C_{1}} S\right)$. Since $s \leq 2 L_{f}\left(x_{0}, r_{0}\right)$ we have

$$
E^{\prime} \subset \bar{B}\left(f x_{0}, C_{1} s+L_{f}\left(x_{0}, r_{0}\right)\right) \subset \bar{B}\left(f x_{0},\left(2 C_{1}+1\right) L_{f}\left(x_{0}, r_{0}\right)\right) \subset f\left(a B_{0}\right) .
$$

We cannot guarantee, however, that $F^{\prime}$ is contained in $f\left(a B_{0}\right)$.

Let $E=f^{-1} E^{\prime}$ and let $F=f^{-1} F^{\prime \prime}$, where $F^{\prime \prime}$ is the component of $F^{\prime}$ lying in $f\left(a B_{0}\right)$ which contains $f y$. Then $E$ contains $x$ and $z$ while $F$ contains $y$. We consider two cases:

(i) $F^{\prime} \subset f\left(a B_{0}\right)$. In this case $F^{\prime \prime}=F$ and so $F$ contains both $y$ and $w$. Hence $\operatorname{diam} F \geq|y-w| \geq\left|w-x_{0}\right|-\left|y-x_{0}\right| \geq r_{0}$

(ii) $F^{\prime}$ meets $Y \backslash f\left(a B_{0}\right)$. In this case $\bar{F}$ meets $X \backslash a B_{0}$; say $q \in \bar{F} \cap\left(X \backslash a B_{0}\right)$. Thus $\operatorname{diam} F \geq|q-y| \geq\left|q-x_{0}\right|-\left|y-x_{0}\right| \geq r_{0}$.

In either case we conclude that $\operatorname{diam} F \geq r_{0}$ and so

$$
\frac{\operatorname{dist}(E, F)}{\min \{\operatorname{diam} E, \operatorname{diam} F\}} \leq \frac{|x-y|}{\min \left\{|x-z|, r_{0}\right\}} \leq 2 .
$$

Since $E$ and $F$ are contained in $a B_{0}, \operatorname{Mod}_{Q}\left(E, F ; B_{0}\right) \geq \frac{1}{2} \phi\left(t_{0}\right)$ and so

$$
0<(2 K)^{-1} \phi\left(t_{0}\right) \leq \operatorname{Mod}_{Q} f\left(E, F ; B_{0}\right)=\operatorname{Mod}_{Q}\left(E^{\prime}, F^{\prime \prime} ; f\left(B_{0}\right)\right) .
$$

But the curve family $\left(E^{\prime}, F^{\prime \prime} ; f\left(B_{0}\right)\right)$ is minorized by the family $\left(\bar{B}\left(f x, C_{1} s\right)\right.$, $\left.Y \backslash B\left(f x, S / 2 C_{1}\right)\right)$. If $S>4 C_{1}^{2} s$, we deduce (by applying (10.6) in the image $Y)$ that

$$
0<(2 K)^{-1} \phi\left(t_{0}\right) \leq C\left(\log \left(S /\left(2 C_{1}^{2} s\right)\right)\right)^{1-Q}
$$

and we conclude that $S / s$ is bounded from above by a constant depending only on $C_{1}, K, \phi, Q$, and the regularity constants of $Y$. 
Theorem 10.9. Let $X$ and $Y$ be locally compact, Ahlfors $Q$-regular spaces, $Q>1$. Assume that $X$ is Loewner and $Y$ is LLC and that $f: U \rightarrow U^{\prime}$ is a homeomorphism between open sets $U \subset X$ and $U^{\prime} \subset Y$. Then the following conditions on $f$ are equivalent:

(i) there exists $t>1$ and $H<\infty$ so that

$$
\liminf _{r \rightarrow 0} \frac{L_{f}(x, t r)}{l_{f}(x, r)} \leq H
$$

for all $x$ in $X$;

(ii) for each $t>1$, there exists $H(t)<\infty$ so that

$$
\limsup _{r \rightarrow 0} \frac{L_{f}(x, t r)}{l_{f}(x, r)} \leq H(t)
$$

for all $x$ in $X$;

(iii) there exists $K<\infty$ so that $f$ is geometrically $K$-quasiconformal;

(iv) there exists $\eta:[0, \infty) \rightarrow[0, \infty)$ so that $f$ is locally $\eta$-quasisymmetric.

Theorem 10.9 follows by combining Theorem 6.4. Lemma 9.3 and Proposition 10.4. Indeed, Theorem 6.4 shows that (i) implies "half" of (iii), namely, the onesided inequality

$$
\operatorname{Mod}_{Q} \Gamma \leq K \operatorname{Mod}_{Q} f \Gamma
$$

for curve families $\Gamma$ in $X$, while Proposition 10.4 shows that 10.10 implies (iv). On the other hand, (iv) implies (ii) by Lemma 9.3 and (ii) implies (i) trivially. Since (iv) holds for a function $f$ if and only if it holds for the inverse function $f^{-1}$, we see that the corresponding conditions also hold for $f^{-1}$. In particular, (10.10) also holds for $f^{-1}$ and so the two-sided inequality

$$
\frac{1}{K} \operatorname{Mod} \Gamma \leq \operatorname{Mod}_{Q} f \Gamma \leq K \operatorname{Mod}_{Q} \Gamma
$$

holds for curve families $\Gamma$ in $X$, i.e., (iii) is valid.

Remark 10.12. In the Euclidean case, Theorem 10.9 is essentially the result of Gehring in 29] (modulo only the $t>1$ versus $t=1$ issue, which has already been discussed). For mappings between domains in the Heisenberg group, the equivalence given in Theorem 10.9 is due to Korányi and Reimann [43. However, as has been emphasized before, the proof of Theorem 10.9 given here is new even in these settings. Previous proofs of the equivalence of geometric quasiconformality with various metric definitions have made use of the absolute continuity of quasiconformal maps. Our proof makes no use of these properties, in fact, they can be derived as consequences of Theorem 10.9 (see section 14).

\section{Examples of Ahlfors Regular Loewner spaces}

We briefly describe several non-Euclidean examples of Ahlfors regular Loewner spaces. All of these examples are "fractal" in the sense of Mandelbrot; the Hausdorff dimension strictly exceeds the topological dimension. None of the examples in this section are new to this paper; we include them here in order to provide concrete situations in which our results apply. For other examples, see [38, Section $6]$ and [33]. 
Example 11.1 (Carnot groups). As mentioned in the introduction, it was in the context of Carnot groups that non-Euclidean quasiconformal maps were first studied. Recall that a Carnot (or stratified) group $G$ is a connected and simply connected nilpotent Lie group with graded Lie algebra $\mathfrak{g}=V_{1} \oplus \cdots \oplus V_{r}$ satisfying $\left[V_{1}, V_{i}\right]=V_{i+1}$ for $i=1,2, \ldots, r-1$. We identify the elements of $\mathfrak{g}$ with left invariant vector fields on $G$. Each Carnot group $G$ is metrized via the CarnotCarathéodory metric:

$$
d_{C}(x, y):=\inf \int_{a}^{b}\left\langle\gamma^{\prime}(t), \gamma^{\prime}(t)\right\rangle_{\gamma(t)}^{1 / 2} d t, \quad x, y \in G
$$

where the infimum is taken over all curves $\gamma: I \rightarrow G$ with $\gamma(a)=x$ and $\gamma(b)=y$ for which $\gamma^{\prime}(t) \in V_{1}$ for all $t$ (see, e.g., [20] or [53]). The resulting metric space is a complete length space with topological dimension $N=\sum_{i=1}^{r} \operatorname{dim} V_{i}$. The Haar measure on $G$ (which arises as the lift of Lebesgue measure on $\mathfrak{g}$ under the exponential map) is Ahlfors $Q$-regular with respect to the Carnot-Carathéodory metric, where $Q=\sum_{i=1}^{r} i \cdot \operatorname{dim} V_{i}$ is the so-called homogeneous dimension of $G$. Note that $Q>N$ whenever $G$ is non-abelian, i.e., $r>1$. The simplest non-abelian Carnot groups are the so-called Heisenberg groups which arise in the Iwasawa decomposition of the isometry group of $n$-dimensional complex hyperbolic space.

Each Carnot group (equipped with the Carnot-Carathéodory metric $d_{C}$ and the Hausdorff measure $\mathcal{H}_{Q}$ ) satisfies the Loewner condition. In the case of the Heisenberg groups this fact is due to Reimann [55; the general case is due to Heinonen [35].

An interesting question to ask is whether or not nontrivial (i.e., nonconformal) quasiconformal mappings exist in general Carnot groups. Korányi and Reimann 43] have constructed nontrivial mappings on the Heisenberg groups (see also [2]; for a different approach, see [17], 63]). However, by a result of Pansu [52], in the Carnot groups which arise in the Iwasawa decompositions of the isometry groups of $n$-dimensional quaternionic hyperbolic spaces and the Cayley hyperbolic plane, every quasiconformal map is in fact conformal. That is, if a map $f$ on these spaces satisfies (0.3) for some $K<\infty$, then it satisfies (0.3) for $K=1$. Whether or not nontrivial quasiconformal maps exist in other Carnot groups appears to be an open question (but cf. [54], [56]).

For further information on quasiconformal mappings on Carnot groups, see [48, [36], [34], [15], [16], [5] and [4], in addition to the references already mentioned.

Example 11.2 (boundaries at infinity of two-dimensional hyperbolic buildings). Non-abelian Carnot groups are examples of Ahlfors regular Loewner spaces with Hausdorff dimension strictly exceeding the topological dimension. However, every Carnot group has integral Hausdorff dimension and for some time it was not known whether examples existed with nonintegral Hausdorff dimension [40, Questions 19 and 20]. Bourdon and Pajot [10] answered this question by establishing the Loewner condition for a class of metric spaces arising as the boundaries at infinity of certain two-dimensional hyperbolic buildings. We briefly recall their construction.

Let $p \geq 4$ and $q \geq 2$ be integers. Denote by $I_{p, q}$ the unique simply connected cellular 2-complex with the following properties: (i) each of its 2-cells is a regular hyperbolic $p$-gon (a regular Euclidean square if $p=4$ ) with interior angles $\pi / 2$, (ii) 
any two 2-cells share at most one edge or one vertex, and (iii) the link 10 of each vertex is the complete bipartite graph $K_{q, q}$ on $2 q$ vertices (see Figureप).
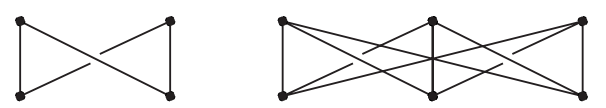

Figure 1. The complete bipartite graphs $K_{2,2}$ and $K_{3,3}$. [From M. Bourdon, Immeubles hyperboliques, dimension conforme et rigidité de Mostow, Geometric and Functional Analysis, vol. 7 (1997), pp. 245-268. Used with permission.]

In Figure 2 we reproduce [9, Figure 2], showing the link of a vertex $V$ in the complex $I_{p, 3}$. (For simplicity, the hyperbolic $p$-gons attached to $V$ are represented in Figure 2 by triangles.)

For the existence and uniqueness of the space $I_{p, q}$, see [9]. When $p \geq 5$ and $q \geq 3$, the space $I_{p, q}$ is a Gromov hyperbolic space [21] and thus admits a Gromov boundary at infinity $\partial I_{p, q}$. By [18, Théorème 4.17] (see also [6, p. 66]) this boundary is homeomorphic to the Menger sponge (see Figure 3). In particular, $\partial I_{p, q}$ has (topological) dimension one.

Remark 11.3. When $q=2, I_{p, 2}$ reduces to the hyperbolic plane together with its standard tiling by regular hyperbolic $p$-gons. In this case, $\partial I_{p, 2}$ can be identified with the unit circle $S^{1}$. When $p=4, I_{4, q}$ is a Euclidean building whose chambers are copies of a (Euclidean) square; in this case $I_{4, q}$ corresponds to the product of two regular trees of valence $q$.

For each $p \geq 5$ and $q \geq 3, \partial I_{p, q}$ is equipped with a natural metric $d_{p, q}$ for which $\left(\partial I_{p, q}, d_{p, q}\right)$ is a geodesic space which is Ahlfors regular of dimension

$$
Q_{p, q}=1+\frac{\log (q-1)}{\operatorname{arccosh}\left(\frac{p-2}{2}\right)}>1
$$

(see [9, Lemme 3.1.4]). Bourdon and Pajot [10] have shown that for $p \geq 5$ and $q \geq 3,\left(\partial I_{p, q}, d_{p, q}, \mathcal{H}_{Q_{p, q}}\right)$ satisfies the Loewner condition.

Remark 11.5. For each $p \geq 5$ and $q \geq 3, Q_{p, q}$ is irrational, moreover, the set of allowable dimensions $Q_{p, q}$ in (11.4) is dense in the interval [1, $)$ ). The mapping $(p, q) \mapsto Q_{p, q}$ from $\{(p, q) \in \mathbb{N} \times \mathbb{N}: p \geq 5, q \geq 3\}$ to $(1, \infty)$ is not injective, e.g.,

$$
Q_{5,3}=1+\frac{\log 2}{\operatorname{arc} \cosh (3 / 2)}=1+\frac{\log 4}{\operatorname{arc~cosh}(7 / 2)}=Q_{9,5} \approx 1.72021 \ldots
$$

For $N \geq 1$, let $T_{N}$ denote the $N$ th Chebyshev polynomial, i.e., the unique polynomial of degree $N$ satisfying

$$
\cosh (N t)=T_{N}(\cosh t), \quad t>0 .
$$

\footnotetext{
${ }^{10}$ Recall that the $\operatorname{link}$ of a vertex $V$ in a 2-complex $C$ is the graph $G(V)$ whose vertices are the edges adjacent to $V$ and whose edges are the faces adjacent to $V$, where an edge in $G(V)$ joins two vertices in $G(V)$ if and only if the corresponding face in the complex $C$ contains the corresponding edges in its boundary.
} 


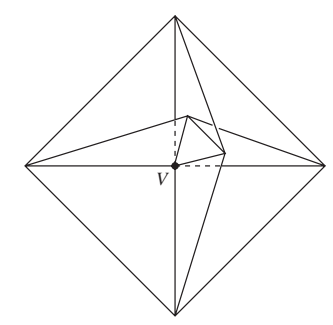

Figure 2. Combinatorial structure of the link of a vertex $V$ in the space $I_{p, 3}$. [From M. Bourdon, Immeubles hyperboliques, dimension conforme et rigidité de Mostow, Geometric and Functional Analysis, vol. 7 (1997), pp. 245-268. Used with permission.]

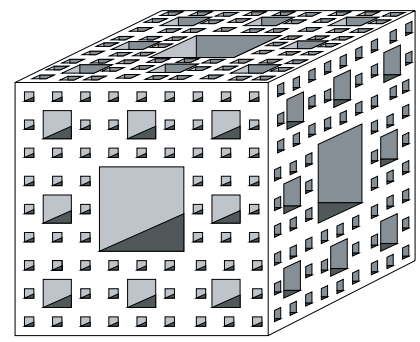

Figure 3. The Menger sponge

The recurrence relation $T_{N+1}(x)=2 x T_{N}(x)-T_{N-1}(x)$ for the Chebyshev polynomials implies that the quantity $2 T_{N}(n / 2)$ is an integer for each $n, N \in \mathbb{N}$. It follows that the values $p^{\prime}$ and $q^{\prime}$ defined by the expressions

$$
p^{\prime} / 2-1=T_{N}(p / 2-1), \quad q^{\prime}-1=(q-1)^{N},
$$

are integers for any choice of integers $p \geq 5, q \geq 3$, and $N$. In this case $Q_{p, q}=Q_{p^{\prime}, q^{\prime}}$.

Conjecture 11.6. $Q_{p, q}=Q_{p^{\prime}, q^{\prime}}$ if and only if there exist $M, N \in \mathbb{N}$ so that

$$
T_{M}\left(\frac{p^{\prime}-2}{2}\right)=T_{N}\left(\frac{p-2}{2}\right)
$$

and

$$
\left(q^{\prime}-1\right)^{M}=(q-1)^{N} .
$$

The spaces $\partial I_{p, q}$ and $\partial I_{p^{\prime}, q^{\prime}}$ are not quasisymmetrically equivalent whenever $Q_{p, q} \neq Q_{p^{\prime}, q^{\prime}}$. This follows from the fact that $\partial I_{p, q}$ satisfies the Loewner condition and hence has nontrivial $Q_{p, q}$-modulus, see Remark 15.6. Consequently, the hyperbolic buildings $I_{p, q}$ and $I_{p^{\prime}, q^{\prime}}$ are not quasi-isometric. This result is due to Bourdon [9, Théorème 1.1]. However, when $Q_{p, q}=Q_{p^{\prime}, q^{\prime}}$ we are at present unable to say whether or not the corresponding spaces are quasisymmetrically equivalent. Bourdon [9, Remarque, p. 247] conjectures that these spaces are always pairwise quasisymmetrically inequivalent.

Example 11.7 (Laakso's spaces). In [46], Laakso gives an explicit construction of an Ahlfors $Q$-regular Loewner space for each $Q>1$. His examples arise as quotients of the product of the unit interval with a Cantor set under a finite-to-one map. 
Let $F$ denote a space consisting of two elements, to which we assign the discrete topology. The abstract Cantor set $K=F^{\infty}$ consists of all countably infinite sequences $u=\left(u_{1}, u_{2}, \ldots\right)$, where $u_{i} \in F$ for each $i$. Endowed with the Tychonoff (product) topology, $K$ is a compact Hausdorff space of (topological) dimension zero.

Fix $\alpha \in(0,1)$ and let

$$
d_{\alpha}(u, v):=\alpha^{N(u, v)-1}, \quad u, v \in K,
$$

where $N(u, v)$ is the maximum value $n$ for which $u_{i}=v_{i}$ for $i=1,2, \ldots, n 11$ Then $d_{\alpha}$ is a metric on $K$, compatible with the above topology. For any $\alpha \in(0,1)$, the abstract Cantor set $\left(K, d_{\alpha}\right)$ is bi-Lipschitz equivalent to a Cantor set constructed by the standard method in some Euclidean space, e.g., $\left(K, d_{1 / 3}\right)$ is bi-Lipschitz equivalent to the usual one-third Cantor set in the real line.

Finally, let $\mu$ be the measure on $K$ given by the countable product of copies of the uniform probability measure which assigns weight $1 / 2$ to each of the elements of $F$. Then $\mu$ is a Borel measure on $K$ which is Ahlfors regular of dimension $\log 2 / \log (1 / \alpha)$.

Fix now a real number $Q>1$ and choose $\alpha \in(0,1)$ so that

$$
Q=1+\frac{\log 2}{\log (1 / \alpha)} \text {. }
$$

Let $I=[0,1]$ and consider the product space $I \times K$, endowed with the product metric $d_{\text {Eucl }} \times d_{\alpha}$ and the product measure $\mathcal{L}_{1} \times \mu$, where $\mathcal{L}_{1}$ denotes Lebesgue measure on the interval $I$. Then $I \times K$ is Ahlfors regular of dimension $Q$. Laakso's space is a quotient of $I \times K$ under a two-to-one mapping $s$ on $I \times K$.

Choose $p \in \mathbb{N}$ so that $1 /(p+1)<\alpha \leq 1 / p$. Next, choose a sequence of integers $l_{i} \in\{p, p+1\}, i=1,2, \ldots$, so that

$$
\left(\frac{p+1}{p}\right)^{-1} \alpha^{m} \leq L_{m}^{-1} \leq \frac{p+1}{p} \alpha^{m},
$$

where for each $m \in \mathbb{N}$, we set $L_{m}=\prod_{i=1}^{m} l_{i}$ (with the convention $L_{0}=1$ ) 12

For each $m=0,1,2, \ldots$, let $\mathcal{I}_{m}$ denote the collection of points of the form $k \cdot L_{m}^{-1}$, $k=0,1, \ldots, L_{m}$, and for each $m=1,2, \ldots$, let $\mathcal{J}_{m}=\mathcal{I}_{m} \backslash \mathcal{I}_{m-1}$. Then $\mathcal{J}_{m}$ is $L_{m}^{-1}$-dense in $I$ (any point in $I$ is within $L_{m}^{-1}$ from some point in $\mathcal{J}_{m}$ ) and $\mathcal{I}_{m}$ is $L_{m}^{-1}$-discrete $\left(\left|t-t^{\prime}\right| \geq L_{m}^{-1}\right.$ whenever $\left.t, t^{\prime} \in \mathcal{I}_{m}, t \neq t^{\prime}\right)$.

We identify two points $p=(a, u)$ and $q=(b, v)$ in $I \times K$, writing $p \sim q$, if there exists $m \in \mathbb{N}$ so that $a=b \in \mathcal{J}_{m}$ and $u_{i}=v_{i}$ for all $i \neq m$. Note that no equivalence class consists of more than two elements. Also, if $p \sim q$ and $m$ are as above, then $d_{\alpha}(u, v) \leq \alpha^{m-1}$. Figure 4 shows some of the identifications in the case when $K$ is the standard one-third Cantor set in the real line $(\alpha=1 / 3)$ and $l_{1}=l_{2}=\cdots=3$. (The two endpoints of each dotted line are identified in the quotient space.)

Let $X=I \times K / \sim$ be the quotient space and let $s: I \times K \rightarrow X$ be the quotient map. Topologized with the quotient topology, $X$ is a compact Hausdorff space. For $x, y \in X$, set

$$
d(x, y)=\inf \sum_{\nu} \operatorname{length}\left(\gamma_{\nu}\right)
$$

\footnotetext{
${ }^{11}$ We allow here the possibility that $n=0$ (if $u_{1} \neq v_{1}$ ) or $n=\infty$ (if $u_{i}=v_{i}$ for all $i$ ).

${ }^{12}$ The coefficient $(p+1) / p$ in (11.8) is not crucial (any fixed constant would work), however, $(p+1) / p$ is the smallest value for which such a sequence $l_{1}, l_{2}, \ldots$ must necessarily exist.
} 


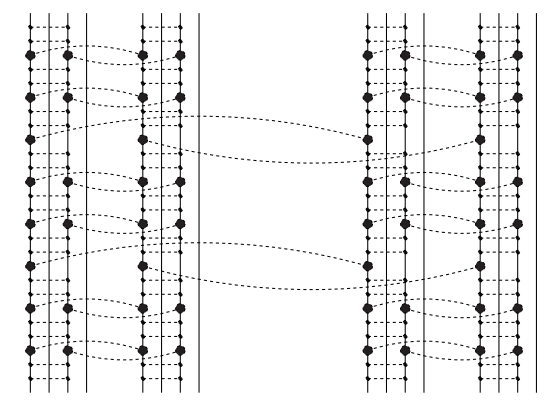

FiguRE 4. Identifications in the Laakso space

where the infimum is taken over all geodesic chains $\gamma$ joining $s^{-1}(x)$ to $s^{-1}(y)$. Here, by a geodesic chain joining $p=(a, u)$ to $q=(b, v)$ in $I \times K$, we mean a countable collection $\gamma=\left\{\gamma_{\nu}: \nu \in \mathbb{Z}\right\}$ of geodesic paths $\gamma_{\nu}:\left[a_{\nu}, b_{\nu}\right] \rightarrow I \times K$ satisfying the following conditions:

(i) $\gamma_{\nu}\left(b_{\nu}\right) \sim \gamma_{\nu+1}\left(a_{\nu+1}\right)$ for all $\nu \in \mathbb{Z}$, and

(ii) $\gamma_{\nu}\left(a_{\nu}\right) \rightarrow x$ as $\nu \rightarrow-\infty$ and $\gamma_{\nu}\left(b_{\nu}\right) \rightarrow y$ as $\nu \rightarrow \infty$.

Note that we allow some of the paths $\gamma_{\nu}$ to be trivial. The resulting metric space $(X, d)$ is a geodesic space. Moreover, the measure $\mu$ on $I \times K$ descends to a measure (still denoted $\mu$ ) on $X$ which is Ahlfors $Q$-regular [46. Section 1]; this fact is easily proved by noting that the quotient map $s$ is regular in the sense of David and Semmes [22, Definition 12.1] and using [22, Lemma 12.7]. By [46, Theorem 2.6], the metric measure space $(X, d, \mu)$ satisfies the Loewner condition.

\section{PART 3. Applications}

\section{Quasisymmetric inVARIANCE of the Loewner CONDItion AND OF Poincaré INEQUalities}

As mentioned in section 8 , Proposition 8.9 implies that almost every curve (in the sense of the generalized modulus) in a locally compact, locally Ahlfors $Q$-regular space, $Q>1$, is locally rectifiable. It follows that for quasisymmetric maps between domains in such spaces, the collection of locally rectifiable curves whose image is not locally rectifiable has generalized (and hence also classical) modulus equal to zero. Thus nontriviality of the classical modulus is a quasisymmetry invariant. A quantitative version of this fact follows from Corollary 6.7. For the corresponding result in globally Ahlfors regular spaces, see [69, Corollary 1.6].

Corollary 12.1. Let $X$ and $Y$ be locally compact, locally Ahlfors $Q$-regular metric spaces, $Q>1$, and let $f: X \rightarrow Y$ be an $\eta$-quasisymmetric homeomorphism. If $X$ is Loewner, then $Y$ is also.

Corollary 12.1 is quantitative in the usual sense: the Loewner data of $Y$ depends only on the Loewner data of $X$, the regularity data of $X$ and $Y$ and the distortion function $\eta$.

Proof. Let $f: X \rightarrow Y$ be $\eta$-quasisymmetric and let $E^{\prime}$ and $F^{\prime}$ be disjoint, nondegenerate continua in $Y$ satisfying

$$
\operatorname{dist}\left(E^{\prime}, F^{\prime}\right) \leq t \min \left\{\operatorname{diam} E^{\prime}, \operatorname{diam} F^{\prime}\right\}
$$


for some $t>0$. Then $f^{-1}$ is $\eta_{L}$-quasisymmetric, where $\eta_{L}(t)=\eta^{-1}\left(t^{-1}\right)^{-1}$ and so

$$
\operatorname{dist}(E, F) \leq \eta_{L}(2 t) \min \{\operatorname{diam} E, \operatorname{diam} F\} .
$$

Corollary 6.7 implies that $f$ is geometrically $K$-quasiconformal for some $K$ depending only on the regularity data of $X$ and $Y$. If $\phi:(0, \infty) \rightarrow(0, \infty)$ is a Loewner function for $X$, then the function $\psi:(0, \infty) \rightarrow(0, \infty)$ given by

$$
\psi(t)=K^{-1} \cdot \phi \circ \eta_{L}(2 t)
$$

is a Loewner function for $Y$.

The Loewner condition is closely related to certain inequalities of Poincaré type, as observed by Heinonen and Koskela 38.

Definition 12.2. Let $p \geq 1$. A metric measure space $(X, \mu)$ is said to support a (weak) p-Poincaré inequality if there exist constants $C, C_{0} \geq 1$ so that the following holds true for every ball $B$ in $X$ : for every continuous function $u$ defined on $C_{0} B$ satisfying $\int_{B} u d \mu=0$ and for every Borel function $\rho$ defined on $C_{0} B$ satisfying

$$
|u(\gamma(a))-u(\gamma(b))| \leq \int_{\gamma} \rho d s
$$

for each rectifiable curve $\gamma:[a, b] \rightarrow C_{0} B$, we have

$$
\frac{1}{\mu(B)} \int_{B}|u| d \mu \leq C(\operatorname{diam} B)\left(\frac{1}{\mu\left(C_{0} B\right)} \int_{C_{0} B} \rho^{p} d \mu\right)^{1 / p} .
$$

If $u$ is a Lipschitz function on $X=\mathbb{R}^{n}$, then $u$ is differentiable a.e. by Rademacher's theorem and the gradient $\nabla u$ may be redefined a.e. so that the pair $(u,|\nabla u|)$ satisfies (12.3) on every ball in $\mathbb{R}^{n}$. Moreover, $|\nabla u|$ is the minimal such function, i.e., if $\rho$ is any other Borel function satisfying (12.3) for every rectifiable curve $\gamma$, then $|\nabla u(x)| \leq \rho(x)$ for a.e. $x \in B$. In this case, the 1-Poincaré inequality holds.

By Hölder's inequality, condition (12.4) becomes weaker as $p$ increases, that is, if $X$ supports a $p$-Poincaré inequality, then it also satisfies a $q$-Poincaré inequality for each $q>p$. The converse need not hold; for each $1<p \leq n$, there exists an $n$-regular domain in $\mathbb{R}^{n}$ supporting a $p$-Poincaré inequality but not a $q$-Poincaré inequality for any $q<p$ [4].

Poincaré inequalities are related to the Loewner condition as follows: every proper and quasiconvex Ahlfors $Q$-regular space, $Q>1$, is Loewner if and only if it supports a $Q$-Poincaré inequality [38, Corollary 5.13] 13 Here a metric space is said to be proper if every closed ball is compact. In fact, quasiconvexity can be replaced by the qualitative condition that the space be path connected since every $Q$-regular Loewner space is quasiconvex and every proper, path connected, Ahlfors $Q$-regular space satisfying a Poincaré inequality is also quasiconvex [32].

By results of Koskela and MacManus [45], if a locally compact Ahlfors $Q$-regular space supports a $p$-Poincaré inequality for some $p<Q$, then every locally compact and Ahlfors $Q$-regular quasisymmetric image also supports a $p^{\prime}$-Poincaré inequality for some $p^{\prime} \in[p, Q)$. However, for each $p>2$, there exist compact 2-regular spaces supporting a $p$-Poincaré inequality which have locally compact 2-regular quasisymmetric images supporting no Poincaré inequality at all. By the comments

\footnotetext{
${ }^{13}$ Note that all of the examples described in section 11] in fact support the 1-Poincaré inequality; this is stronger than the statement that they satisfy the Loewner condition.
} 
given in the preceding paragraph, Corollary 12.1 extends the result of Koskela and MacManus for $p<Q$ to the boundary case $p=Q$ (see also p. 532 in [69]):

Corollary 12.5. Let $X$ and $Y$ be proper, path connected, Ahlfors $Q$-regular spaces, $Q>1$, which are quasisymmetrically equivalent. If $X$ supports a $Q$-Poincaré inequality, then $Y$ does also, quantitatively.

\section{SURJECTIVITY FOR LOCALLY QUASISYMMETRIC MAPPINGS}

For any $n \geq 1$, the $n$-regular Euclidean space $\mathbb{R}^{n}$ is a Loewner space with exponent $n$. While the quantitative form of this result is due to Gehring, Loewner [47. had established a qualitative version several years earlier. More precisely, he showed that for any domain $G$ in $\mathbb{R}^{n}$ whose boundary $\partial G$ consists of two components $E$ and $F$, the conformal modulus $\operatorname{Mod}_{n}(E, F ; G)$ is positive if and only if both $E$ and $F$ are nondegenerate. From this fact he deduced the following corollary: $\mathbb{R}^{n}$ is not quasiconformally equivalent to any proper subdomain if $n \geq 2$. We prove a theorem which extends this result to the abstract setting of Ahlfors regular Loewner spaces.

Theorem 13.1. Let $X$ be a proper, $Q$-regular Loewner space, $Q>1$. Let $f: X \rightarrow$ $X$ be a locally quasisymmetric embedding (i.e., $f$ is a homeomorphism onto its image). If $f X$ is an open set in $X$, then $f$ is surjective and globally quasisymmetric.

If $X$ is not proper or $f X$ is not open in $X$, the conclusion need not hold. For example, we may take $X$ to be the open (resp. closed) upper halfspace in $\mathbb{C}$ and $f$ to be the map $f(z)=z+i$.

Note also that by Theorem 10.9 it suffices to assume only that $f$ satisfies (6.5).

Remark 13.2. Theorem 13.1 is similar to a result of Balogh and Koskela 3, Theorem 1.1] on the removability of porous sets for quasiconformal maps: if $X$ is a metric space satisfying the assumptions of 13.1 together with a certain separation axiom, then there exists $a \geq 1$ so that any quasiconformal embedding $f$ of the complement of a spherically $a$-porous set $E$ in $X$ onto an open set in $X$ which preserves boundedness extends to a quasisymmetric self-homeomorphism of $X$. (For the definition of porosity, see [3].) Theorem 13.1 (which corresponds to the choice $E=\emptyset$ ) differs from Theorem 1.1 of $[3$ in that no assumption comparable to the separation axiom is made. This axiom appears to be used in [3] both in defining the extension of $f$ to $E$ and also in verifying that the extended map is surjective and quasisymmetric. Although several examples are given in [3] showing the necessity of some type of separation condition, these examples are all related to the issue of constructing the extension and do not address the question of whether such a condition is necessary merely to prove surjectivity of an a priori globally defined map.

We turn now to the proof of Theorem 13.1. The basic framework is as follows. We first use Theorem 6.4 to deduce that the omitted set $X \backslash f X$ is sufficiently small. We then use this to promote the local quasisymmetry of $f$ to global quasisymmetry. Finally, we use our improved knowledge of $f$ to conclude that $X \backslash f X$ is in fact empty.

Note, however, that a priori we know only that the image set $f X$ is open. This implies that $f X$ is locally Ahlfors $Q$-regular and doubling and hence Theorem 6.4 can be applied to the homeomorphism $f: X \rightarrow f X$. This feature was a primary motivation for our use of the concept of local Ahlfors regularity in this paper. 
The following concept was introduced by Shanmugalingam 62] in connection with her study of Sobolev functions on arbitrary metric spaces.

Definition 13.3. Let $(X, \mu)$ be a rectifiably connected metric measure space and let $p \geq 1$. For $\rho \in L^{p}(X, \mu)$, define an equivalence relation $\stackrel{\rho}{\sim}$ on $X$ as follows:

$x \stackrel{\rho}{\sim} y$ if and only if either $x=y$ or there exists a curve $\gamma$ joining $x$ to $y$ with $\int_{\gamma} \rho d s<\infty 14$

We say that $X$ has a main equivalence class for exponent $p$ (for short, $X$ has the $\mathrm{MEC}_{p}$ condition) if for each $\rho \in L^{p}(X, \mu)$ there exists an equivalence class $G_{\rho} \subset X$ for which $\mu\left(X \backslash G_{\rho}\right)=0$.

Every $Q$-regular Loewner space has the $\mathrm{MEC}_{Q}$ condition. On the other hand, for any $p \geq 1$, every space satisfying the $\mathrm{MEC}_{p}$ condition for some nontrivial measure $\mu$ has nontrivial $p$-modulus, i.e., there exists a curve family $\Gamma$ in $X$ so that $\operatorname{Mod}_{p} \Gamma>0$. For proofs of these results, see 62]. (The proof of the first claim is similar to that of Lemma 14.2)

For $A, B \subset X$ we denote by $(A ; B)$ the collection of all curves $\gamma$ for which $\overline{|\gamma|} \cap A \neq \emptyset$ and $|\gamma| \subset B$.

Proof of Theorem 13.1. Let $X$ and $f$ satisfy the hypotheses and set $E^{\prime}=X \backslash f X$. Let

$$
\begin{aligned}
\Gamma & =f^{-1}\left(E^{\prime} ; f X\right) \\
& =\left\{f^{-1} \circ \gamma: I \rightarrow X \mid \gamma: I \rightarrow Y \text { satisfies } \overline{|\gamma|} \cap E^{\prime} \neq \emptyset \text { and }|\gamma| \subset f X\right\}
\end{aligned}
$$

Since $X$ is proper, each curve in $\Gamma$ leaves every compact subset of $X$. It follows that $\operatorname{Mod}_{Q} \Gamma=0$. To see this, fix a basepoint $x_{0} \in X$ and denote by $K_{R}$ the compact ball $\bar{B}\left(x_{0}, R\right)$. For $n \in \mathbb{N}$, let $\Gamma_{n}$ denote the collection of curves $\gamma \in \Gamma$ for which $|\gamma|$ meets $K_{n}$. Then each curve in $\Gamma_{n}$ meets the complement of $K_{R}$ for every $R>n$, which implies that $\Gamma_{n}$ is minorized by the curve family $\left(K_{n}, X \backslash K_{R} ; \Omega_{R}\right)$, where $\Omega_{R}=B\left(x_{0}, R\right) \backslash \bar{B}\left(x_{0}, n\right)$. By (10.6), we conclude that

$$
\operatorname{Mod}_{Q} \Gamma_{n} \leq \lim _{R \rightarrow \infty} \operatorname{Mod}_{Q}\left(K_{n}, X \backslash K_{R} ; \Omega_{R}\right) \leq C \lim _{R \rightarrow \infty}(\log R / n)^{1-Q}=0
$$

and so $\Gamma=\bigcup_{n=1}^{\infty} \Gamma_{n}$ has $\operatorname{Mod}_{Q} \Gamma=0$. By Corollary $6.715 \operatorname{Mod}_{Q}\left(E^{\prime} ; f X\right)=0$.

We now show that $\mu\left(E^{\prime}\right)=0$. Since the curve family $\left(E^{\prime} ; f X\right)$ has $Q$-modulus zero, we may choose $\rho \in L^{Q}(X, \mu)$ so that $\int_{\gamma} \rho d s=\infty$ for each $\gamma \in\left(E^{\prime} ; f X\right)$ (see Proposition 1.4(v)). Since $X$ is Loewner, it satisfies the $\mathrm{MEC}_{Q}$ condition; let $G_{\rho}$ denote the main equivalence class for $\rho$. If $\mu\left(E^{\prime}\right)>0$, choose $x \in E^{\prime} \cap G_{\rho}$. Since $f X$ is open in $X$, it has positive measure and hence meets $G_{\rho}$. Let $y \in f X \cap G_{\rho}$ and let $\gamma$ be a rectifiable curve joining $x$ to $y$ with $\int_{\gamma} \rho d s<\infty$. Then $\gamma$ contains a subcurve $\gamma_{0} \in\left(E^{\prime} ; f X\right)$, but then $\int_{\gamma_{0}} \rho d s<\infty$ which contradicts the choice of $\rho$.

Next, we show that $\operatorname{Mod}_{Q}\left(E^{\prime} ; X\right)=0$. Let $\rho^{\prime}: X \rightarrow[0, \infty]$ denote the function which assumes the value $+\infty$ on $E^{\prime}$ and is zero elsewhere, so $\rho+\rho^{\prime} \in L^{Q}(X, \mu)$. Let $\gamma \in\left(E^{\prime} ; X\right)$. If $|\gamma| \subset E^{\prime}$ then $\int_{\gamma}\left(\rho+\rho^{\prime}\right) d s \geq \int_{\gamma} \rho^{\prime} d s=\infty$, while if $|\gamma| \cap f X \neq \emptyset$

\footnotetext{
${ }^{14}$ Note that we must include the option $x=y$ explicitly since we restrict ourselves to nonconstant curves.

${ }^{15}$ This is the only point in the proof where the results of sections 66 10] are used.
} 
then $\gamma$ contains a subcurve $\gamma_{0} \in\left(E^{\prime} ; f X\right)$ and so $\int_{\gamma}\left(\rho+\rho^{\prime}\right) d s \geq \int_{\gamma_{0}} \rho d s=\infty$. Thus $\operatorname{Mod}_{Q}\left(E^{\prime} ; X\right)=0$ and hence $E^{\prime}$ is removable for the conformal modulus in $X$ :

$$
\operatorname{Mod}_{Q} \Gamma=\operatorname{Mod}_{Q}\left\{\gamma \in \Gamma: \overline{|\gamma|} \subset X \backslash E^{\prime}=f X\right\}, \quad \Gamma \text { a curve family in } X .
$$

(Note here also that $f X$ is dense in $X$.) We deduce that $X \backslash E^{\prime}=f X$ is also a Loewner space. Moreover, since $\mu\left(E^{\prime}\right)=0, f X$ is also Ahlfors $Q$-regular.

Now $f: X \rightarrow f X$ is a locally quasisymmetric homeomorphism between $Q$ regular Loewner spaces. Since $X$ is proper, $f$ takes bounded sets to bounded sets. By a result of Heinonen and Koskela [38, Corollary 4.10], $f$ is globally quasisymmetric. From this it easily follows that $E^{\prime}$ is empty: if $x^{\prime} \in X$, choose $x_{n}^{\prime} \in f X$ with $x_{n}^{\prime} \rightarrow x^{\prime}$ and let $x_{n}=f^{-1} x_{n}^{\prime}$. Since $\left\{x_{n}^{\prime}\right\}$ is a Cauchy sequence, so is $\left\{x_{n}\right\}$ [68, Theorem 2.24]. Thus $\left\{x_{n}\right\}$ converges to some point $x \in X$, whence $x^{\prime}=f x \in f X$.

\section{Absolute continuity}

In this section, we study the absolute continuity of locally quasisymmetric maps between locally Ahlfors $Q$-regular spaces, $Q>1$.

Recall that a mapping $f:(X, \mu) \rightarrow(Y, \nu)$ between measure spaces is called $a b$ solutely continuous in measure or equivalently, is said to satisfy the Lusin condition $(N)$, if every set in $X$ of $\mu$-measure zero is taken to a set in $Y$ of $\nu$-measure zero.

When the spaces $(X, \mu)$ and $(Y, \nu)$ are locally Ahlfors $Q$-regular metric measure spaces, we say that $f: X \rightarrow Y$ is absolutely continuous along almost every curve if the collection of locally rectifiable curves $\gamma: I \rightarrow X$ for which the curve $f \circ \gamma: I \rightarrow Y$ is not absolutely continuous has $Q$-modulus zero. Recall that a locally rectifiable curve $\gamma: I \rightarrow X$ is called absolutely continuous if $\int_{\gamma} \chi_{E} d s=0$ for every Borel set $E \subset I$ with $\mathcal{L}_{1}(E)=0$. (Here $\mathcal{L}_{1}$ denotes Lebesgue measure on the interval $I$.)

By a general result of Heinonen, Koskela, Shanmugalingam, and the author [39. Theorem 9.8], quasisymmetric maps between locally compact $Q$-regular Loewner spaces are absolutely continuous in measure and along $Q$-almost every curve. This result is established in two parts: first, such maps are shown to be metric spacevalued Sobolev mappings in the sense of Reshetnyak (see the historical remark below) which fact immediately yields the absolute continuity along curves, and second, the absolute continuity along curves is used to derive absolute continuity in measure. In this section, we briefly sketch this latter half of the argument, which makes use of Theorem 6.4 Specifically, we will prove the following theorem.

Theorem 14.1. Let $f: U \rightarrow U^{\prime}$ be a locally quasisymmetric homeomorphism between open sets $U \subset X$ and $U^{\prime} \subset Y$ contained in locally compact, Ahlfors $Q$ regular Loewner spaces, $Q>1$. If $f$ is absolutely continuous along $Q$-a.e. curve, then $f$ is absolutely continuous in measure.

Fix now a metric measure space $(X, \mu)$ and an open set $U \subset X$. For each Borel set $A \subset U$, let $\Gamma_{A}^{+}$denote the collection of locally rectifiable curves $\gamma: I \rightarrow U$ for which $\int_{\gamma} \chi_{A} d s>0$. Note that if $\mu(A)=0$, then the function $\rho: U \rightarrow[0, \infty]$ given by $\rho(x)=\infty$ if $x \in A$ and $\rho(x)=0$ otherwise is in $L^{Q}(U, \mu)$ and satisfies $\int_{\gamma} \rho d s=\infty \geq 1$ for all $\gamma \in \Gamma_{A}^{+}$. Thus $\operatorname{Mod}_{Q} \Gamma_{A}^{+}=0$. In the particular case of $Q$-regular Loewner spaces, the converse also holds:

Lemma 14.2. Let $A$ be a Borel subset of an open set $U$ in a $Q$-regular Loewner space $(X, \mu)$. If $\operatorname{Mod}_{Q} \Gamma_{A}^{+}=0$, then $\mu(A)=0$. 
For a proof, combine Theorem 6.2 and Lemma 6.5 of 62 .

Proof of Theorem 14.1. For the proof that $f$ is absolutely continuous along $Q$-a.e. curve, we refer the reader to [39, Section 8]. Suppose now that $f$ is absolutely continuous along $Q$-a.e. curve and assume that $E \subset X$ has $\mu(E)=0$ but $\nu(f E)>0$. By Lemma 14.2, $\operatorname{Mod}_{Q} \Gamma_{E}^{+}=0$ but $\operatorname{Mod}_{Q} \Gamma_{f E}^{+}>0$. By Corollary6.7, together with the fact that $Q$-almost every locally rectifiable curve in $X$ is taken onto a locally rectifiable curve in $Y$, it suffices to prove that

$$
f^{-1} \Gamma_{f E}^{+} \subset \Gamma_{E}^{+} \cup \Gamma_{N R} \cup \Gamma_{N A C},
$$

where $\Gamma_{N R}$ denotes the collection of nonlocally rectifiable curves in $X$ and $\Gamma_{N A C}$ denotes the collection of locally rectifiable curves $\gamma$ in $X$ along which $f \circ \gamma$ is not absolutely continuous.

Thus, suppose that (14.3) fails to hold. Then there exists a locally rectifiable curve $\gamma: I \rightarrow X$ for which $f \circ \gamma$ is absolutely continuous and $\int_{\gamma} \chi_{E} d s=0$ but $\int_{f \circ \gamma} \chi_{f E} d s>0$. In this case $E_{0}=\gamma^{-1}(E)$ is a set in $I$ with $\mathcal{L}_{1}\left(E_{0}\right)=0$ but $\int_{f \circ \gamma} \chi_{(f \circ \gamma)\left(E_{0}\right)} d s>0$, which contradicts the absolute continuity of $f \circ \gamma$.

Historical Remarks 14.4. The absolute continuity in measure of quasiconformal maps between domains in $\mathbb{R}^{n}, n \geq 2$, is due to Gehring [29]. Absolute continuity along $n$-a.e. curve follows from a theorem of Fuglede [27], since quasiconformal maps of domains in $\mathbb{R}^{n}$ lie in the local Sobolev space $W_{\text {loc }}^{1, n}$.

These results were extended by Väisälä 72 to locally quasisymmetric maps from a domain $\Omega \subset \mathbb{R}^{n}$ to any set $Y \subset \mathbb{R}^{N}, N<\infty$, with locally finite Hausdorff $n$-measure and then by the author [70] to the case of general metric spaces $Y$ with locally finite Hausdorff $n$-measure. Here the natural analog of the absolute continuity in measure is that sets of zero (Lebesgue) $n$-measure in $\Omega$ are taken to sets of zero Hausdorff $n$-measure in $Y$, while an appropriate definition for the Sobolev class $W_{\text {loc }}^{1, n}$ of mappings from $\Omega$ into an abstract metric space was given by Reshetnyak [57]. Note that Ahlfors $n$-regularity of the target space is not assumed in these results.

Heinonen and Koskela 38. Sections 7 and 8] established the absolute continuity in measure and along $Q$-a.e. curve for quasisymmetric maps in Ahlfors $Q$-regular spaces, $Q>1$, satisfying a $p$-Poincaré inequality for some $1 \leq p<Q 16$ All of the preceding results are subsumed in the general result of [39] mentioned above.

\section{Conformal AND Metric GeOMetry of Products OF SNOWFLAKE CURVES}

Definition 15.1. Let $X=(X, d)$ be a metric space and let $0<\epsilon \leq 1$. Define $d_{\epsilon}: X \times X \rightarrow \mathbb{R}$ by

$$
d_{\epsilon}(x, y)=d(x, y)^{\epsilon}, \quad x, y \in X .
$$

It is easily verified that $d_{\epsilon}$ is in fact a metric on $X$. We call the metric space $\left(X, d_{\epsilon}\right)$ the snowflake of $X$ (of order $\epsilon$ ).

\footnotetext{
${ }^{16}$ In fact, they show that the pullback of the $Q$-regular measure in the image is $A_{\infty}$-related to the $Q$-regular measure in the source; this extends the celebrated higher integrability theorem of Gehring [30].
} 
Note that the identity map from $X$ to $\left(X, d_{\epsilon}\right)$ is quasisymmetric with distortion function $\eta(t)=t^{\epsilon}$. Moreover, the dimension of $\left(X, d_{\epsilon}\right)$ is equal to $\operatorname{dim} X / \epsilon$, and whenever $X$ is Ahlfors regular, so is $\left(X, d_{\epsilon}\right)$. The metric space $\left(X, d_{\epsilon}\right)$ carries no nontrivial locally rectifiable curves if $\epsilon<1$.

If $d$ denotes the standard Euclidean metric on $\mathbb{R}$ and $\epsilon=\log 3 / \log 4$, then $\left(\mathbb{R}, d_{\epsilon}\right)$ is bi-Lipschitz equivalent to the classical von Koch snowflake curve $C$ (Figure 5).

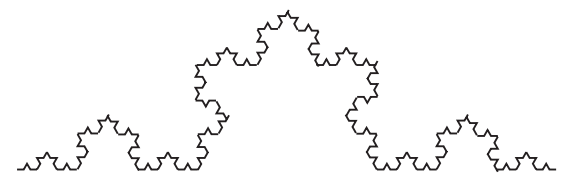

Figure 5. The von Koch snowflake curve

For $n \geq 1$ and $\epsilon_{1}, \ldots, \epsilon_{n}$ in $(0,1]$, let $X(\bar{\epsilon}), \bar{\epsilon}=\left(\epsilon_{1}, \ldots, \epsilon_{n}\right)$, be the product of the $n$ spaces $\left(\mathbb{R}, d_{\epsilon_{i}}\right)$. Specifically, we let $X(\bar{\epsilon})$ be the space $\mathbb{R}^{n}$ endowed with the metric

$$
d_{\bar{\epsilon}}(x, y)=\max _{i=1}^{n}\left|x_{i}-y_{i}\right|^{\epsilon_{i}}, \quad x=\left(x_{1}, \ldots, x_{n}\right), y=\left(y_{1}, \ldots, y_{n}\right) \in \mathbb{R}^{n} .
$$

Then $X(\bar{\epsilon})$ has topological dimension $n$ (indeed, $X(\bar{\epsilon})$ is homeomorphic to $\mathbb{R}^{n}$ ) and is an Ahlfors $Q_{\bar{\epsilon}}$ regular space with

$$
Q_{\bar{\epsilon}}=\sum_{i=1}^{n} \frac{1}{\epsilon_{i}} .
$$

Theorem 15.3. Let $\bar{\epsilon}=\left(\epsilon_{1}, \ldots, \epsilon_{n}\right)$ and $\bar{\delta}=\left(\delta_{1}, \ldots, \delta_{n}\right)$ be as above. Then

(i) $X(\bar{\epsilon})$ and $X(\bar{\delta})$ are quasisymmetrically equivalent if and only if there exists a constant $\lambda>0$ and $\sigma \in S_{n}$ so that $\epsilon_{i}=\lambda \delta_{\sigma(i)}$ for all $i=1, \ldots, n$;

(ii) $X(\bar{\epsilon})$ and $X(\bar{\delta})$ are bi-Lipschitz equivalent if and only if there exists $\sigma \in S_{n}$ so that $\epsilon_{i}=\delta_{\sigma(i)}$ for all $i=1, \ldots, n$.

Here $S_{n}$ denotes the symmetric group on $n$ letters, i.e., the collection of all permutations of the $n$-element set $\{1, \ldots, n\}$.

Theorem 15.3 can be informally restated as follows: $X(\bar{\epsilon})$ and $X(\bar{\delta})$ are biLipschitz equivalent if and only if they are isometric and $X(\bar{\epsilon})$ and $X(\bar{\delta})$ are quasisymmetrically equivalent if and only if they are conformally equivalent.

Theorem 15.3 also admits a compact version, where the space $\prod_{i=1}^{n}\left(\mathbb{R}, d_{\epsilon_{i}}\right)$ is replaced by $\prod_{i=1}^{n}\left([0,1], d_{\epsilon_{i}}\right)$. The proof is completely analogous.

Example 15.4. Let $n=2$ and let $\epsilon_{1}=1$ and $\epsilon_{2}=\log 3 / \log 4$. The space $X(\bar{\epsilon})$ is bi-Lipschitz equivalent to Rickman's rug, the product of the real line $\mathbb{R}$ with the von Koch snowflake curve $C$. The fact that the surface $\mathbb{R} \times C$ is not quasisymmetrically equivalent to $\mathbb{R}^{2}$ was established by Rickman (unpublished). More generally, Tukia showed in [67, Theorem 5] that $\mathbb{R}^{n-1} \times C$ is not quasisymmetrically equivalent to $\mathbb{R}^{n}$ for any $n \geq 2$. Both of these results are special cases of Theorem 15.3

Remark 15.5. Alestalo and Väisälä [1] give necessary conditions for the existence of a quasisymmetric embedding

$$
f: \prod_{i=1}^{n}\left([0,1]^{p_{i}}, d_{i}\right) \hookrightarrow \mathbb{R}^{p_{1}+\cdots+p_{n}},
$$


where $d_{i}$ is any metric on the $p_{i}$-dimensional unit cube $[0,1]^{p_{i}}$ which induces the Euclidean topology.

The reverse implications in Theorem 15.3 are immediate, so we focus our attention on the forward direction in each statement. Notice that (ii) follows immediately once we have established (i). Indeed, suppose that $X(\bar{\epsilon})$ and $X(\bar{\delta})$ are bi-Lipschitz equivalent. Then they are also quasisymmetrically equivalent and so there exists $\lambda>0$ and $\sigma \in S_{n}$ so that $\epsilon_{i}=\lambda \delta_{\sigma(i)}$ for each $i=1, \ldots, n$. Since bi-Lipschitz maps preserve the Hausdorff dimension, we have

$$
\sum_{i=1}^{n} \frac{1}{\epsilon_{i}}=Q_{\bar{\epsilon}}=Q_{\bar{\delta}}=\sum_{i=1}^{n} \frac{1}{\delta_{i}}=\lambda \sum_{i=1}^{n} \frac{1}{\epsilon_{i}} .
$$

Hence $\lambda=1$ and the proof of (ii) is complete.

Before beginning the proof of part (i), we state a few brief remarks. Recall that a metric measure space $X=(X, d, \mu)$ is said to have nontrivial $p$-modulus, $p \geq 1$, if the $p$-modulus of the collection of all curves in $X$ is positive.

Remark 15.6. Ahlfors $Q$-regular metric spaces with nontrivial $Q$-modulus have minimal dimension among all Ahlfors regular quasisymmetric images. (In particular, this is true for Ahlfors regular Loewner spaces.) This follows from the fact that snowflaked metric spaces have trivial $p$-modulus for all $p$ (since they contain no nontrivial locally rectifiable curves). Indeed, suppose that $X$ is an Ahlfors $Q$-regular metric space with nontrivial $Q$-modulus and suppose that $Y$ is an Ahlfors $Q^{\prime}$-regular space quasisymmetrically equivalent to $X$ with $Q^{\prime}<Q$. Then the snowflake of $Y$ of order $\epsilon=Q^{\prime} / Q<1$ would be an Ahlfors $Q$-regular space with trivial $Q$-modulus, contradicting Corollary 6.7 .

The preceding result holds more generally without the assumption of Ahlfors regularity in the target: any metric measure space which satisfies the upper mass bound (1.2) with exponent $Q$ and has nontrivial $Q$-modulus has minimal dimension among all quasisymmetric images (see [7, Section 5, Remark 1]).

On the other hand, any Ahlfors regular metric space which contains a Euclidean factor has nontrivial conformal modulus.

Example 15.7. Let $Q>1$. Let $Z$ be any compact Ahlfors $(Q-1)$-regular metric space and let $X$ be the product space $Z \times[0,1]$ (when $Z$ is unbounded we use instead $X=Z \times \mathbb{R}$ ). Then $X$ is a compact $Q$-regular space and the family of curves

$$
\Gamma=\left\{\gamma_{z}:[0,1] \rightarrow X \mid \gamma_{z}(t)=(z, t), z \in Z\right\}
$$

has positive $Q$-modulus. This can be proved by a standard argument involving Fubini's theorem and Hölder's inequality (see e.g. Example 7.2 of [71]).

The proof of Theorem 15.3 will make use of the following lemma.

Lemma 15.8. Let $A \subset \mathbb{R}^{k}$ be a set which contains a.e. line parallel to each of the coordinate axes. Then $A$ contains a rectilinearly connected subset $A_{1}$ of full measure in $\mathbb{R}^{k}$.

Here we say that a set $A \subset \mathbb{R}^{k}$ is rectilinearly connected if any two points $x, y \in A$ can be joined by a curve $\gamma,|\gamma| \subset A$, consisting of the union of a number of line segments parallel to the coordinate axes. 
In what follows, we use the standard Lebesgue measure on $\mathbb{R}^{k}$ and statements referring to "almost every line parallel to a coordinate axis" in $\mathbb{R}^{k}$ refer to the Lebesgue $(k-1)$-measure on the orthogonal space to this family of lines; in the case $k=1$ we use the trivial measure on the one-point set.

Proof of Lemma 15.8. We induct on the dimension $k$. The case $k=1$ is trivial. Suppose that the lemma holds for some dimension $k$ and let $A \subset \mathbb{R}^{k+1}$ be a set containing a.e. line parallel to each of the coordinate axes in $\mathbb{R}^{k+1}$. Then there exists a set $B \subset \mathbb{R}^{k}$ of full measure so that $\{b\} \times \mathbb{R} \subset A$ for each $b \in B$. Also, for a.e. $t \in \mathbb{R}$, there exists a set $A^{\prime}(t) \subset \mathbb{R}^{k}$ of full measure so that $A^{\prime}(t) \times\{t\} \subset A$ and $A^{\prime}(t)$ contains a.e. line parallel to each of the coordinate axes in $\mathbb{R}^{k}$ (this follows by a standard application of Fubini's theorem). By the induction hypothesis, $A^{\prime}(t)$ contains a rectilinearly connected subset $A_{1}^{\prime}(t)$ of full measure in $\mathbb{R}^{k}$. Let $Z \subset \mathbb{R}$ be a countable dense subset of the set of $t^{\prime}$ 's for which $A^{\prime}(t)$ exists. Then $B_{1}:=B \cap \bigcap_{t \in Z} A^{\prime}(t)$ is of full measure in $\mathbb{R}^{k}$. Set

$$
A_{1}=\left(\bigcup_{t \in Z} A^{\prime}(t) \times\{t\}\right) \cup\left(\bigcup_{b \in B_{1}}\{b\} \times \mathbb{R}\right) .
$$

Then $A_{1} \subset A$ and $A_{1}$ is a rectilinearly connected set of full measure in $\mathbb{R}^{k}$, which completes the proof.

Proof of Theorem $15.3(i)$. Let $X(\bar{\epsilon})$ and $X(\bar{\delta})$ be quasisymmetrically equivalent. By permuting the coordinates if necessary, we may assume that

$$
\epsilon_{1} \geq \epsilon_{2} \geq \cdots \geq \epsilon_{n}
$$

and

$$
\delta_{1} \geq \delta_{2} \geq \cdots \geq \delta_{n}
$$

Moreover, we may also assume that $\epsilon_{1}=\delta_{1}=1$ since $X(\bar{\epsilon})$ is quasisymmetrically equivalent to $X\left(\bar{\epsilon}^{\prime}\right)$, where $\bar{\epsilon}^{\prime}=\left(1, \epsilon_{2} / \epsilon_{1}, \ldots, \epsilon_{n} / \epsilon_{1}\right)$.

For ease of notation, write $X=X(\bar{\epsilon})$ and $Y=X(\bar{\delta})$. Let $k$ be the number of exponents $\epsilon_{i}$ which equal 1 and let $l$ be the corresponding quantity for the $\delta_{i}$. We decompose $X=\mathbb{R}^{k} \times X_{1}$ and $Y=\mathbb{R}^{l} \times Y_{1}$. For $a \in X_{1}$, we call the subspace $\mathbb{R}^{k} \times\{a\}$ the "fiber over $a$ " and similarly for $b \in Y_{1}$. Since $k \geq 1$ and $l \geq 1$, Example 15.7 shows that $X$ has nontrivial $Q_{\bar{\epsilon}}$-modulus and that $Y$ has nontrivial $Q_{\bar{\delta}}$-modulus. By Remark 15.6] $Q_{\bar{\epsilon}}=Q_{\bar{\delta}}$. Call this common value $Q$.

Let $f: \mathbb{R}^{k} \times X_{1} \rightarrow \mathbb{R}^{l} \times Y_{1}$ be a quasisymmetric homeomorphism. The remainder of the proof will be devoted to showing that $k=l$ and that the map $f$ induces a quasisymmetric map $h: X_{1} \rightarrow Y_{1}$. By repeating this argument for $h$ and iterating, we will find that the sequences of exponents $\bar{\epsilon}=\left(\epsilon_{1}, \ldots, \epsilon_{n}\right)$ and $\bar{\delta}=\left(\delta_{1}, \ldots, \delta_{n}\right)$ are the same, which is the desired conclusion.

Recall from section 12 that $\operatorname{Mod}_{Q}$-a.e. locally rectifiable curve in $X$ is taken by $f$ to a locally rectifiable curve in $Y$. Note that a curve in $X$ (or $Y$ ) is locally rectifiable only when its projection into the second factor $X_{1}$ (or $Y_{1}$ ) is constant (since $X_{1}$ and $Y_{1}$ are snowflaked spaces).

Consider the family of lines parallel to the $x_{1}$-axis in $X$, where $x_{1}$ denotes the first coordinate of $\mathbb{R}^{k}$. Since all of these lines are locally rectifiable, the usual argument involving Fubini's theorem implies that there exists a subset $E_{1} \subset X_{1}$ of full measure so that for each $a \in E_{1}, f$ takes almost every line parallel to the $x_{1}$-axis in the fiber over $a$ to a locally rectifiable curve in $Y$. By repeating this 
procedure $k$ times, we find a subset $E \subset X_{1}$ of full measure so that for each $a \in E$, $f$ takes almost every line parallel to any coordinate axis in the fiber over $a$ onto a locally rectifiable curve in $Y$. Note that each of these curves must lie within a fiber in $Y$ by earlier remarks. By Lemma [15.8, the union of this collection of lines contains a rectilinearly connected set of full measure in $\mathbb{R}^{k}$, and hence the images of all of these lines must lie within the same fiber in $Y$ (which must necessarily be the fiber over $b$, where $b$ denotes the $Y_{1}$-coordinate of the point $\left.f(0, a)\right)$. But now continuity implies that the entire fiber over $a$ (a copy of $\mathbb{R}^{k}$ ) is taken into the fiber over $b$ (a copy of $\mathbb{R}^{l}$ ). Hence $k \leq l$. By following the same line of reasoning for the inverse map $f^{-1}$, we see that $k=l$.

The above results held only for almost every point in $X_{1}$ and we now wish to extend them to every point in $X_{1}$. For an arbitrary point $a \in X_{1}$, choose a sequence $a_{n} \in E$ converging to $a$. Then the entire fiber over $a_{n}$ is taken into the fiber over $b_{n}$ (notation as above). If $x$ is an arbitrary point in the fiber over $a$, then we can find a sequence of points in the fibers over $a_{n}$ converging to $x$. As these points are taken into the fibers over $b_{n}$, we conclude that $x$ is taken into the fiber over $b$.

We have shown that for each point $a \in X_{1}$, the entire fiber over $a$ is taken onto the fiber over the $Y_{1}$-coordinate of $f(0, a)$. Put another way, the map $f: X \rightarrow Y$ can be written as

$$
f(p, a)=(g(p, a), h(a)) \in \mathbb{R}^{k} \times Y_{1},
$$

where $p \in \mathbb{R}^{k}$ and $a \in X_{1}$. The maps $g: X \rightarrow \mathbb{R}^{k}$ and $h: X_{1} \rightarrow Y_{1}$ are both continuous. In addition, for fixed $a \in X_{1}$, the map $g_{a}: \mathbb{R}^{k} \rightarrow \mathbb{R}^{k}$ given by $g_{a}(p)=$ $g(p, a)$ is quasisymmetric. Since we have a similar decomposition for the inverse map $f^{-1}: Y \rightarrow X$, we see that $h$ is in fact a homeomorphism of $X_{1}$ onto $Y_{1}$.

We prove now that $h$ is quasisymmetric. The proof relies on the following lemma:

Lemma 15.9. Let $f: X \rightarrow Y$ be an $\eta$-quasisymmetric homeomorphism of metric spaces and let $A, B, C \subset X$. If $\operatorname{dist}(A, B) \leq t \operatorname{dist}(A, C)$ for some $t \geq 0$, then there exists $a \in A$ so that

$$
\operatorname{dist}(f A, f B) \leq \eta(t) \operatorname{dist}(f(a), f C) .
$$

We defer the proof of this lemma momentarily. If $A$ denotes the fiber in $X$ over the point $a$ and $B$ denotes the fiber in $Y$ over $b$, then $|a-b|=\operatorname{dist}(A, B)$. Let $a, b, c \in X_{1}$ and let $A, B, C$ be the fibers over $a, b, c$ respectively. If $|a-b| \leq t|a-c|$ for some $t \geq 0$, then $\operatorname{dist}(A, B) \leq t \operatorname{dist}(A, C)$ and so (by the lemma) there exists a point $(p, a) \in A$ so that

$$
|h(a)-h(b)|=\operatorname{dist}(f A, f B) \leq \eta(t) \operatorname{dist}(f(p, a), f C) .
$$

But $f(p, a)=(g(p, a), h(a))$ and $f C$ is the fiber over $h(c)$. It follows that

$$
\operatorname{dist}(f(p, a), f C)=|h(a)-h(c)|
$$

and so $h$ is quasisymmetric.

Since $X_{1}$ and $Y_{1}$ have strictly smaller dimension than $X$ and $Y$, we may (as mentioned above) iterate this procedure to reach the desired conclusion. This completes the proof of Theorem 15.3.

Proof of Lemma 15.9. Suppose that $\operatorname{dist}(A, B) \leq t \operatorname{dist}(A, C)$. If $\operatorname{dist}(A, C)=0$, then $\operatorname{dist}(A, B)=0$ and so $\bar{A}$ meets both $\bar{B}$ and $\bar{C}$. Since $f$ is a homeomorphism, $\overline{f A}$ meets both $\overline{f B}$ and $\overline{f C}$ and so $\operatorname{dist}(f A, f B)=\operatorname{dist}(f A, f C)=0$ and the proof is complete. 
Suppose now that $\operatorname{dist}(A, C)>0$. Then $\operatorname{dist}(A, B)<t^{\prime} \operatorname{dist}(A, C)$ for any $t^{\prime}>t$. Pick $a \in A$ and $b \in B$ so that $|a-b|<t^{\prime}\left|a^{\prime}-c\right|$ for any $a^{\prime} \in A$ and $c \in C$. Since this holds in particular for $a^{\prime}=a$, we conclude that $\operatorname{dist}(f A, f B) \leq|f(a)-f(b)| \leq$ $\eta\left(t^{\prime}\right)|f(a)-f(c)|$. Taking the infimum over all $c \in C$ establishes the claim.

As a corollary, the proof of Theorem 15.3 indicates the structure of any quasisymmetric map $f: X(\bar{\epsilon}) \rightarrow X(\bar{\delta})$. Assume that $\bar{\epsilon}$ and $\bar{\delta}$ satisfy $\epsilon_{i}=\lambda \delta_{\sigma(i)}$, $i=1, \ldots, n$, for some $\lambda>0$ and $\sigma \in S_{n}$ and assume that $\epsilon_{1} \geq \cdots \geq \epsilon_{n}$. Choose $1 \leq i_{1}<i_{2}<\cdots<i_{k-1}<i_{k}=n$ so that

$$
\epsilon_{1}=\cdots=\epsilon_{i_{1}}>\epsilon_{i_{1}+1}=\cdots=\epsilon_{i_{2}}>\cdots>\epsilon_{i_{k-1}+1}=\cdots=\epsilon_{n} .
$$

Up to bi-Lipschitz equivalence, we have the decompositions

$$
X(\bar{\epsilon})=X_{k} \times \cdots \times X_{1}
$$

where $X_{j}$ denotes the snowflake of $\mathbb{R}^{i_{j}-i_{j-1}}$ of order $\epsilon_{i_{j}}, i_{0}=0$, and

$$
X(\bar{\delta})=Y_{k} \times \cdots \times Y_{1} .
$$

Then the map $f$ admits the following "upper-triangular" decomposition:

$$
f\left(a_{k}, \ldots, a_{1}\right)=\left(g_{k}\left(a_{k}, \ldots, a_{1}\right), \ldots, g_{2}\left(a_{2}, a_{1}\right), g_{1}\left(a_{1}\right)\right),
$$

where $a_{j} \in X_{j}, j=1, \ldots, k$ and the maps $g_{j}: X_{j} \times \cdots \times X_{1} \rightarrow Y_{j}$ have the following property: for fixed $a_{j-1}, \ldots, a_{1}$ in $X_{j-1}, \ldots, X_{1}$ respectively, the map

$$
\left(g_{k}\right)_{a_{j-1}, \ldots, a_{1}}: X_{j} \rightarrow Y_{j}
$$

given by

$$
\left(g_{k}\right)_{a_{j-1}, \ldots, a_{1}}(p)=g_{k}\left(p, a_{j-1}, \ldots, a_{1}\right)
$$

is quasisymmetric. Note that $X_{j}$ and $Y_{j}$ are each snowflaked copies of the metric space $\mathbb{R}^{i_{j}-i_{j-1}}$ and thus (after conjugating with the snowflaking maps) the map $\left(g_{k}\right)_{a_{j-1}, \ldots, a_{1}}$ can be identified as a quasiconformal self-map of $\mathbb{R}^{i_{j}-i_{j-1}}$ endowed with the standard metric.

\section{ApPEndix}

This appendix contains a brief discussion of Lemma 8.5 and its proof. The argument, which uses Hölder's inequality and the boundedness of a maximal operator of Hardy-Littlewood type on the dual of $L^{p}(X, \mu)$, is well-known, see e.g. Lemma 4.2 of [8]. For this reason we do not repeat the actual argument. Rather we only describe here the specific maximal operator which is used in the proof.

For the reader's convenience, we recall the statement of the lemma.

Lemma 16.1. Let $X=(X, d, \mu)$ be a locally doubling metric measure space with doubling constant $C_{\mu}$. Let $t>1$ and let $1 \leq p<\infty$. Then there exists a $\mathcal{B}_{1}(X)$-full collection $\widehat{\mathcal{C}}(t)$ of balls, depending on $t$ and the doubling constant $C_{\mu}$, and there exists $A=A\left(t, p, C_{\mu}\right)$, so that

$$
\int_{X}\left(\sum_{i} a_{i} \chi_{t B_{i}}\right)^{p} d \mu \leq A \int_{X}\left(\sum_{i} a_{i} \chi_{B_{i}}\right)^{p} d \mu
$$

whenever $B_{1}, B_{2}, \ldots$ are elements of $\widehat{\mathcal{C}}(t)$ and $a_{1}, a_{2}, \ldots$ are nonnegative real numbers. 
Recall that $\mu$ is said to be locally doubling with doubling constant $C_{\mu}$ if the collection of closed balls $B$ for which $\mu(2 B) \leq C_{\mu} \mu(B)$ is a $\mathcal{B}_{1}(X)$-full collection.

Let $L_{\text {loc }}^{1}(X, \mu)$ denote the collection of functions $f$ for which $\int_{B}|f|^{p} d \mu<\infty$ for each ball $B \subset X$. For each $\mathcal{B}_{1}(X)$-full collection $\mathcal{C}$ of metric balls in $X$, we define a maximal operator $M_{\mathcal{C}}$ on $L_{\text {loc }}^{1}(X, \mu)$ as follows:

$$
M_{\mathcal{C}} f(x):=\sup _{B(x, r) \in \mathcal{C}} \frac{1}{\mu(B(x, r))} \int_{B(x, r)}|f| d \mu .
$$

When $\mathcal{C}=\mathcal{B}_{1}(X)$, this agrees with the usual definition of the (centered) HardyLittlewood maximal operator $M$; for any $\mathcal{C}$ and $f \in L_{\text {loc }}^{1}(X, \mu)$, we have $M_{\mathcal{C}} f \leq M f$.

The basic properties of the Hardy-Littlewood maximal operator on spaces of homogeneous type (i.e., metric spaces with a doubling measure) hold true for the operator $M_{\mathcal{C}}$ on spaces with a locally doubling measure. More precisely, there exists a $\mathcal{B}_{1}(X)$-full collection $\mathcal{C}$ (depending on the doubling constant $C_{\mu}$ ) so that

$$
M_{\mathcal{C}}: L^{1}(X, \mu) \rightarrow \text { weak }-L^{1}(X, \mu)
$$

and

$$
M_{\mathcal{C}}: L^{q}(X, \mu) \rightarrow L^{q}(X, \mu)
$$

for $1<q \leq \infty$. Here weak $-L^{1}(X, \mu)$ denotes the space of functions $f: X \rightarrow \mathbb{R}$ for which $\sup _{t>0} t \cdot \mu\{x \in X: f(x)>t\}$ is finite. In fact, for any choice of $C_{\mu}^{\prime} \geq C_{\mu}^{3}$, we may take $\mathcal{C}$ to be the collection of closed balls $B$ for which $\mu(5 B) \leq C_{\mu}^{\prime} \mu(B)$.

By making use of the Hardy-Littlewood-type maximal operator $M_{\mathcal{C}}$, Lemma 16.1 may be proved along standard lines. Let $t>1$ be as in the hypotheses and let $\widehat{\mathcal{C}}(t)$ denote the collection of closed balls $B$ in $X$ for which

$$
\mu(15 t B) \leq C_{\mu}^{5} t^{\log _{2} C_{\mu}} \mu(B) .
$$

Then $\widehat{\mathcal{C}}(t)$ is a $\mathcal{B}_{1}(X)$-full collection. For elements $B_{1}, B_{2}, \ldots$ in $\widehat{\mathcal{C}}(t)$ and nonnegative real numbers $a_{1}, a_{2}, \ldots$, write $f_{t}(x):=\sum_{i} a_{i} \chi_{t B_{i}}(x)$. Following the argument given in [8, Lemma 4.2], we estimate

$$
\left\|f_{t}\right\|_{p} \leq C \sup _{g} \int_{X} f_{1}(y)\left(M_{\mathcal{C}} g\right)(y) d \mu(y),
$$

where the supremum is taken over all functions $g \in L^{q}(X, \mu), q=p /(p-1)$, with $\|g\|_{q} \leq 1$ and $\mathcal{C}$ is defined as in the paragraph preceding this proof with $C_{\mu}^{\prime}=C_{\mu}^{5} t^{\log _{2}} C_{\mu}$. Hölder's inequality together with the boundedness of the operator $M_{\mathcal{C}}$ as a map from $L^{q}(X, \mu)$ to $L^{q}(X, \mu)$ implies that

$$
\left\|f_{t}\right\|_{p} \leq C \sup _{g}\left\|f_{1}\right\|_{p}\left\|M_{\mathcal{C}} g\right\|_{q} \leq C \sup _{g}\left\|f_{1}\right\|_{p}\|g\|_{q} \leq C\left\|f_{1}\right\|_{p} .
$$

The proof is complete.

\section{ACKNOWLeDGMents}

I would like to express my appreciation to my advisor, Juha Heinonen, for his interest in this work and for many helpful discussions. I am also grateful to Mario Bonk for numerous suggestions regarding the correct definitions for the generalized modulus and in particular for his observations which led to the definition of the generalized arc-length measure in section 5 Finally, I would like to thank the referee for many detailed and useful comments which greatly improved the text. 


\section{REFERENCES}

[1] P. Alestalo and J. Väisälä, Quasisymmetric embeddings of products of cells into the Euclidean space, Ann. Acad. Sci. Fenn. Ser. A I Math. 19 (1994), 375-392. MR 95h:30023

[2] Z. Balogh, Hausdorff dimension distortion of QC mappings on the Heisenberg group, J. Anal. Math. 83 (2001), 289-312.

[3] Z. Balogh and P. Koskela, Quasiconformality, quasisymmetry and removability in Loewner spaces, Duke Math. J. 101 (2000), no. 3, 554-577, with an appendix by J. Väisälä. MR 2001d:30029

[4] Z. Balogh and J. T. Tyson, Polar coordinates and regularity of quasiconformal mappings in Carnot groups, preprint, 2001.

[5] Z. M. Balogh, I. Holopainen, and J. T. Tyson, Singular solutions, homogeneous norms and quasiconformal mappings on Carnot groups, Preprint \#269, University of Helsinki, September 2000.

[6] N. Benakli, Polyèdres hyperboliques, passage du local au global, Thèse, Université Paris-Sud, 1992.

[7] C. J. Bishop and J. T. Tyson, Locally minimal sets for conformal dimension, Ann. Acad. Sci. Fenn. Ser. A I Math. 26 (2001), 361-373.

[8] B. Bojarski, Remarks on Sobolev imbedding inequalities, Complex analysis, Joensuu 1987, Lecture Notes in Mathematics, no. 1351, Springer-Verlag, Berlin, 1988, pp. 52-68. MR 90b:46068

[9] M. Bourdon, Immeubles hyperboliques, dimension conforme et rigidité de Mostow, Geom. Funct. Anal. 7 (1997), 245-268. MR 98c:20056

[10] M. Bourdon and H. Pajot, Poincaré inequalities and quasiconformal structure on the boundaries of some hyperbolic buildings, Proc. Amer. Math. Soc. 127 (1999), no. 8, 2315-2324. MR 99j:30024

[11] A. Bruckner, J. Bruckner, and B. Thomson, Real analysis, Prentice-Hall, N.J., 1997.

[12] J. W. Cannon, The combinatorial Riemann mapping theorem, Acta Math. 173 (1994), 155234. MR 95k:30046

[13] J. W. Cannon, W. J. Floyd, and W. R. Parry, Squaring rectangles: the finite Riemann mapping theorem, The mathematical legacy of Wilhelm Magnus: groups, geometry and special functions (Brooklyn, NY, 1992), Contemp. Math., no. 169, Amer. Math. Soc., Providence, RI, 1994, pp. 133-212. MR 95g:20045

[14] J. W. Cannon and E. L. Swenson, Recognizing constant curvature discrete groups in dimension 3, Trans. Amer. Math. Soc. 350 (1998), 809-849. MR 98i:57023

[15] L. Capogna, Regularity of quasilinear equations in the Heisenberg group, Comm. Pure Appl. Math. 50 (1997), no. 9, 867-889.

[16] _ Regularity for quasilinear equations and 1-quasiconformal maps in Carnot groups, Math. Ann. 313 (1999), no. 2, 263-295. MR 2000a:35027

[17] L. Capogna and P. Tang, Uniform domains and quasiconformal mappings on the Heisenberg group, Man. Math. 86 (1995), 267-281. MR 96f:30019

[18] C. Champetier, Propriétés statistiques des groupes de présentation finie, Adv. Math. 116 (1995), 197-262. MR 96m:20056

[19] J. Cheeger, Differentiability of Lipschitz functions on metric measure spaces, Geom. Funct. Anal. 9 (1999), 428-517. MR 2000g:53043

[20] W.-L. Chow, Über Systeme von linearen partiellen Differentialgleichungen erster Ordnung, Math. Ann. 117 (1939), 98-105. MR 1:313d

[21] M. Coornaert, T. Delzant, and A. Papadopoulos, Géométrie et théorie des groupes, Lecture Notes in Mathematics, no. 1441, Springer-Verlag, Berlin, 1990, Les groupes hyperboliques de Gromov. [Gromov hyperbolic groups], With an English summary. MR 92f:57003

[22] G. David and S. Semmes, Fractured fractals and broken dreams: self-similar geometry through metric and measure, Oxford Lecture Series in Mathematics and its Applications, vol. 7, Clarendon Press Oxford University Press, 1997. MR 99h:28018

[23] G. A. Edgar, Packing measure as a gauge variation, Proc. Amer. Math. Soc. 122 (1994), 167-174. MR 94k:28009

[24] _ Integral, probability, and fractal measures, Springer-Verlag, New York, 1998. MR 99c:28024

[25] _ Packing measure in general metric spaces, Real Anal. Exchange (to appear). 
[26] H. Federer, Geometric measure theory, Die Grundlehren der mathematischen Wissenschaften, vol. 153, Springer-Verlag, New York, 1969. MR 41:1976

[27] B. Fuglede, Extremal length and functional completion, Acta Math. 98 (1957), 171-219. MR 20:4187

[28] F. W. Gehring, Symmetrization of rings in space, Trans. Amer. Math. Soc. 101 (1961), 499-519. MR 24:A2677

[29] _ Rings and quasiconformal mappings in space, Trans. Amer. Math. Soc. 103 (1962), 353-393. MR 25:3166

[30] _ The $L^{p}$-integrability of the partial derivatives of a quasiconformal mapping, Acta Math. 130 (1973), 265-277. MR 53:5861

[31] — Quasiconformal mappings, pp. 213-268, Internat. Atomic Energy Agency, Vienna, 1976. MR 58:1144]

[32] P. Hajłasz and P. Koskela, Sobolev met Poincaré, Memoirs Amer. Math. Soc. 145 (2000), no. 688, 101 pp. MR 2000j:46063

[33] B. Hanson and J. Heinonen, An n-dimensional space that admits a Poincaré inequality but has no manifold points, Proc. Amer. Math. Soc. 128 (2000), no. 11, 3379-3390. MR 2001e:43004

[34] J. Heinonen, Calculus on Carnot groups, Fall School in Analysis (Jyväskylä, 1994), vol. 68, Ber. Univ. Jyväskylä Math. Inst., Jyväskylä, 1995, pp. 1-31. MR 96j:22015

[35] _ A capacity estimate on Carnot groups, Bull. Sci. Math. 119 (1995), 475-484. MR 96j:22011

[36] J. Heinonen and I. Holopainen, Quasiregular maps on Carnot groups, J. Geom. Anal. 7 (1997), no. 1, 109-148. MR 99i:30037

[37] J. Heinonen and P. Koskela, Definitions of quasiconformality, Invent. Math. 120 (1995), 61-79. MR 96e:30051

[38] _ Quasiconformal maps in metric spaces with controlled geometry, Acta Math. 181 (1998), 1-61. MR 99j:30025

[39] J. Heinonen, P. Koskela, N. Shanmugalingam, and J. T. Tyson, Sobolev classes of Banach space-valued functions and quasiconformal mappings, J. Anal. Math. 85 (2001), 87-139.

[40] J. Heinonen and S. Semmes, Thirty-three yes or no questions about mappings, measures, and metrics, Conform. Geom. Dyn. 1 (1997), 1-12. MR 99h:28012

[41] W. Hurewicz and H. Wallman, Dimension theory, Princeton Mathematical Series, vol. 4, Princeton University Press, Princeton, N. J., 1941. MR 3:312b

[42] A. Korányi and H. M. Reimann, Quasiconformal mappings on the Heisenberg group, Invent. Math. 80 (1985), 309-338. MR 86m:32035

[43] _ Foundations for the theory of quasiconformal mappings on the Heisenberg group, Adv. Math. 111 (1995), 1-87. MR 96c:30021

[44] P. Koskela, Removable sets for Sobolev spaces, Ark. Mat. 37 (1999), no. 2, 291-304. MR 2001g:46077

[45] P. Koskela and P. MacManus, Quasiconformal mappings and Sobolev spaces, Studia Math. 131 (1998), 1-17. MR 99e:46042

[46] T. Laakso, Ahlfors $Q$-regular spaces with arbitrary $Q$ admitting weak Poincaré inequalities, Geom. Funct. Anal. 10 (2000), no. 1, 111-123.

[47] C. Loewner, On the conformal capacity in space, J. Math. Mech. 8 (1959), 411-414. MR 21:3538

[48] G. A. Margulis and G. D. Mostow, The differential of a quasi-conformal mapping of a CarnotCarathéodory space, Geom. Funct. Anal. 5 (1995), no. 2, 402-433. MR 96m:53038

[49] P. Mattila, Geometry of sets and measures in Euclidean spaces, Cambridge Studies in Advanced Mathematics, vol. 44, Cambridge University Press, Cambridge, 1995. MR 96h:28006

[50] G. D. Mostow, Quasi-conformal mappings in n-space and the rigidity of hyperbolic space forms, Inst. Hautes Études Sci. Publ. Math. 34 (1968), 53-104. MR 38:4679

[51] P. Pansu, Dimension conforme et sphère à l'infini des variétés à courbure négative, Ann. Acad. Sci. Fenn. Ser. A I Math. 14 (1989), 177-212. MR 90k:53079

[52] — Métriques de Carnot-Carathéodory et quasiisométries des espaces symétriques de rang un, Ann. of Math. (2) 129 (1989), 1-60. MR 90e:53058

[53] P. K. Rashevsky, Any two points of a totally nonholonomic space may be connected by an admissible line, Uch. Zap. Ped. Inst. im. Liebknechta, Ser. Phys. Math. 2 (1938), 83-94, in Russian.

[54] H. M. Reimann, Rigidity of H-type groups, Math. Z. (to appear). 
[55] _ An estimate for pseudoconformal capacities on the sphere, Ann. Acad. Sci. Fenn. Ser. A I Math. 14 (1989), 315-324. MR 90m:32041

[56] H. M. Reimann and F. Ricci, The complexified Heisenberg group, Preprint, 2000.

[57] Yu. G. Reshetnyak, Sobolev classes of functions with values in a metric space, Sibirsk. Mat. Zh. 38 (1997), 657-675. MR 98h:46031

[58] C. A. Rogers, Hausdorff measures, Cambridge University Press, London, 1970. MR 43:7576

[59] W. Rudin, Real and complex analysis, third ed., McGraw-Hill, New York, 1987. MR 88k:00002

[60] X. Saint Raymond and C. Tricot, Packing regularity of sets in n-space, Math. Proc. Cambridge Philos. Soc. 103 (1988), 133-145. MR 88m:28002

[61] S. Semmes, Finding curves on general spaces through quantitative topology, with applications to Sobolev and Poincaré inequalities, Selecta Math. 2 (1996), 155-295. MR 97j:46033

[62] N. Shanmugalingam, Newtonian spaces: an extension of Sobolev spaces to metric measure spaces, Rev. Mat. Iberoamericana 16 (2000), no. 2, 243-279. CMP 2001:07

[63] P. Tang, Quasiconformal homeomorphisms on CR 3-manifolds with symmetries, Math. Z. 219 (1995), 49-69. MR 96i:32016

[64] S. J. Taylor and C. Tricot, The packing measure of rectifiable subsets of the plane, Math. Proc. Cambridge Philos. Soc. 99 (1986), 285-296. MR 87b:28008

[65] B. S. Thomson, Construction of measures in metric spaces, J. London Math. Soc. (2) 14 (1976), 21-24. MR 54:10537

[66] C. Tricot, Two definitions of fractional dimension, Math. Proc. Cambridge Philos. Soc. 91 (1982), 57-74. MR 84d:28013

[67] P. Tukia, A quasiconformal group not isomorphic to a Möbius group, Ann. Acad. Sci. Fenn. Ser. A I Math. 6 (1981), 149-160. MR 83b:30019

[68] P. Tukia and J. Väisälä, Quasisymmetric embeddings of metric spaces, Ann. Acad. Sci. Fenn. Ser. A I Math. 5 (1980), 97-114. MR 82g:30038

[69] J. T. Tyson, Quasiconformality and quasisymmetry in metric measure spaces, Ann. Acad. Sci. Fenn. Ser. A I Math. 23 (1998), 525-548. MR 99i:30038

[70] - Analytic properties of locally quasisymmetric mappings from Euclidean domains, Indiana Univ. Math. J. 49 (2000), no. 3, 995-1016. CMP 2001:06

[71] J. Väisälä, Lectures on n-dimensional quasiconformal mappings, Lecture Notes in Mathematics, no. 229, Springer-Verlag, Berlin, 1971. MR 56:12260

[72] _ Quasisymmetric embeddings in Euclidean spaces, Trans. Amer. Math. Soc. 264 (1981), 191-204. MR 82i:30031

[73] _ Quasiconformal maps of cylindrical domains, Acta Math. 162 (1989), 201-225. MR 90f:30034

[74] S. Willard, General topology, Addison-Wesley, Reading, MA, 1970. MR 41:9173

[75] W. P. Ziemer, Extremal length and p-capacity, Michigan Math. J. 16 (1969), 43-51. MR 40:346

Department of Mathematics, University of Michigan, Ann Arbor, Michigan 481091109

Current address: Department of Mathematics, State University of New York at Stony Brook, Stony Brook, New York 11794-3651

E-mail address: tyson@math.sunysb.edu 